University of Tennessee Health Science Center

UTHSC Digital Commons

\title{
$5-2014$
}

\section{Persistant GATA2 Expression Promotes Self-Renewal of Myeloid Progenitors and Blocks Lymphoid Differentiation.}

\author{
Satish Kumar Nandakumar \\ University of Tennessee Health Science Center
}

Follow this and additional works at: https://dc.uthsc.edu/dissertations

Part of the Cells Commons, and the Medical Sciences Commons

\section{Recommended Citation}

Nandakumar, Satish Kumar , "Persistant GATA2 Expression Promotes Self-Renewal of Myeloid Progenitors and Blocks Lymphoid Differentiation." (2014). Theses and Dissertations (ETD). Paper 178. http://dx.doi.org/10.21007/etd.cghs.2014.0222. 


\title{
Persistant GATA2 Expression Promotes Self-Renewal of Myeloid Progenitors and Blocks Lymphoid Differentiation.
}

\begin{abstract}
The transcription factor GATA2 is highly expressed in hematopoietic stem cells (HSCs) and is downregulated during differentiation. Overexpression of GATA2 is frequently observed in acute myeloid leukemia. In previous studies, enforced expression of GATA2 using a MSCV GATA2 retroviral vector blocked differentiation of HSC and progenitors without inducing leukemia. We hypothesized that a lower dose of GATA2 can relieve the HSC block and eventually transform myeloid progenitors into leukemia stem cells. To test this hypothesis we generated a MSCV GATA2-ERT vector in which nuclear concentration of GATA2 can be regulated by Tamoxifen (TAM). The GATA2-ERT protein was confirmed to leak into nucleus of transduced BM cells even in the absence of TAM. This low nuclear GATA2-ERT led to enhanced self-renewal of myeloid progenitors in vitro and was able to immortalize primary bone marrow (BM) cells into IL-3 dependent myeloid cell lines. Continuous GATA2-ERT expression was also required for the proliferation of these immortalized lines. Nmyc and HoxA9 mRNA were significantly higher in GATA2-ERT immortalized lines compared to control immortalized lines. Repression of either Nmyc or Hoxa9 in GATA2-ERT immortalized cells impaired their proliferation suggesting that they are potential GATA2 targets mediating this effect.

Myeloid expansion and a block in T cell and B cell lineage differentiation was observed in transplant recipients of GATA2-ERT expressing BM cells without TAM. The myeloid expansion occurs after the Granulocyte Monocyte Progenitor (GMP) and the lymphoid block occurs after the Common Lymphoid Progenitor (CLP) in transgenic mice. Finally using the C352P mutant we showed that myeloid expansion and $B$ lymphoid block require DNA binding activity of GATA2-ERT and were not due to the dominant negative effect of the cytosolic form of this protein.
\end{abstract}

We were the first to demonstrate that GATA2 overexpression confers increased selfrenewal of myeloid progenitors which correlates with increased GATA2 mRNA levels frequently observed in human AML patients. We believe that the lower dose of GATA2 is critical to this phenotype. Interestingly no myeloid leukemias were observed in both the transplant and transgenic animals.

\section{Document Type}

Dissertation

\section{Degree Name}

Doctor of Philosophy (PhD)

\section{Program}

Biomedical Sciences

\section{Research Advisor}

Derek A. Persons, MD, PhD

\section{Keywords}

GATA2, hematopoiesis, lymphoid, myeloid, transcription factor

\author{
Subject Categories \\ Cells | Medical Sciences | Medicine and Health Sciences
}


PERSISTANT GATA2 EXPRESSION PROMOTES SELF-RENEWAL OF MYELOID PROGENITORS AND BLOCKS LYMPHOID DIFFERENTIATION

\author{
A Dissertation \\ Presented for \\ The Graduate Studies Council \\ The University of Tennessee \\ Health Science Center
}

\begin{abstract}
In Partial Fulfillment
Of the Requirements for the Degree

Doctor of Philosophy

From The University of Tennessee
\end{abstract}

By

Satish Kumar Nandakumar

May 2014 
Copyright (C) 2014 by Satish Kumar Nandakumar. All rights reserved. 


\section{DEDICATION}

This dissertation is dedicated to my parents Nandakumar and Lakshmi Bai and my wife Hui Yu for their love and support. 


\section{ACKNOWLEDGEMENTS}

First I would like to thank my mentor Dr. Derek Persons for his guidance and support throughout my graduate training. His training has helped me become the independent and confident scientist that I am today. I would like to thank all the members of the Persons Lab especially Dr. Kyle Johnson, Dr. Tamara Pestina, Philip Hargrove and Dr. Hui Fen Zhao for providing me with technical assistance along the way.

I would like to thank my committee members Dr. Brian Sorrentino, Dr. Gerard Zambetti, Dr. Erin Schuetz and Dr. John Cox for their guidance and constructive criticism. Special thanks to Dr. Gerard Zambetti and Dr. Brian Sorrentino for their guidance and career advice during the final stages of my graduation.

I am grateful to Dr. Shannon McKinney-Freeman and Dr. Wilson Clements for including me in their inspiring lab meetings and for their guidance during my post-doc search. Attending their lab meetings made me realize how the mouse and zebrafish model systems complement each other in studying blood development. I would also like to thank Dr. Per Holmfeldt for his critique on my graduate project.

Finally I would like to thank my loving wife, Hui Yu for all her encouragement, love and support during my graduate studies. She has been my inspiration and motivation all along. I would also like to thank my mom, dad and my sister for their love and support. 


\begin{abstract}
The transcription factor GATA2 is highly expressed in hematopoietic stem cells (HSCs) and is downregulated during differentiation. Overexpression of GATA2 is frequently observed in acute myeloid leukemia. In previous studies, enforced expression of GATA2 using a MSCV GATA2 retroviral vector blocked differentiation of HSC and progenitors without inducing leukemia. We hypothesized that a lower dose of GATA2 can relieve the HSC block and eventually transform myeloid progenitors into leukemia stem cells. To test this hypothesis we generated a MSCV GATA2-ERT vector in which nuclear concentration of GATA2 can be regulated by Tamoxifen (TAM). The GATA2-ERT protein was confirmed to leak into nucleus of transduced BM cells even in the absence of TAM. This low nuclear GATA2-ERT led to enhanced self-renewal of myeloid progenitors in vitro and was able to immortalize primary bone marrow (BM) cells into IL-3 dependent myeloid cell lines. Continuous GATA2-ERT expression was also required for the proliferation of these immortalized lines. Nmyc and HoxA9 mRNA were significantly higher in GATA2-ERT immortalized lines compared to control immortalized lines. Repression of either Nmyc or Hoxa9 in GATA2-ERT immortalized cells impaired their proliferation suggesting that they are potential GATA2 targets mediating this effect.
\end{abstract}

Myeloid expansion and a block in T cell and B cell lineage differentiation was observed in transplant recipients of GATA2-ERT expressing BM cells without TAM. The myeloid expansion occurs after the Granulocyte Monocyte Progenitor (GMP) and the lymphoid block occurs after the Common Lymphoid Progenitor (CLP) in transgenic mice. Finally using the C352P mutant we showed that myeloid expansion and B lymphoid block require DNA binding activity of GATA2-ERT and were not due to the dominant negative effect of the cytosolic form of this protein.

We were the first to demonstrate that GATA2 overexpression confers increased self-renewal of myeloid progenitors which correlates with increased GATA2 mRNA levels frequently observed in human AML patients. We believe that the lower dose of GATA2 is critical to this phenotype. Interestingly no myeloid leukemias were observed in both the transplant and transgenic animals. 


\section{TABLE OF CONTENTS}

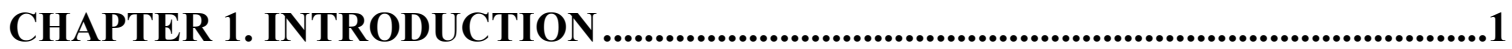

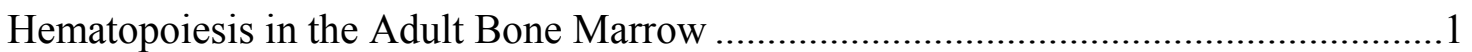

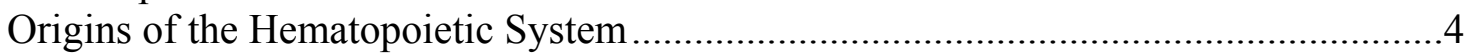

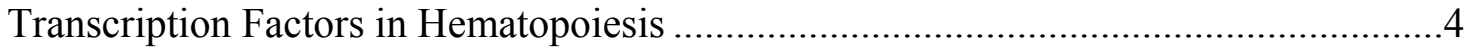

GATA Family of Transcription Factors ……………….........................................6

GATA2 Gene Structure and Cis-Regulatory Elements ...............................................

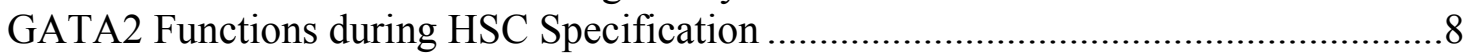

GATA2 Functions during Differentiation and Self-Renewal ......................................10

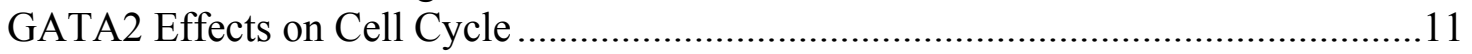

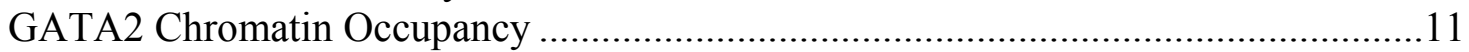

GATA2 Mutations in Acute Myeloid Leukemia .............................................................13

CHAPTER 2. MATERIALS AND METHODS.......................................................15

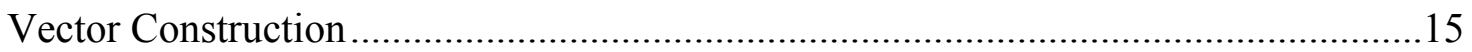

Construction of the inducible GATA2-ERT vectors ...............................................15

Construction of the GATA2 DNA binding mutants .................................................15

Construction of a lentiviral mir30 based shRNA vector............................................16

Cloning of the hairpins targeting genes of interest into the pCL20c mir30

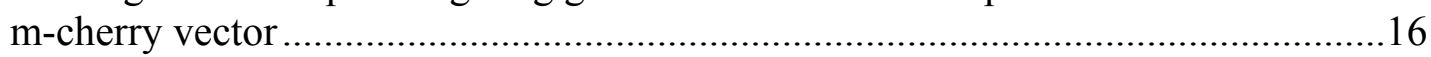

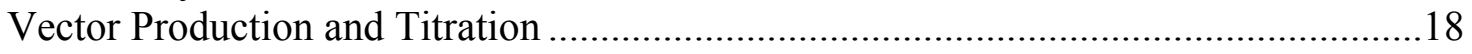

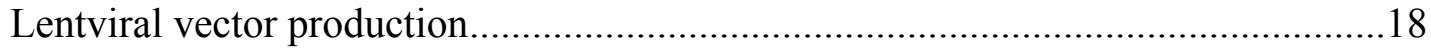

Retroviral vector production - transient prep........................................................18

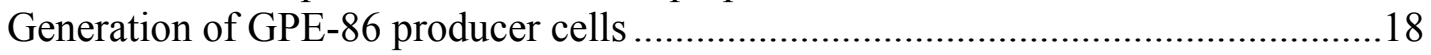

Retroviral vector production from GPE-86 producers..............................................19

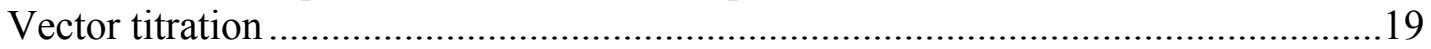

Isolation of Bone Marrow Cells and Transduction....................................................19

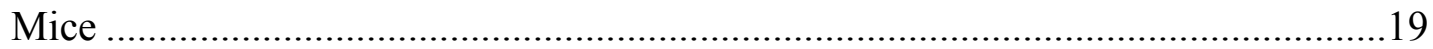

Isolation and transduction of lineage depleted mouse BM cells.................................19

Isolation and transduction of 5-FU treated bone marrow cells ...................................20

Transduction of GATA2-ERT and GFP cell lines with the lentiviral shRNA

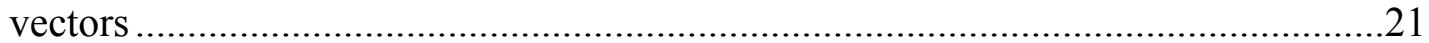

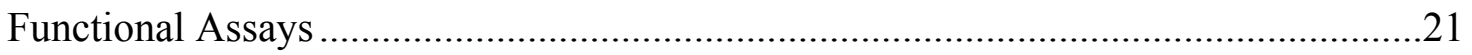

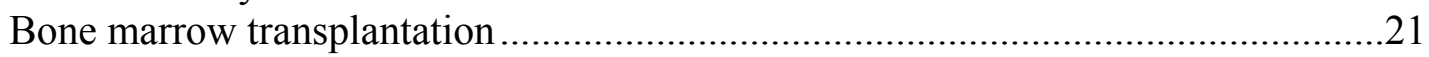

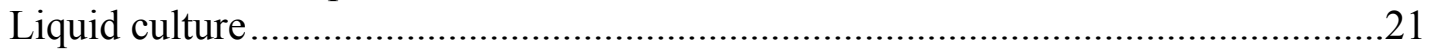

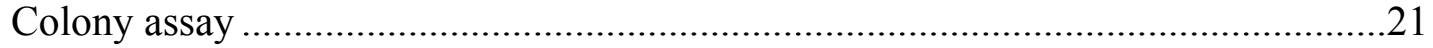

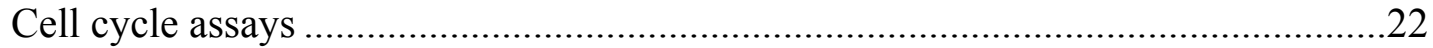

Flow cytometry and cell sorting ………………………...................................22

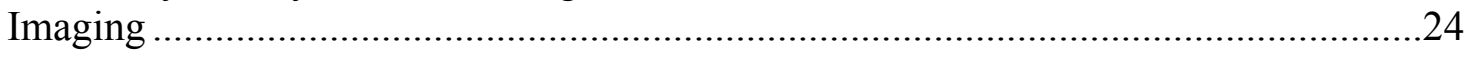

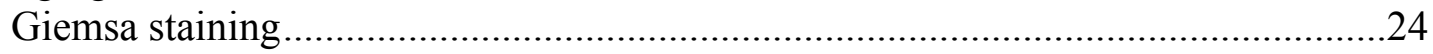

Immunostaining and confocal microscopy ……………....................................24

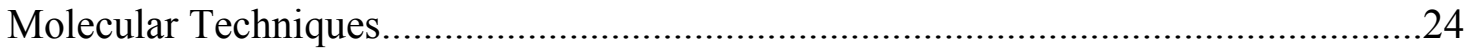

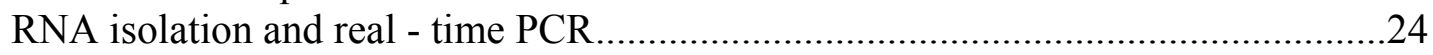

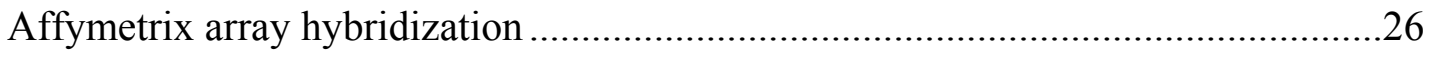


Total protein extraction using Laemelli buffer ...........................................................26

Cell compartment extraction using the Qproteome cell compartment kit .................27

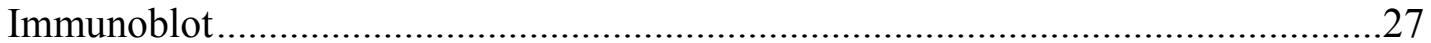

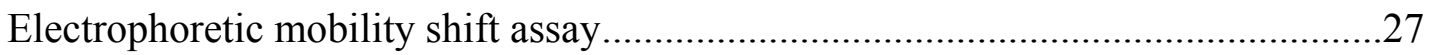

T4 kinase labeling reaction and annealing reactions .............................................28

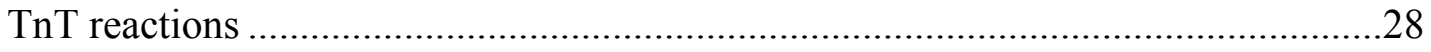

Chromatin immunoprecipitation ....................................................................28

CHAPTER 3. RESULTS..............................................................................................

Downregulation of GATA2 Expression during Hematopoiesis in the Adult BM..........31

GATA2-ERT Expressing BM Cells Exhibit TAM Independent GATA2 Function in CFU-C Assays ……………………................................................................

GATA2-ERT Vector Immortalizes Primary BM Cells into Myeloid Cell Lines............35

Low Nuclear Concentration Achieved by the GATA2-ERT Vector in the Absence

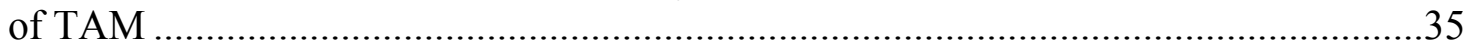

Distinct Progenitor and Mature Cell Populations Are Present in GATA2-ERT Cell

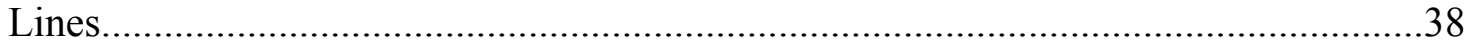

Myeloid Immortalization Is GATA2-ERT Dependent ................................................38

Screening of Genes Involved in GATA2-ERT Mediated Immortalization ....................43

HoxA9 and Nmyc Knockdown Partially Recapitulates GATA2-ERT Loss in

Immortalized Cell Lines ...............................................................................................

Absence of GATA2-ERT Chromatin Occupancy in the Nmyc and HoxA9 Locus .......51

Lymphoid Block and Myeloid Expansion in Recipients of GATA2-ERT

Transduced Cells

GATA2-ERT Blocks the Lymphoid Development after Common Lymphoid

Progenitors (CLPs) and also Expands the Granulocyte Monocyte Progenitors

(GMPs).

DNA Binding Activity of GATA2-ERT Is Required for Myeloid Expansion and

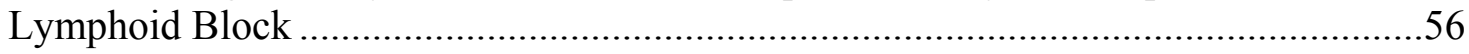

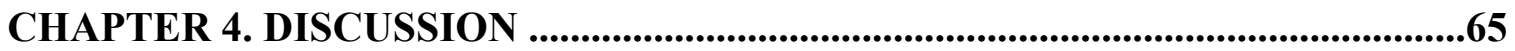

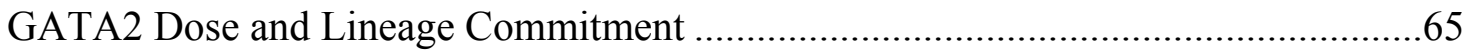

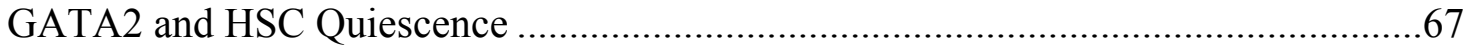

GATA2 Mediated Myeloid Expansion.....................................................................68

GATA2 Mediated Lymphoid Block …………………..................................................69

GATA2 Overexpression in Acute Myeloid Leukemia (AML) ....................................70

GATA2 Mutations in Familial AML/MDS Syndromes ..............................................71

Technical Considerations in Using the GATA2-ERT System .....................................72

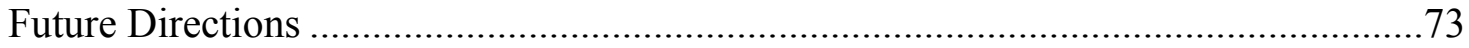

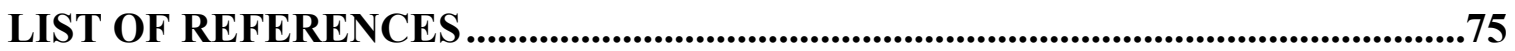

VITA....................................................................................................................................89 


\section{LIST OF TABLES}

Table 1-1. Cell surface phenotypes of different hematopoietic populations...................3

Table 1-2. GATA2 mutations in myeloid neoplasms.............................................14

Table 2-1. Primers used for mir30 based short hairpin cloning. ...............................17

Table 2-2. Antibodies used for flow cytometry and cell sorting..............................23

Table 2-3. Antibodies used for immunostaining (IHC) and western blot (WB)..........25

Table 2-4. TaqMan primer probe sets used for RT-PCR........................................26

Table 2-5. Primers used in quantitative chromatin immunoprecipitation assay............30

Table 3-1. Genes differentially expressed in GATA2-ERT vs. GFP cell line (cell proliferation).

Table 3-2. Genes differentially expressed in GATA2-ERT vs. GFP cell line (hematopoiesis)

Table 3-3. Genes differentially expressed in GATA2-ERT vs. GFP cell line (apoptosis).

Table 3-4. Changes in gene expression in GATA2-ERT expressing LSKs..................58 


\section{LIST OF FIGURES}

Figure 1-1. Proposed models for the hematopoietic hierarchy...................................2

Figure 1-2. Timeline of hematopoietic events in the mouse conceptus. .........................5

Figure 1-3. GATA2 gene structure and cis-regulatory elements................................

Figure 3-1. Endogenous GATA2 expression in various stem and progenitor compartments of the adult BM.

Figure 3-2. Successful transduction of murine BM cells with the inducible GATA2-ERT retroviral vector.

Figure 3-3. GATA2-ERT expressing cells exhibit TAM independent GATA2 function on myeloid CFU-C assays.

Figure 3-4. GATA2-ERT immortalizes primary BM cells into myeloid cell lines. .36

Figure 3-5. Effects of the absence and presence of Tamoxifen on GATA2-ERT cell lines.

Figure 3-6. Subcellular localization of GATA2-ERT before and after treatment with TAM in GATA2-ERT immortalized cell lines.

Figure 3-7. Subcellular localization of GATA2-ERT in GPE-86 producer cells.

Figure 3-8. Distinct progenitor and mature cell populations are present in GATA2ERT cell lines.

Figure 3-9. GFP targeting vector effectively knocks down GATA2-ERT protein levels.

Figure 3-10. Knockdown of GATA2-ERT inhibits proliferation of the immortalized cell lines.

Figure 3-11. Knockdown of GATA2-ERT inhibits proliferation of the immortalized cell lines due to G0/G1 cell cycle arrest.

Figure 3-12. Knockdown of GATA2-ERT inhibits proliferation of the immortalized cell lines due to differentiation.

Figure 3-13. HoxA9 and Nmyc knockdown partially recapitulates GATA2-ERT loss in immortalized cell lines.

Figure 3-14. Elimination of the mature Gr1+ population in the GATA2-ERT cell lines prevents protein degradation and improves yield. 
Figure 3-15. Absence of GATA2-ERT chromatin occupancy in the Nmyc and HoxA9 loci.

Figure 3-16. Lymphoid block and myeloid expansion in recipients of GATA2-ERT

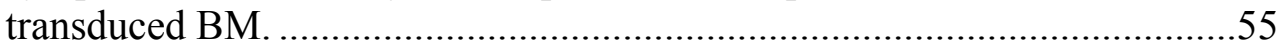

Figure 3-17. GATA2-ERT blocks lymphoid development after CLPs and also expands GMPs.

Figure 3-18. Schematic of the mutant GATA2-ERT retroviral vectors.

Figure 3-19. Expression and subcellular localization of GATA2-ERT mutants in primary BM cells.

Figure 3-20. C352P and C370P are DNA binding defective mutants of GATA2.

Figure 3-21. DNA binding activity of GATA2-ERT is required for myeloid expansion in vitro.

Figure 3-22. DNA binding activity of GATA2-ERT is required for myeloid expansion and lymphoid block in vivo. .64

Figure 4-1. Models of GATA2 dose dependent lineage commitment. .66 


\section{LIST OF ABBREVIATIONS}

AeBSF

AGM

AMKL

AML

AutoMACS

BAC

$\mathrm{BM}$

BrDU

cDNA

CFU-C

CFU-GM

CFU-S

ChIP

ChIP-PCR

ChIP-Seq

CLP

CML

CMP

DAPI

DCML syndrome

DMEM

DNA

DTT

E 7.5

E10.5

EDTA

EMSA

ER

ERT

ES cells

EtOH

FACS

FBS

FDR

GAPDH

GATA2

GFP

GMP

GP1-1

GPE-86

GSEA
4-(2-Aminoethyl) benzenesulfonyl fluoride

Aorto-gonad-mesonephros region

Acute Megakaryocytic Leukemia

Acute myeloid leukemia

Automated magnetic-activated cell sorting

Bacterial artificial chromosome

Bone marrow

5-bromo-2'-deoxyuridine

complementary DNA

Colony forming unit in culture

Colony forming unit- Granolocyte/Macrophage

Colony forming unit in spleen

Chromatin immunoprecipitation

Chromatin immunoprecipitation followed by PCR

Chromatin immunoprecipitation followed by massively parallel

DNA sequencing

Common lymphoid progenitor

Chronic Myeloid Leukemia

Common myeloid progenitor

4',6-diamidino-2-phenylindole

Dendritic cell, monocyte, B- and NK-lymphoid deficiency

Dulbecco's modified Eagle medium

Deoxyribonucleic acid

Dithiothreitol

Embryonic day 7.5

Embryonic day 10.5

Ethylenediaminetetraacetic acid

Electrophoretic mobility shift assay

Estrogen receptor (Ligand binding domain)

Estrogen receptor (Ligand binding domain -Tamoxifen specific)

Embryonic stem cells

Ethanol

Fluorescence activated cell sorting

Fetal Bovine Serum

False discovery rate

Glyceraldehyde 3-phosphate dehydrogenase

GATA binding protein 2

Green Fluorescence protein

Granulocyte monocyte progenitor

Lentiviral helper plasmid containing HIV-1 gag and pol regions

3T3 based packaging cell line containing gag pol and envelope regions of Moloney murine leukemia virus

Gene set enrichment analysis 


\begin{tabular}{|c|c|}
\hline HPV & Human papillomavirus \\
\hline HSC & Hematopoietic stem cell \\
\hline $\operatorname{IgG}$ & Immunoglobulin \\
\hline $\mathrm{IHC}$ & Immunostaining \\
\hline IL-3 & Interleukin 3 \\
\hline IL-6 & Interleukin 6 \\
\hline ires & Internal ribosome entry sequence \\
\hline $\mathrm{Kd}$ & kilodaltons \\
\hline KTLS cells & c-kit+, Thy1.1low, Lin-, Sca1+ cells \\
\hline Lin & Lineage markers (Ter119, Gr1, Mac1, CD4, CD8, B220) \\
\hline LMPP & Lymphoid primed multipotent progenitor \\
\hline LPE & Local pooled error \\
\hline LSK cells & Lin-, Sca1+c-kit+ cells \\
\hline LT-HSCs & Long term hematopoietic stem cells \\
\hline LTR & Long terminal repeats \\
\hline MDS & Myelodysplastic syndrome \\
\hline MEP & Megakaryocyte erythroid progenitor \\
\hline MFI & Mean fluorescence intensity \\
\hline $\mathrm{MgCl} 2$ & Magnesium chloride \\
\hline $\operatorname{mir} 30$ & MicroRNA 30 \\
\hline MOI & Multiplicity of infection \\
\hline MonoMAC & Monocytopenia and mycobacterial infection \\
\hline MPPs & Multipotent progenitors \\
\hline NK cell & Natural killer cell \\
\hline NS & Non silencing \\
\hline NSG & NOD SCID IL2 receptor gamma chain knockout mice \\
\hline PBS & Phosphate buffered saline \\
\hline PCR & Polymerase chain reaction \\
\hline PVDF & Polyvinylidene difluoride \\
\hline RNA & Ribonucleic acid \\
\hline RT & Room temperature \\
\hline RT-PCR & Real - Time PCR \\
\hline RTR2 & Lentiviral helper plasmid containing HIV-1 rev and tat regions \\
\hline $\mathrm{SCF}$ & Stem cell factor \\
\hline SD & Standard deviation \\
\hline SDS & Sodium dodecyl sulphate \\
\hline shRNA & Short hairpin RNA \\
\hline SLAM & Signaling lymphocyte activation molecule \\
\hline ST-HSCs & Short term hematopoietic stem cells \\
\hline TAM & Tamoxifen \\
\hline TBS & Tris buffered saline \\
\hline TBS-T & Tris buffered saline with $0.1 \%$ - Tween 20 \\
\hline TF & Transcription factor \\
\hline TnT & In vitro transcription and translation \\
\hline UG2 & Urogenital enhancer 2 \\
\hline UG4 & Urogenital enhancer 4 \\
\hline
\end{tabular}


UTR

VSVG

WB

YAC

$\mathrm{ZnCl} 2$

293T

$3 \mathrm{~T} 3$
Untranslated region

Vesicular stomatitis virus $\mathrm{G}$

Western blot

Yeast artificial chromosome

Zinc chloride

Human Embryonic Kidney 293 cells

Mouse embryonic fibroblast cell line 


\section{CHAPTER 1. INTRODUCTION}

\section{Hematopoiesis in the Adult Bone Marrow}

Bone marrow (BM) is the primary site of blood production (hematopoiesis) in both adult mice and humans. The understanding that the BM is the site of hematopoiesis came from studies on radiation injury. In 1945 Hiroshima and Nagasaki atomic bomb victims were found to have died of hematopoietic failure. ${ }^{1}$ Later it was shown that a similar radiation syndrome in mice could be prevented by BM transplantation. ${ }^{2-4}$ In 1961 , Till and McCulloch showed that BM cells transplanted into lethally irradiated mice formed spleen colonies (CFU-S) which contained a clonal population of myeloerythroid cells (granulocytes, macrophages, red cells and megakaryocytes) and cells of lymphoid potential. ${ }^{5-7}$ Some cells from these CFU-Ss can form additional CFU-S upon secondary transplantation. Till and McCulloch were the first to propose the existence of a hematopoietic stem cell (HSC) which can self-renew and differentiate into all blood cell types. This led to the search for the Hematopoietic Stem cell in the BM. Between 1988 and 1992, the Weissman group successfully isolated the murine HSC by enrichment of BM cells expressing stem cell markers (Sca-1, c-kit, Thy1.1) and depletion of cells expressing the mature "lineage markers" (Gr-1, Mac-1, B220, CD4, CD8, Ter-119 and NK1.1). ${ }^{8,9}$ Subsequently it was also shown that all the HSCs of the mouse BM were located in this c-kit+, Thy $1.1^{\text {low }}$, Lin-, Sca1+ (KTLS) compartment of the BM. ${ }^{10}$

Two models of lineage commitment from the HSC have been proposed so far (Figure 1-1). The KTLS cells are a heterogeneous population of multipotent cells with different self-renewal capacities. ${ }^{11}$ Within the KTLS cells the Long Term HSCs (LTHSCs) are the true HSCs which have the self-renewal capacity for the life-time of the organism. The LT-HSCs gives rise to the Short Term HSCs (ST-HSCs) with the selfrenewal capacity of less than 8 weeks and their progeny the Multipotent Progenitors (MPPs) with no self-renewal capacity. ${ }^{12}$ In the first model proposed by the Weissman group, the ST-HSCs or MPPs gives rise to either a Common Lymphoid Progenitor (CLP) or a Common Myeloid Progenitor (CMP) which in turn gives rise to either a Granulocyte-Macrophage Progenitor (GMP) or a Megakarocyte-Erythroid Progenitor (MEP). ${ }^{13}$ In the alternate model proposed by the Jacobsen group, the MEP is derived directly from the ST-HSCs/MPPs and a Lymphoid-Primed Multipotential Progenitor (LMPP) gives rise to the CLP and the GMP. ${ }^{14}$ The CLP, GMP, and MEP in turn differentiate into respective differentiated cells (CLP: T-cells, B-cells, NK cells; GMP: neutrophils, basophils, eosinophil's, macrophages, mast cells; MEP: Red blood cells, platelets) through intermediate progenitor stages. The T-cell progenitors are unique that they also retain the ability to differentiate into myeloid cells at early stages. ${ }^{15,16}$ All the various stem cells, progenitor cells and mature cells described above can now be isolated based on their surface marker expression (Table 1-1). Recently Orkin's group did a computational mapping based on multiplex single cell quantitative PCR analysis (280 cell surface markers) and generated a hierarchy similar to the Jacobsen model. ${ }^{17}$ Furthermore, they also demonstrated that a subset of MPPs generates preferentially megakaryocytes and RBCs showing that the Jacobsen model is more relevant. ${ }^{17}$ 


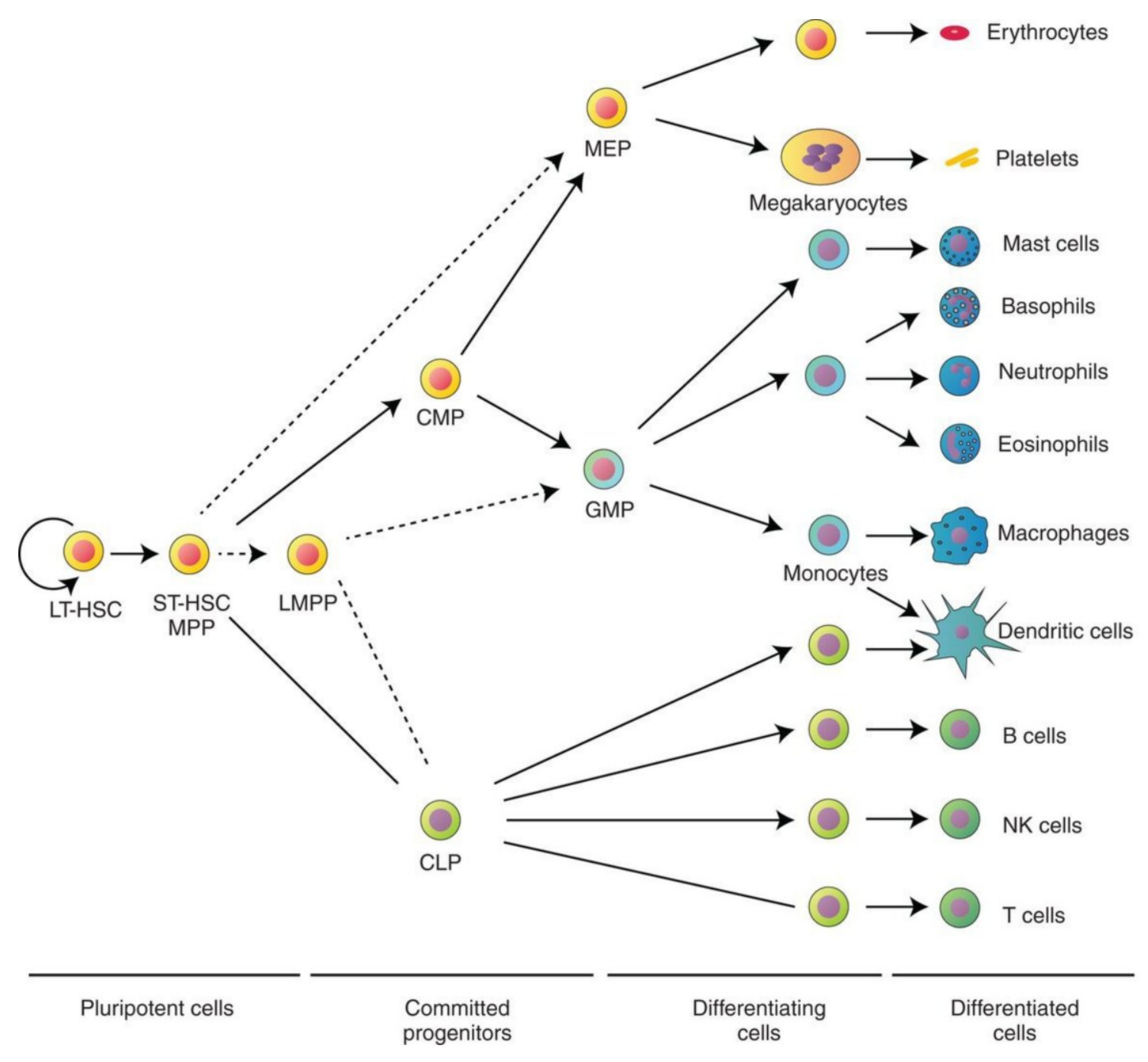

Figure 1-1. Proposed models for the hematopoietic hierarchy.

In the model proposed by the Weissman group (solid arrows), multipotential progenitors (MPPs or short-term HSCs [ST-HSCs]) give rise to either a common lymphocyte progenitor (CLP) or a common myeloid progenitor (CMP), which, in turn, gives rise to either a granulocyte-macrophage progenitor (GMP, equivalent to CFU-GM) or a megakaryocyte-erythroid progenitor (MEP). The alternate model suggested by the Jacobson group (dotted arrows) involves the generation of MEPs directly from the MPPs/ST-HSCs, whereas a lymphoid-primed multipotential progenitor (LMPP) has the potential to generate both CLPs and GMPs. LT-HSC, Long-term hematopoietic stem cell; NK cell, natural killer cell.

Reprinted with permission: Dzierzak E, Philipsen S. Erythropoiesis: development and differentiation. Cold Spring Harb Perspect Med. 2013;3(4). ${ }^{18}$ 
Table 1-1. Cell surface phenotypes of different hematopoietic populations.

\begin{tabular}{ll}
\hline Population Name & Surface Markers \\
\hline LT-HSC & Lin- c-Kit+ Sca1+ Flt3- CD34- \\
LT-HSC (SLAM) & Lin- c-Kit+ Sca1+ CD150+ CD48- \\
LT-HSC (SP) & Lin- c-Kit+ Sca1+ Hoechst side population \\
ST-HSC / MPP & Lin- c-Kit+ Sca1+ Flt3- CD34+ \\
LMPP & Lin- c-Kit+ Sca1+ Flt3+ CD34+ \\
CLP & Lin- c-Kit ${ }^{\text {low }}$ Sca1 ${ }^{\text {low }}$ Flt3 ${ }^{\text {high }}$ IL7R $7+$ \\
CMP & Lin- c-Kit+ Sca1- CD34+ Fc $\gamma R^{\text {low }}$ \\
GMP & Lin- c-Kit+ Sca1- CD34+ Fc $\gamma R+$ \\
MEP & Lin- c-Kit+ Sca1- CD34- Fc $\gamma R-$ \\
B-cells & B220 \\
T-cells & CD3, CD4 and CD8 \\
NK cells & NK1.1 \\
RBC & Ter119 \\
Platelets & CD41 \\
Granulocytes & Gr-1 \\
Macrophages & Mac-1 \\
\hline
\end{tabular}

HSC: Hematopoietic Stem Cell, LT-HSC: Long Term HSC, ST-HSC: Short Term HSC, MPP: Multipotent Progenitors, LMPP: Lymphoid primed multipotent progenitor, CLP: Common Lymphoid progenitor, CMP: Common myeloid progenitor, GMP: Granulocytemacrophage progenitor, MEP: Megakaryocyte-Erythroid progenitor, Lin: Lineage markers. 


\section{Origins of the Hematopoietic System}

Even though the BM is the primary site of hematopoiesis in the adult, the HSCs are not normally generated de-novo in the BM but are colonized during embryonic development from other sites. During embryogenesis, hematopoiesis occurs sequentially at different sites and hematopoietic cells later colonize secondary territories to finally end up in the BM (Figure 1-2). ${ }^{19}$ The first wave of hematopoiesis is detectable at embryonic day 7.5 (E 7.5) in the yolk sac blood islands. This is called the primitive hematopoiesis and it generates red blood cells that express embryonic globins. The primitive hematopoiesis is transient and is replaced by definitive hematopoiesis which is characterized by expression of adult type globins. The first site of definitive hematopoiesis is located in the Aorto-Gonado-Mesonephros (AGM) region at E10.5. In this region, "hemogenic endothelial cells" on the ventral side of the dorsal aorta bud off hematopoietic stem cells. The hematopoietic cells in the AGM region are capable of reconstituting hematopoiesis in lethally irradiated mice. ${ }^{20}$ At a similar time hematopoietic clusters are also found in the umbilical arteries, allantois and the placenta. ${ }^{21-23}$ The relative contribution of these sites to the final pool of adult HSCs is still unknown. The definitive hematopoietic cells then migrate into the fetal liver, thymus, spleen and finally the BM (Figure 1-2). The properties of HSCs in these niches are different, for example the fetal liver HSCs are cycling compared to the BM HSCs which are mostly quiescent. ${ }^{19}$

\section{Transcription Factors in Hematopoiesis}

The importance of Transcription Factors (TFs) in hematopoiesis can be appreciated by the fact that their dysregulated expression and mutations are frequently found in leukemia. ${ }^{24-26}$ Hematopoietic transcription factors can be arbitrarily classified into "HSC specific" TFs and "lineage specific" TFs. The HSC specific TFs like Scl/Tal1 and Runx-1 are required for generation of the HSC. Scl/Tall knockout mice is embryonic lethal with no blood formation in the yolk sac and embryo indicating its importance in both primitive and definitive hematopoiesis. ${ }^{27-29}$ Runx-1 knockout mice had normal primitive hematopoiesis but fail to form hematopoietic clusters in the AGM region, the first site of definitive hematopoiesis. ${ }^{30-32}$ On the other hand, Lineage specific TFs like CEBP $\alpha$ and GATA1are required for the generation of specific lineages. Mature neutrophils and eosinophils are absent in the blood and fetal liver of CEBP $\alpha^{-/-}$mice while other lineages are not affected. ${ }^{33}$ Similarly GATA1 ${ }^{-/-}$ES cells do not contribute to mature red blood cells in chimeras but do generate other non-erythroid lineages. ${ }^{34}$

However this classification is not strict and frequently HSC specific TFs also have other functions in specific lineages and the lineage specific TFs have also have functions in the HSCs. Conditional inactivation of SCL/Tall in the adult bone marrow did not affect HSC self-renewal or maintenance but specifically blocked the differentiation of erythroid and megakaryocyte lineages. ${ }^{35}$ Similarly conditional inactivation of Runx1 in the adult bone marrow specifically blocked differentiation of megakaryocytes and lymphocytes without affecting HSCs. ${ }^{36}$ Conditional inactivation of the lineage specific $\mathrm{TF}, \mathrm{CEBP} \alpha$ in the fetal liver also expanded the HSCs as shown by increased competitive 


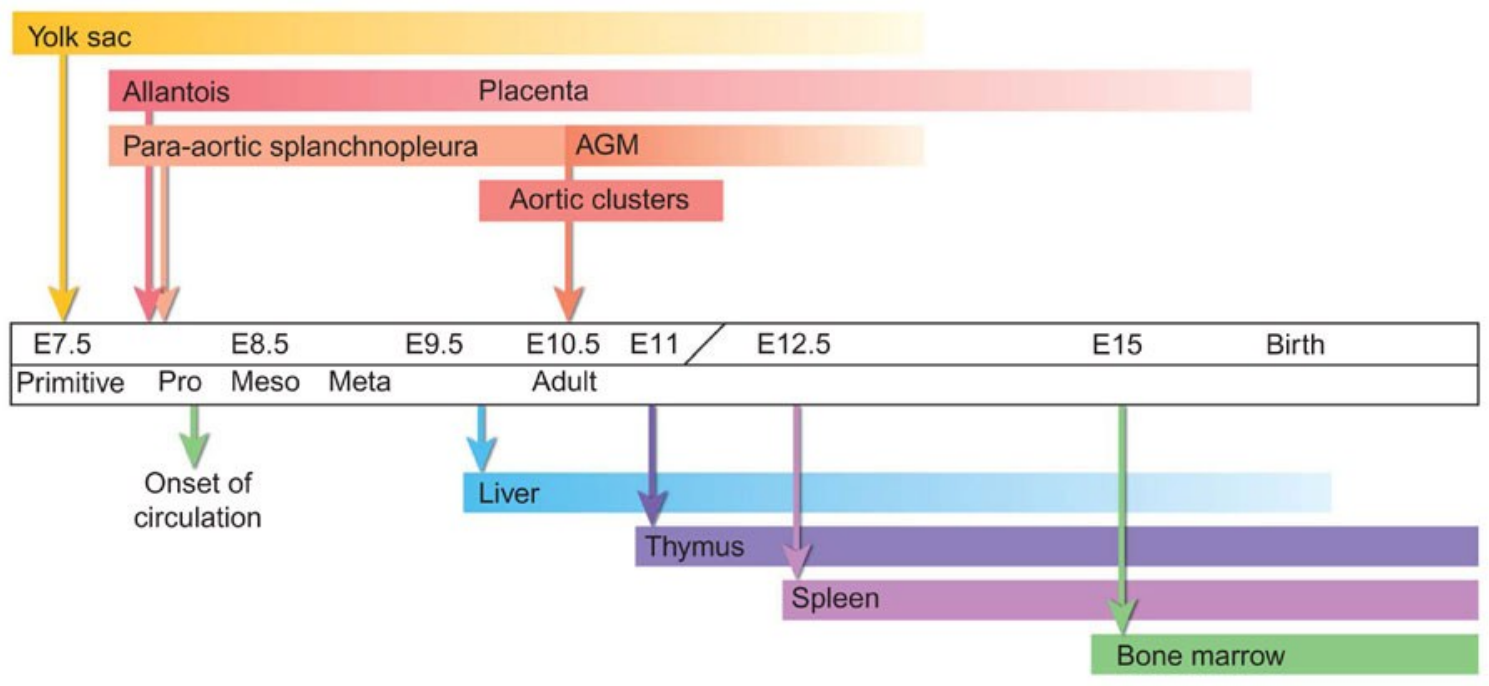

Figure 1-2. Timeline of hematopoietic events in the mouse conceptus.

Arrows above indicate the onset of specific hematopoietic cell generation and/or appearance; arrows below indicate the earliest time of colonization of the secondary hematopoietic territories. AGM, Aorto Gonado Mesonephros region.

Reprinted with permission: Dzierzak E, Speck NA. Of lineage and legacy: the development of mammalian hematopoietic stem cells. Nat Immunol. 2008;9(2):129$136 .{ }^{37}$ 
repopulation. ${ }^{38}$ This shows that lineage specific TFs may also have HSC functions. Redeployment of the same TF during different stages of development and differentiation is commonly found in hematopoiesis.

Lineage specific TFs promote their own lineage differentiation and at the same time antagonize TFs that favor other lineage choices. GATA1 and PU.1 are essential for erythroid and myeloid differentiation respectively. N-terminus region of PU.1 was shown to physically interact with the C-terminus zinc finger region of GATA1 and prevent its function by affecting the DNA binding ability. ${ }^{39,40}$ The C-terminus zinc finger of GATA1 can also block PU.1 mediated transactivation of myeloid specific genes by interacting with its ETS domain. ${ }^{41}$. Forced expression of GATA1 in myeloid cells reprograms them to undergo erythroid differentiation. ${ }^{42}$ Forced expression of PU.1 represses erythropoiesis and promotes myeloid differentiation in erythroid cell lines. ${ }^{43}$ Morpholino based knockdown of PU.1 and GATA1 in zebrafish shifts hematopoietic progenitors into erythroid and myeloid fates respectively. ${ }^{44,45}$ TFs can also antagonize each other at the level of transcription. For example during erythroid differentiation GATA1 and GATA2 antagonize each other at the transcriptional level. This phenomenon is called the "GATA switch "and will be discussed in detail later.

TFs can also mediate lineage choices in a dose dependent manner. This has been demonstrated for PU.1 and GATA1. When PU. $1^{-/-}$fetal liver cells were transduced with a PU.1iresGFP vector and cultured on OP9 cells they differentiate into macrophages and B cells. Expression of high GFP in the macrophages and low GFP in the B cells showed that PU.1 can differentiate fetal liver progenitors into different lineages based on dosage. ${ }^{46}$ Expression of GATA1 in myb-ets transformed avian myeloblasts showed a clear co-relation of GATA1 dosage and lineage choice. ${ }^{47}$ Eosinophils formed at low doses of GATA1 and erythroid, megakaryocyte development is seen at high doses.

TFs can group together with other TFs and non-DNA binding proteins to form multiprotein complexes. In erythroid cells a complex containing Scl/Tal1, E47, LMO2, Ldb1 and GATA1 has been reported. ${ }^{48}$ This complex binds to DNA sequences with composite elements containing both GATA motif (WGATAR) and E-box motif (CAANNTG). ${ }^{48}$ GATA1 also forms an alternate protein complex with its co-factor Friend of GATA (FOG). Using GATA1 mutants which cannot bind to FOG1 but have normal DNA binding activity, it has been shown that this GATA-FOG complex is essential for erythroid and megakaryocyte development. ${ }^{49}$ GATA1-FOG1 complex is further associated with the NuRD chromatin remodeling complex in both erythroid and megakaryocyte lineages through N-terminus NuRD interaction domain of FOG $1 .{ }^{50}$ This association can mediate transcriptional repression and also facilitate accessibility of the complex to its target regions.

\section{GATA Family of Transcription Factors}

The GATA binding proteins are a group of structurally related zinc finger transcription factors which bind to the consensus DNA sequence WGATAR (W=A/T, 
$\mathrm{R}=\mathrm{A} / \mathrm{G}$ ). In mammals the GATA family contains 6 members (GATA1- GATA6). GATA1, GATA2 and GATA3 are primarily expressed in the hematopoietic system. GATA1 and GATA3 are lineage specific transcription factors. GATA1 is expressed in the erythroid, megakaryocyte and eosinophil lineages whereas GATA3 is expressed in the T cell lineage. ${ }^{51}$ GATA2 is broadly expressed in the HSCs, progenitors and mast cells. ${ }^{52,53}$ These hematopoietic GATA members are also expressed in other tissues in the body. GATA1 is expressed in the Sertoli cells in testes. ${ }^{54}$ GATA2 is expressed in endothelial cells, fetal liver, fetal heart, placenta, brain and pituitary glands. ${ }^{55-59}$ GATA3 is expressed in kidney, central nervous system, placenta, skin and mammary glands. ${ }^{60-62}$ The other GATA family members, GATA4, GATA5, and GATA6 are expressed in tissues of mesodermal and endodermal origins such as heart, liver, lung, gut and gonads. $^{63}$

The functions of the hematopoietic GATA factors have been studied using knockout mice. GATA1 ${ }^{-/}$embryos die between embryonic day 10.5 and 11.5 due to a block in the differentiation of embryonic erythroid cells. GATA $1^{-/-}$ES cells in chimeras did not contribute to the erythroid lineage but had normal contribution to other lineages. ${ }^{34}$ Subsequently it was shown that targeted deletion of two control elements in the GATA1 locus lead to loss of eosinophil lineage and defects in platelet development. ${ }^{64,65}$ These experiments showed that GATA1 is required for lineage specification of erythroid, megakaryocyte and eosinophil lineages. GATA2 $2^{-/-}$embryos die between embryonic day 10 and 11 due to severe anemia. GATA2 $2^{-/-}$ES cells did not contribute to any hematopoietic cells showing a defect in HSC formation ${ }^{66} \mathrm{GATA}^{-/-}$embryos die due to internal bleeding, aberrations in fetal hematopoiesis, abnormalities in spinal cord and brain. ${ }^{67}$ GATA3 $^{-/-}$ES cells in RAG2 ${ }^{-/}$chimeras did not contribute to the T-cell lineage but had normal contribution to other lineages. ${ }^{68}$

In some instances the functions of GATA factors were shown to be partially interchangeable. When GATA3 is knocked into the endogenous GATA1 locus, the mutant mice showed a partial rescue of the GATA1 null phenotype characterized by increased survival of erythroid precursors and improved hemoglobin production. ${ }^{69}$ Transgenic expression of GATA2 and GATA3 could rescue embryonic lethality of GATA1 knockdown embryos. ${ }^{70}$ However the adult mice rescued with the transgenes developed anemia. ${ }^{70}$ In T-cells GATA3 target genes can be activated and repressed by expression of GATA1, 2 and $4 .^{71}$

The GATA factors contain two highly conserved Cys4 zinc fingers. The Cterminal zinc finger is necessary and sufficient for GATA to bind to cognate sequence WGATAR ${ }^{72,73}$ The N-terminal zinc finger can also bind to DNA sequence containing $\mathrm{GATC}^{74,75}$ and also mediates binding to co-regulator FOG1. Recently Chip-Seq experiments with GATA1,GATA2 and GATA3 factors has shown that GATA factors mostly bind to single GATA site ((A/T) GATAA) or a palindromic site (catctGATAAG) ${ }^{76-78}$ Crystal structure analysis of DNA binding domain of GATA3 revealed that the two zinc fingers can wrap around a single GATA site or bridge between two sites on different DNA molecules. ${ }^{79}$ 


\section{GATA2 Gene Structure and Cis-Regulatory Elements}

The mouse GATA2 is located on chromosome 6 spanning $8.5 \mathrm{~kb}$ and can produce two mRNA transcripts. The two transcripts differ in their $1^{\text {st }}$ exon which contains part of the 5'UTR (Figure 1-3A). The distal 1S exon is utilized in the hematopoietic and neural tissues whereas the proximal $1 \mathrm{G}$ exon is utilized in all other tissues where GATA2 is expressed. ${ }^{55,80}$ The remaining 5 exons are shared by both transcripts. Both the mRNA transcripts finally result in production of the same 480 amino acid protein.

Cis-regulatory elements that control GATA2 expression in various tissues were mapped using YAC and BAC clones containing GATA2 genomic sequences. The $\mathrm{d} 16 \mathrm{YAC}$ clone containing $-198 \mathrm{~kb}$ to $+73 \mathrm{~kb}$ region completely rescued the GATA2 ${ }^{-/-}$ hematopoietic defect whereas the $\mathrm{d} 27 \mathrm{YAC}$ clone containing $-148 \mathrm{~kb}$ to $+73 \mathrm{~kb}$ region did not. ${ }^{81}$ This localized the hematopoietic regulatory elements between $-198 \mathrm{~kb}$ to $-148 \mathrm{~kb}$ region (Figure 1-3B). Even though the d16YAC rescued the hematopoietic defect these transgenic pups die at $\mathrm{P} 0$ due to urogenital patterning defects. ${ }^{81}$ Two urogenital enhancers were then mapped to $+75 \mathrm{~kb}$ (UG4) and $+113 \mathrm{~kb}(\mathrm{UG} 2)$ region using reporter gene expression from BACs. ${ }^{82}$ Two intronic enhancer elements have been identified so far (Figure 1-3A). A $190 \mathrm{bp}$ region in the intron 5 is required for GATA2 expression in the V2 interneurons in the spinal cord. ${ }^{83} \mathrm{~A} 480 \mathrm{bp}$ region in the intron 4 drives GATA2 expression in the HSCs, vascular and lymphatic endothelial cells. ${ }^{84,85}$

Additional cis-regulatory elements called "GATA switch sites" were identified using Chromatin immunoprecipitation studies in G1E cell line (Figure 1-3B). GATA1 binds to 5 dispersed regions in the GATA2 locus $(-77 \mathrm{~kb},-3.9 \mathrm{~kb},-2.8 \mathrm{~kb},-1.8 \mathrm{~kb}$ and $+9.5 \mathrm{~kb}$ relative to the $1 \mathrm{~S}$ promoter) and repress GATA2 transcription. ${ }^{86-88}$ The same regions were previously bound by GATA 2 when it is actively expressed suggesting a positive feedback loop. Targeted deletion of the $-1.8 \mathrm{~kb}$ region reactivates GATA2 transcription in late stage erythroblasts where it is normally repressed resulting in an erythroid maturation defect. ${ }^{89}$ Targeted deletion of the $-2.8 \mathrm{~kb}$ region reduced GATA2 expression in LT-HSCs and MPPs of fetal liver without affecting their numbers. ${ }^{90}$

\section{GATA2 Functions during HSC Specification}

GATA2 functions have been studied using conventional knockout, conditional knockout and overexpression approaches. Tsai et al. generated the first GATA2 conventional knockout mice by replacing the exon5 which encodes the 2 nd zinc finger with the neo cassette. ${ }^{66}$ The GATA2 knockout mice die at E10.5 due to severe anemia. Yolk sac of these mice had fewer hematopoietic cells (3 fold reduction), and made lesser CFU-C colonies. ${ }^{53,66}$ However the erythroid differentiation was normal. These data demonstrate that primitive hematopoiesis is defective upon GATA2 removal. Defects in the definitive hematopoiesis were much more profound. Since the mice die at E10.5 definitive hematopoiesis was studied in chimeras generated by injecting GATA2 ${ }^{-/-}$ES cells into wild-type blastocysts. GATA2 ${ }^{-/-}$ES cells did not contribute to any aspects of definitive hematopoiesis (fetal liver, adult BM, thymus, spleen and peripheral blood). 
A

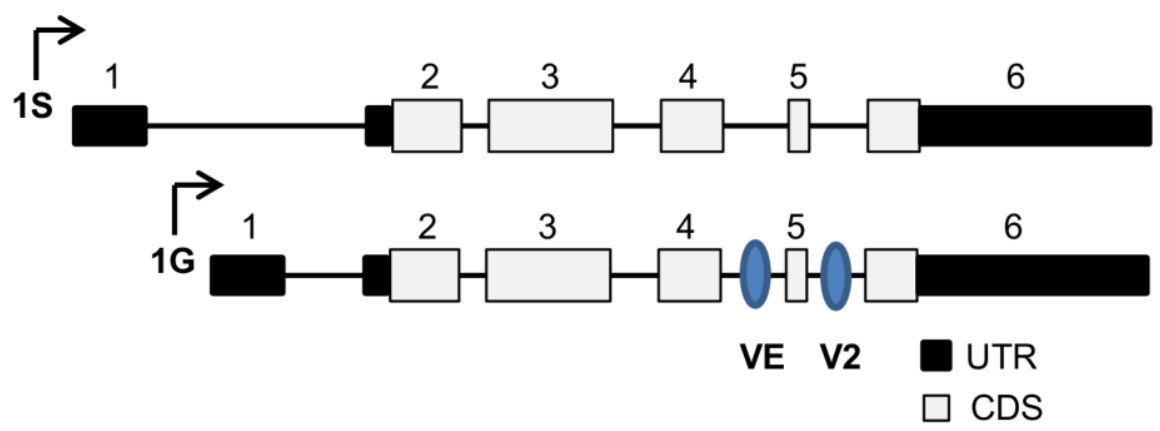

B

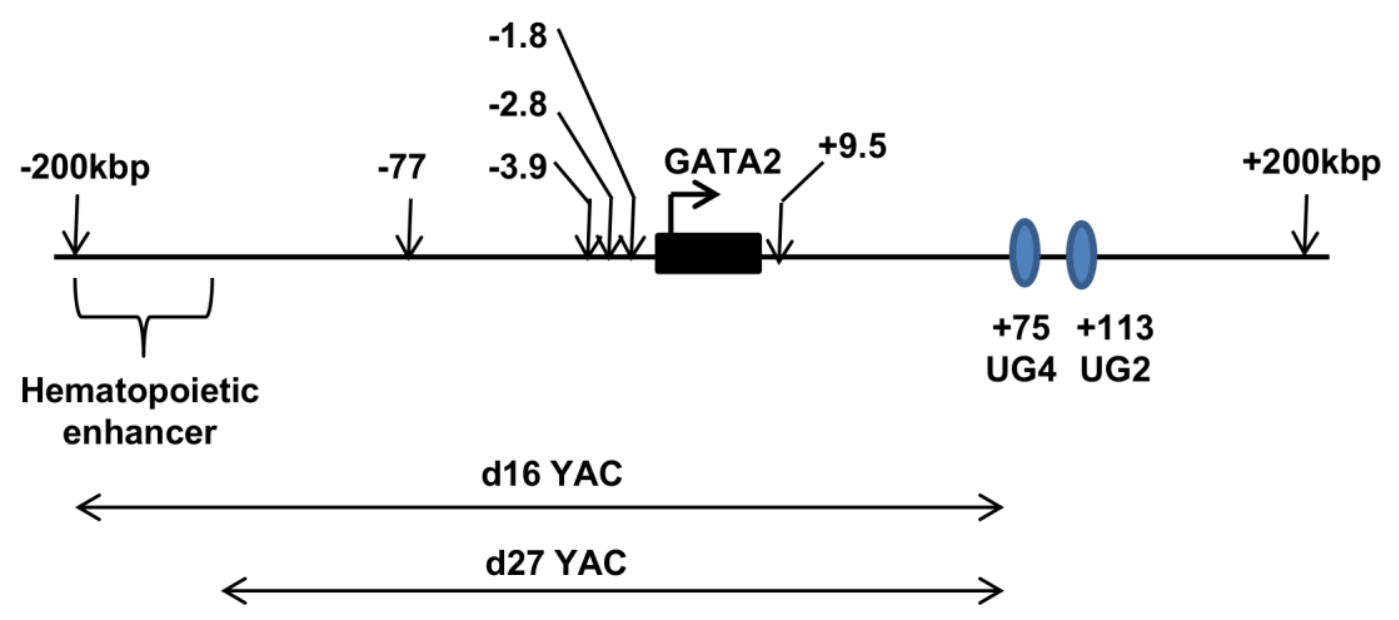

Figure 1-3. GATA2 gene structure and cis-regulatory elements.

(A) The GATA2 locus has 6 exons. The 5' and 3' untranslated region (UTR) is shown in black. Arrows indicate the transcription start sites from the $1 \mathrm{~S}$ and $1 \mathrm{G}$ exons. The location of the two intronic enhancers is shown in blue. V2: V2 interneuron enhancer, VE: vascular and lymphatic endothelial cell enhancer. (B) Diagram of the entire GATA2 locus on chromosome 6 showing location the of different enhancer elements. Position of the $\mathrm{d} 16$ and $\mathrm{d} 27$ YAC constructs is shown in reference to the GATA2 locus. The 2 urogenital enhancers UG4 and UG2 are shown in blue. Locations of GATA switch sites are indicated by downward arrows. 
These data suggested that GATA2 is required for the generation of HSC/progenitor cells.

The GATA2 ${ }^{+/-}$mice are viable with normal blood counts. ${ }^{91}$ However the number of HSCs in the AGM region and adult BM generated is lower than wild type mice. In the $\mathrm{AGM}$ region of the GATA2 ${ }^{+/}$mice there are lesser Sca1+ cells and lesser reconstitution when transplanted into sub lethally irradiated recipients. ${ }^{92}$ In the adult BM there are fewer CD34- LSK cells and a competitive repopulation defect into lethally irradiated recipients has been reported. ${ }^{91}$ Thus the generation of definitive HSCs is also affected in a dose dependent manner by GATA2.

More recently it has been shown using a conditional knockout approach that GATA2 is also essential for the generation of the fetal liver HSCs and maintenance of the adult HSCs. Cre mediated GATA2 excision at E9-E11 reduced the number of phenotypic and functional HSCs in the E14.5 fetal livers in a dose dependent manner. ${ }^{85}$ GATA2 inactivation in the adult BM also reduced the number of phenotypic HSCs. ${ }^{85}$ Effects on the functional HSCs and progenitor compartments could not be addressed because of the VE-cre which was not expressed in progenitors. When the embryonic lethality at E10.5 was surpassed in the conditional knockout, a lymphatic patterning defect was also identified. $^{85}$

\section{GATA2 Functions during Differentiation and Self-Renewal}

After HSCs are specified it appears that GATA2 downregulation is required for differentiation and self-renewal. Expression of GATA2 mRNA is highest in HSCs followed by progenitor cells and completely absent in mature cells. ${ }^{52,93,94}$ Enforced expression of GATA2 using a retroviral vector in wild type murine BM resulted in a loss of hematopoietic progenitor derived colony formation. ${ }^{95}$ GATA2 expressing Lin- Sca1+ cells (HSCs) did not expand in the BM of transplant recipients and these primitive cells did not contribute to long term multilineage reconstitution. ${ }^{95}$ Even the cells expressing GATA2 during short term reconstitution had progressively decreasing levels of GATA2 over time. ${ }^{95}$ Similar effect was also seen upon enforced expression of GATA2 in human cord blood cells transplanted into NSG mice. ${ }^{96}$

GATA2 downregulation can be mediated by the antagonistic action of other lineage specific transcription factors. This has been demonstrated in erythroid and myeloid lineages where the lineage specific transcription factors GATA1 and PU.1 downregulates GATA2. G1E cell line is derived from in vitro differentiation and immortalization of GATA $1^{-/-}$ES cells. ${ }^{97} \mathrm{G} 1 \mathrm{E}$ cells are arrested in the pro-erythroblast stage due to loss of GATA1 and they express high levels of GATA2. ${ }^{49,98}$ Upon restoration of GATA1, GATA2 is downregulated and these cells can differentiate into mature erythroid cells. ${ }^{97,98}$ GATA1 downregulates GATA2 expression by displacing GATA2 at 5 regions in the GATA2 locus itself $(-77 \mathrm{~kb},-3.9 \mathrm{~kb},-2.8 \mathrm{~kb},-1.8 \mathrm{~kb},+9.5 \mathrm{~kb})$ and disrupting its positive feedback loop. ${ }^{98}$ Similarly PU. $1^{-/-}$hematopoietic progenitors from fetal liver can be cultured in vitro and upon restoration of PU.1 can differentiate into macrophages by down regulating GATA2. ${ }^{99}$ 
GATA2 also downregulates the lineage specific transcription factors in progenitor cells. This has been observed in G1ME cells which is another cell line derived from GATA ${ }^{-/-}$ES cells. The G1ME cells are arrested in the MEP stage due to absence of GATA1. Interestingly in the G1ME cells GATA2 binds to the PU.1 locus preventing its expression. ${ }^{100}$ Accordingly, knockdown of GATA2 in G1ME cells resulted in upregulation of PU.1 and macrophage differentiation.

The only mature lineage which requires constant GATA2 expression is the mast cells. Requirement of GATA2 in this lineage was identified when GATA2 ${ }^{-/-}$yolk sac cells could not be differentiated in vitro into mast cells in the presence of SCF. ${ }^{53}$ GATA2 is also highly expressed in proliferating mast cells. ${ }^{101}$ GATA2 expression can differentiate PU.1 rescued hematopoietic progenitors into mast cells. ${ }^{99}$ GATA2 can also instruct GMP cells into mast cells when CEBPa is suppressed. ${ }^{102}$ GATA2 has also been shown to instruct eosinophil and basophil differentiation from GMPs upon sequential expression with CEBPa. ${ }^{102}$ However it is not clear whether GATA2 is required in these lineages since GATA1 is also expressed in eosinophils and basophils and GATA1 is required for the generation of the eosinophil lineage. GATA2 overexpression has also been reported to expand megakaryocytes and also implicated in Down's Syndrome AMKL associated with a GATA1short isoform. ${ }^{103-105}$

\section{GATA2 Effects on Cell Cycle}

GATA2 has also been shown to mediate cellular quiescence. GATA2 expression is higher in the quiescent HSCs than the cycling HSCs. ${ }^{96}$ It is possible that the downregulation of GATA2 brings the HSCs into cell cycle during differentiation and self-renewal. In myeloid cell lines (32D, BaF3 and FDCP Mix), enforced expression of GATA2 using a conditional GATA2-ERT system mediates quiescence. The mechanism proposed was GATA2 down-regulates c-myc, resulting in decrease in Cul1 and Skp2 (E3 ubiquitin ligase complex) and accumulation of $\mathrm{p} 21 / \mathrm{p} 27$ cell cycle inhibitors. ${ }^{106}$ It was also shown in $\mathrm{BaF} 3$ cells that this quiescent state is reversible upon removal of the ligand tamoxifen.

\section{GATA2 Chromatin Occupancy}

Two studies have reported the GATA2 chromatin occupancy in HSCs and Progenitor cells (HSPCs) .Wilson et al. did chromatin immnoprecipitation followed by deep sequencing (CHIP-SEQ) of GATA2 and 9 other transcription factors in a murine hematopoietic progenitor cell line HPC-7. ${ }^{107}$ In this study GATA2 binding regions significantly overlapped with that of other transcription factors SCL, LYL1, LMO2, and RUNX1. In addition these five transcription factors were found frequently at ERG and FLI-1 binding sites. Together these 7 transcription factors form the "HSC heptad" and their targets were enriched for genes expressed in HSCs. Li et al. did CHIP-SEQ of GATA2, SCL and Ldb1 in primary lineage negative murine bone marrow cells. ${ }^{108}$ Ldb1, an adapter protein was bound to all the genes which were occupied by GATA2 and SCL. 
This suggests that Ldb1 is an important part of the complex containing GATA2 in HSPCs.

GATA2 binding sites during differentiation of erythroid cells and megakaryocytes have been studied extensively using Chip assays on G1E and G1ME cell lines. Both the cell lines are derived from in vitro differentiation of GATA1 null ES cells. ${ }^{97,109} \mathrm{G} 1 \mathrm{E}$ cells are arrested in the pro-erythroblast stage and G1ME cells are arrested in Megakaryocyte Erythroid Progenitor stage. Upon GATA1 rescue both G1E and G1ME cells differentiate into mature erythroid and megakaryocytes respectively. Both G1E and G1ME cells initially have high expression of GATA2 and downregulate it as they differentiate due to GATA1 rescue. Chromatin immunoprecipitation studies during this process have revealed that GATA1 displaces GATA2 from binding sites in target genes. This phenomenon is called as "GATA switch" leads to altered transcription of the target genes. ${ }^{110}$ GATA switch sites were identified in genes like $\beta$-globin, GATA2, c-kit ${ }^{11}$, Lyl1 ${ }^{112}$, PU. $1^{100}$ and mir144/451 microRNA cluster. ${ }^{113}$ Few examples are show below.

In the $\beta$-globin locus of G1E cells, the initial GATA2 binding results in low level globin expression. ${ }^{114}$ Upon GATA1 rescue, GATA1 displaces GATA2 resulting in high level globin expression during erythroid differentiation. In the c-kit and GATA2 loci, the initial GATA2 binding results in active transcription of these progenitor genes. GATA2 is shown to co-occupy with SCL in these loci. During the GATA switch GATA1 replaces GATA2 and the SCL occupancy is lost resulting in transcriptional repression. In the PU.1 locus, GATA2 is co-occupied with FOG-1 resulting in transcriptional repression of a myeloid gene. During GATA switch, GATA1 replaces GATA2 interacting with FOG-1 and resulting in further repression and reinforcing erythroid differentiation. Other myeloid transcription factors like $\mathrm{MpO}, \mathrm{CEBP} \alpha$ and Hhex were also bound by GATA2 and repressed in G1ME cells. ${ }^{104}$ Knockdown of GATA2 in G1ME cells results in myeloid trans-differentiation underscoring the importance of this GATA2 mediated repression of myeloid genes in the MEP. ${ }^{100}$ GATA2 also binds to and upregulates mast cell genes in G1E cells which is shut off after GATA switch. ${ }^{115}$

ChIP-Seq analysis of K562 cell line have identified GATA2 binding to the locus of co-repressor ETO-2. ${ }^{76}$ In G1E cells GATA2 binding and activation of ETO-2 was confirmed. Interestingly upon GATA1 rescue, ETO-2 binds and represses its own locus facilitating GATA1 mediated differentiation. ChIP-Seq analysis on G1ME cells identified co-occupancy of GATA2 and ETS-1 transcription factor. ${ }^{116}$ This study also pointed out that GATA2 binding sites were enriched in the regions within genes and especially in the1st intron. ${ }^{116}$

ChIP-PCR analysis of several GATA sites in G1E cells showed that GATA2 binds only to a small proportion of WGATAR motif in complex with an E-Box motif (SCL binding site). ${ }^{117}$ High percentage of GATA2 bound sites were also bound by SCL and have a specific epigenetic signature. The GATA2 bound sites were associated with acetylated histones $\mathrm{H} 3$ and $\mathrm{H} 4$ that were dimethylated at $\mathrm{H} 3 \mathrm{~K} 4$ and $\mathrm{H} 3 \mathrm{~K} 36$, and devoid of $\mathrm{H} 3 \mathrm{~K} 9$ trimethylation. ${ }^{117}$ 


\section{GATA2 Mutations in Acute Myeloid Leukemia}

GATA2 mutations were reported in several syndromes associated with predisposition to AML and MDS (Table 1-2). Hahn et al reported two GATA2 missense mutations (T354M and T355del) associated with familial MDS and AML. ${ }^{118}$ These mutations were heterozygous in the affected individuals and there was incomplete penetrance among family members. The T354M mutant showed dominant negative effects and the T355del mutant show loss of function in in vitro experiments (reporter assays, cell lines). The T354M mutation was also reported in two other syndromes with similar clinical features called the MonoMAC syndrome and the DCML syndrome. Both the syndromes were characterized by monocytopenias, B-cell and NK cell deficiency, mycobacterial and HPV infections with predisposition to MDS and AML. There were several other overlapping mutations between these two syndromes (Table 1-2). Most of the missense mutations are located in the $2^{\text {nd }}$ zinc finger of GATA2. Others include Nterminal frame-shift mutations resulting in pre-mature stop codons and complete deletions of the $2^{\text {nd }}$ zinc finger. Using exome sequencing Ostergaard et al identified GATA2 mutations in a disease of primary lymphedema associated with MDS /AML called the Emberger syndrome. ${ }^{119}$ The mutations did not overlap with previous diseases which show that the lymphatic defects require a different set of mutations. Activating mutations of GATA2 were also found in CML blast crisis phase. Recently sequencing of the genome of pediatric non-Down's syndrome Acute Megakaryocytic leukemia (AMKL) patients identified GATA2-HoxA9 fusion. In this fusion GATA2 N-terminus lacking the zinc-fingers was fused to the homeodomain of the transcription factor HoxA9. So far none of the GATA mutations have been reintroduced into mice to test if they cause the disease. 
Table 1-2. GATA2 mutations in myeloid neoplasms.

\begin{tabular}{|c|c|c|c|}
\hline Syndromes & $\begin{array}{l}\text { Amino Acid } \\
\text { Changes }\end{array}$ & Clinical Features & References \\
\hline Familial MDS/AML & $\begin{array}{l}\text { T354M } \\
\text { T355del }\end{array}$ & $\begin{array}{l}\text { Predisposition to MDS } \\
\text { and AML }\end{array}$ & Hahn et al. ${ }^{118}$ \\
\hline $\begin{array}{l}\text { MonoMAC } \\
\text { syndrome }\end{array}$ & $\begin{array}{l}\text { R398W } \\
\text { T354M } \\
\text { G81fs } \\
\text { N371K } \\
\text { R361delRNAN } \\
\text { M1del290 } \\
\text { R396W } \\
\text { R396Q } \\
\text { D259fs } \\
\text { N317fs } \\
\text { P254L } \\
\Delta 340-381\end{array}$ & $\begin{array}{l}\text { Monocytoopenia } \\
\text { B and NK cell } \\
\text { deficiency } \\
\text { Mycobacterial and } \\
\text { Papilloma virus } \\
\text { infection } \\
\text { Predisposition to MDS } \\
\text { and AML }\end{array}$ & Hsu et al. ${ }^{120}$ \\
\hline DCML syndrome & $\begin{array}{l}\text { G200fs } \\
\text { T354M } \\
\text { R398W } \\
\Delta 340-381\end{array}$ & $\begin{array}{l}\text { Same as MonoMAC } \\
\text { syndrome }\end{array}$ & Dickinson et al. ${ }^{121}$ \\
\hline Emberger syndrome & $\begin{array}{l}\text { L105Pfs } \\
\text { R78Pfs } \\
\text { R337X } \\
\text { A341Pfs } \\
\text { C373R } \\
\text { R361L } \\
\text { A194Sfs }\end{array}$ & $\begin{array}{l}\text { Primary lymphedema } \\
\text { Cutaneous warts } \\
\text { Deafness } \\
\text { Predisposition to MDS } \\
\text { and AML }\end{array}$ & Ostergaard et al. ${ }^{119}$ \\
\hline CML blast crisis & $\begin{array}{l}\mathrm{L} 359 \mathrm{~V} \\
\Delta 341-346\end{array}$ & Blast crisis in CML & Zhang et al. ${ }^{122}$ \\
\hline Pediatric AMKL & $\begin{array}{l}\text { GATA2-HoxA9 } \\
\text { fusion }\end{array}$ & $\begin{array}{l}\text { Non Down's } \\
\text { syndrome AMKL }\end{array}$ & Gruber et al. ${ }^{123}$ \\
\hline
\end{tabular}




\section{CHAPTER 2. MATERIALS AND METHODS}

\section{Vector Construction}

\section{Construction of the inducible GATA2-ERT vectors}

MSCV-GATA2iresGFP and MSCV-iresGFP gamma-retroviral constructs have been described previously. ${ }^{95}$ To generate the MSCV-GATA2-ERTiresGFP vector, the human GATA2 cDNA was cloned into the MSCV-ERTiresGFP vector, which contains the ligand binding domain of the ERT estrogen receptor variant (G521R) which is responsive only to Tamoxifen. ${ }^{124}$ The GATA2 fragment was released from the MSCV-GATA2iresGFP vector (NcoI digest) and ligated upstream of mutant ERT (EcoRI digest) after Klenow fill-in. This cloning strategy removed the stop codon and the last aminoacid $(\mathrm{G})$ from GATA2 cDNA and generated a 7 aminoacid linker (NSDPRNE) between GATA2 and ERT. The GATA2-ERTiresGFP fragment was then moved from the gamma retroviral to the lentiviral pCL10.1 GFP vector using EcoRV and NotI sites to

generate the pCL10.1-MSCV GATA2-ERTiresGFP vector. ${ }^{125}$ The lentiviral vector was only used to generate the GATA2-ERT transgenic mice. All other experiments were performed with the gamma retroviral vector.

\section{Construction of the GATA2 DNA binding mutants}

The wild type human GATA2 cDNA was first transferred to pUC19 Shuttle vector for subsequent site-directed mutagenesis. MSCV GATA2-ERT retroviral construct was cut with EcoRI to release the hGATA2 cDNA fragment $(1.4 \mathrm{~kb})$. The pUC19 vector was linearized with EcoRI and ligated to the above hGATA2 fragment to generate the pUC19 GATA2 vector . The mutagenic primers were designed using the QuickChange primer Design application from Agilent Technologies. Wildtype reverse primers were designed to run in the opposite direction starting from the mutagenic primer. The following primers with 5 ' phosphorylation were utilized: C352P (Forward, 5'- AGCCGGCACCTGTTGTGCAAATCCTCAGACGACAAC-3'; Reverse, 5'- CTTCTGGCGGCCGACAGTCTTCGC-3'), C370P (Forward, 5'- CGGGGACCCTGTCCCCAACGCCTGTGGC-3'; Reverse, 5'- TTGGCGTTTCGGCGCCATAAGGTG-3').

The pUC19 GATA2 plasmid was linear amplified with the desired mutations using the Phusion Hot Start II DNA Polymerase (Thermo Scientific). Then the plasmid is then circularized by ligation and transformed into NEB Turbo Competent E.Coli .The pUC19- GATA2 mutants were sequence verified using the following sequencing primers: 5'- GGCTCGTTCCTGTTCAGAAG-3', 5'-CTTCTCCAAGACGCCACTGC-3'). The mutant Zinc finger domains were then switched between the retroviral vectors MSCVGATA2-ERTiresGFP and MSCV-GATA2iresGFP from the pUC19-GATA2 vectors 
using SacII and BamHI sequential digests .The mutant retroviral vectors were sequence verified again using the above primers before vector production.

The pCDNA3. 1 constructs containing the wild type and mutant GATA2 and GATA2-ERT cDNA were generated for in vitro transcription and translation for the EMSA assays. The pCDNA3.1-GATA2 constructs were generated by cloning of the GATA2 fragment from the retroviral vectors using EcoRI and EcoRV enzymes. Then the pCDNA3.1-GATA2-ERT constructs were generated by replacing the GATA2 C-terminus region of the pcDNA3.1-GATA2 vector with the GATA2 C-terminus ER from each of the retroviral GATA2-ERT vectors. SacII and EcoRV was used for the pcDNA3.1-GATA2 vector. SacII and ClaI (blunt) was used for the inserts from the MSCV-GATA2-ERT vectors.

\section{Construction of a lentiviral mir30 based shRNA vector}

LMP-RFP vector (MSCV-mir30PGK-puroiresRFP), a retroviral mir30 based shRNA vector was a kind gift from the Dr. Richard Gilberson's lab. The LMP-RFP vector is a derivative of the LMP vector from Gregory Hannon's Lab. ${ }^{126}$ The "PGK puro ires" fragment was replaced with the "PGK" fragment from the pCL-c-MNDU3 PGK GFP vector using the BlpI and NcoI restriction sites. The RFP reporter was replaced with PCR amplified m-cherry cDNA .The m-cherry cDNA was PCR amplified from the pCL20-MSCV-CreERT2-Ires-mCherry vector using the following primers: m-cherry Forward,5' - GTGAGCAAGGGCGAGGAGGATA-3'; m-cherry Reverse (ClaI) 5'-TTTTTTATCGATGCTACTTGTACAGCTCGTCCA-3'. The entire mir30 cassette with m-cherry reporter was then moved to the lentiviral backbone of pCL20cGFP vector using ClaI and EagI sites. ${ }^{127}$ Finally an XhoI site outside the viral LTR was removed to generate the pCL20c-mir30mcherry vector. This vector was used for direct single step cloning of the hairpins in the future.

\section{Cloning of the hairpins targeting genes of interest into the pCL20c mir30 m-cherry vector}

The 137 bp sequences containing the hairpins were designed using the shRNA psm2 Design from the Greg Hannon Lab website (http://katahdin.cshl.org/siRNA/RNAi.cgi?type=shRNA). 3-5 oligonucleotides were designed for each of the gene targets (Table 2-1) and single strand template PCR was performed as described previously. ${ }^{128}$ The PCR products were digested using EcoRI and XhoI sites and ligated into the unique XhoI and EcoRI sites in the pCL20c-mir30mcherry vector. VSV-G pseudotyped lentiviral vector particles were prepared using a 4-plasmid system using transient transfection of $293 \mathrm{~T}$ cells, as previously described below. 
Table 2-1. Primers used for mir30 based short hairpin cloning.

\begin{tabular}{ll}
\hline Name & Sequence \\
\hline 5'miR30PCRxhoIF & 5'-CAGAAGGCTCGAGAAGGTATATTGCTGTTGACAGTGAGCG-3' \\
3'miR30PCREcoRI & 5'-CTAAAGTAGCCCCTTGAATTCCGAGGCAGTAGGCA-3' \\
F & \\
Sequencing primer & 5'-CCCTTGAACCTCCTCGTTCGACC-3 \\
shNmyc & 5'-TGCTGTTGACAGTGAGCGACCTGGCGAGCTGATCCTCAAGTAGTGAAGCCACAGATGTACTT \\
& GAGGATCAGCTCGCCAGGCTGCCTACTGCCTCGGA-3' \\
shHoxA9 & 5'-TGCTGTTGACAGTGAGCGCGGAGTTTCTGTAACATGTATAGTGAAGCCACAGATGTATACA \\
shGFP & TGTTAAACAGAAACTCCTTGCCTACTGCCTCGGA-3' \\
& 5'-TGCTGTTGACAGTGAGCGAGCACAAGCTGGAGTACAACTATAGTGAAGCCACAGATGTATAG \\
NS & TTGTACTCCAGCTTGTGCCTGCCTACTGCCTCGGA-3' \\
& 5'-TGCTGTTGACAGTGAGCGATCTCGCTTGGGCGAGAGTAAGTAGTGAAGCCACAGATGTACTT \\
\hline
\end{tabular}

The "sense-loop-antisense" regions of the 97bp "mir30-like "DNA oligonucleotides are highlighted in "blue-red-blue" format. The 5'miR30PCRxhoIF and 3'miR30PCREcoRIF were used to perform single strand template PCR "NS" denotes non-silencing vector. 


\section{Vector Production and Titration}

\section{Lentviral vector production}

We seeded $293 \mathrm{~T}$ cells at about $15 \times 10^{6}$ cells per $10 \mathrm{~cm}$ plate in the morning. Six hours after plating the cells, lentiviral helper plasmids and the vector plasmids were transfected using calcium phosphate method. The amounts of the plasmids used per $10 \mathrm{~cm}$ plate are Vector $-10 \mu \mathrm{g}$; GP1-1 $-6 \mu \mathrm{g}$; RTR2 $-2 \mu \mathrm{g}$ and VSVG $-2 \mu \mathrm{g}$. Ten plates were transfected at a single time. In a $50 \mathrm{ml}$ conical tube, plasmids required for 10 plates were mixed with $500 \mu \mathrm{l}$ of calcium phosphate and water to a total volume of $5 \mathrm{ml}$. Then $5 \mathrm{ml}$ of $2 \times$ HSBSS solution was added drop by drop while continuously vortexing the tube. The tube was then allowed to sit for 10-15 minutes. One $\mathrm{ml}$ of the transfectant was added to each of the $10 \mathrm{~cm}$ plates and mixed by tilting. The plates were incubated overnight and media was replaced with DMEM and 10\% Fetal Bovine Serum (FBS). Twenty four hours after replacing the media the virus supernatant was collected from the plates and pooled. The supernatant was filtered through a 0.22 micron filter (Steriflip) to remove any cell debris. Aliquots of the filtered virus supernatant were then flash frozen in dry ice and stored at $-80^{\circ} \mathrm{C}$. A small aliquot was taken to titer the virus at this time on 293 T cells.

\section{Retroviral vector production - transient prep}

We seeded 293 T cells at about $8 \times 10^{6}$ cells per $10 \mathrm{~cm}$ plate in the morning. Six hours after plating the cells, retroviral helper plasmids and the vector plasmids were transfected using calcium phosphate method as described above for the lentiviral production. The amounts of the plasmids used per $10 \mathrm{~cm}$ plate are Vector- $10 \mu \mathrm{g}$, Old gag pol- $5 \mu \mathrm{g}$, VSVG- $1 \mu \mathrm{g}$. The transfected plates were incubated overnight and media was replaced with DMEM and 10\% Fetal Bovine Serum (FBS). Forty eight hours after replacing the media the virus supernatant was collected from the plates and pooled.The supernatant was filtered through a 0.22 micron filter (Steriflip) to remove any cell debris. The virus supernatant was stored in ice and used for transduction of GPE-86 producer cells in the following days.

\section{Generation of GPE-86 producer cells}

We seeded $0.5 \times 10^{6} \mathrm{GPE}-86$ cells in a $10 \mathrm{~cm}$ plate. After 6 hours $5 \mathrm{ml}$ of the media was removed and replaced with $5 \mathrm{ml}$ of viral supernatant with polybrene. Every 12 hours the media was replaced with $5 \mathrm{ml}$ of viral supernatant and $5 \mathrm{ml}$ of fresh media. The transduction was carried out for 7 days and then evaluated by flow cytometry. Usually more than $90 \%$ of producer cells were transduced at this time and they were frozen down for future retroviral production. 


\section{Retroviral vector production from GPE-86 producers}

The producers were thawed and split at least 3 times before collecting the virus. Virus supernatants were collected 48 hours after the media change. The supernatants were filtered and frozen down in $-80^{\circ} \mathrm{C}$. A small aliquot is taken to titer the virus at this time on 3T3s.

\section{Vector titration}

We seeded 293T or 3T3 cells at a concentration of $0.5 \times 10^{6}$ cells per $10 \mathrm{~cm}$ plate. Six hours after seeding increasing amounts of the lentiviral or retroviral supernatant was added to each of the plates. The plates were transferred to a $\mathrm{CO}_{2}$ incubator. After 4 days the cells were lifted using trypsin and percentages of GFP positive cells (GFP \%) were analyzed using Flow Cytometry. The virus titer represented as Transducing units $/ \mathrm{ml}$ $(\mathrm{TU} / \mathrm{ml})$ is calculated using the following formula.

$$
\text { Viral Titer }(\mathrm{TU} / \mathrm{ml})=\frac{\left(0.5 \times 10^{6}\right) \times(\mathrm{GFP} \%)}{\text { volume of viral supernatant }(\mathrm{ml})}
$$

\section{Isolation of Bone Marrow Cells and Transduction}

\section{Mice}

The GATA2-ERT transgenic mice were generated by transduction of murine blastocysts with lentiviral pCL10.1 MSCV GATA2-ERTiresGFP vector supernatants. The GATA2-ERT transgenic mice were routinely identified by measuring the GFP marking in lymphoid and myeloid cells of peripheral blood. All the other experiments were performed on 8 -12 weeks old female C57Bl/6 mice (Jackson Laboratories, Bar Harbor, ME). All experimentation with mice was performed under protocols approved by the Institutional Animal Care and Use Committee of St Jude Children's Research Hospital.

\section{Isolation and transduction of lineage depleted mouse BM cells}

Bone marrow was harvested from 8 to 12 weeks old female C57BL/6J mice (The Jackson Laboratory, Bar Harbor, ME). Mice were sacrificed in the $\mathrm{CO}_{2}$ chamber and then rinsed with $70 \%$ Ethanol. Tibiae and femurs were dissected out removing the muscles and skin tissues. The ends of these bones were trimmed using scissors. The bone marrow was then flushed with $2 \%$ FBS in PBS using a $25 \mathrm{G}$ needle for the femur and $27 \mathrm{G}$ needle for the tibia. The bone marrow collected from all the bones was then flushed one more time using the $19 \mathrm{G}$ needle and then filtered through $40 \mu$ filter. The cells were then counted using methylene blue and $1 \%$ acetic acid to exclude the RBCs. The cells were 
spun down in the centrifuge at $1200 \mathrm{rpm}$ for 10 minutes. After removing the supernatant by suction the cell pellet was resuspended in AutoMACS buffer $\left(40 \mu 1\right.$ per $10^{7}$ cells). The biotin antibody cocktail which contains biotinylated antibodies against mouse CD5, CD45R (B220), CD11b (Mac1), Gr-1 (Ly6G/C), 7-4 and Ter-119 was then added (10 $\mu 1$ per $10^{7}$ cells). The cells were mixed well and incubated at $4^{\circ} \mathrm{C}$ for 10 minutes. This was followed by addition of AutoMACS buffer ( $30 \mu 1$ per $10^{7}$ cells) and anti-biotin microBeads ( $20 \mu \mathrm{l}$ per $10^{7}$ cells). The beads were mixed well and incubated for an additional 15 minutes at $4^{\circ} \mathrm{C}$. The cells were washed by adding more AutoMACS buffer ( $2 \mathrm{ml}$ per $10^{7}$ cells) and centrifuged at $1200 \mathrm{rpm} 10$ minutes. After removing the supernatant by suction the cell pellet was resuspended in AutoMACS buffer $(3 \mathrm{ml}$ for $2 \mathrm{X} 10^{8}$ cells). The lineage depleted cells were separated using the AutoMACS ${ }^{\mathrm{TM}}$ separator using the "depletes" setting. The purity of these cells was tested by staining the cells for c-kit and anti-biotin antibodies followed by flow cytometry and consistently achieved 95$99 \%$ purity.

The lineage depleted cells were prestimulated for two days in the presence of $20 \mathrm{ng} / \mathrm{ml}$ recombinant mouse IL-3, $50 \mathrm{ng} / \mathrm{ml}$ recombinant human IL-6 and $50 \mathrm{ng} / \mathrm{ml}$ recombinant mouse SCF (PeproTech) and Dulbecco's modified Eagle medium (DMEM) supplemented with $15 \%$ fetal bovine serum (FBS), 100 units $/ \mathrm{ml}$ penicillin, $100 \mu \mathrm{g} / \mathrm{ml}$ streptomycin, $2 \mathrm{mM}$ L-glutamine (R\&D systems, Minneapolis, MN). After prestimulation the cells were transferred into plates coated with $25 \mathrm{ug} / \mathrm{ml}$ of retronectin (Takara, Madison, WI).The viral supernatant was added at multiplicity of infection (MOI) between 1.5 - 3 every 12 hours. After four rounds of transduction the cells were lifted from the retronectin coated plates by scraping. The cells were directly transplanted into lethally irradiated recipients or cultured for an additional 48 hours before performing in vitro experiments.

\section{Isolation and transduction of 5-FU treated bone marrow cells}

We advocated 5-FU intra-peritoneally to mice at a dose of $150 \mathrm{mg} / \mathrm{kg}$. Two days after 5-FU administration bone marrow was isolated, filtered and counted as described above. The bone marrow cells were plated at a concentration of $2 \times 10^{6}$ cells $/ \mathrm{ml}$ with DMEM and 15\% FBS and cytokines (IL-3 - $20 \mathrm{ng} / \mathrm{ml}$, IL-6 $50 \mathrm{ng} / \mathrm{ml}$, SCF $50 \mathrm{ng} / \mathrm{ml}$ ) for prestimulation. After 24 hours of prestimulation the cells were lifted by scraping and counted. We plated $20 \times 10^{6}$ cells in retronectin coated $15 \mathrm{~cm}$ plates with virus supernatant and fresh media containing cytokines and protamine sulphate. Every 12 hours the media was replaced with fresh viral supernatant. Four transductions were carried out by this method. Six hours after the last transduction the cells were lifted by scraping and transplanted into lethally irradiated recipient mice. A small fraction of cells were cultured for another $48 \mathrm{hrs}$ and assessed for bulk transduction by flow cytometry. 


\section{Transduction of GATA2-ERT and GFP cell lines with the lentiviral shRNA vectors}

We transduced $1 \times 10^{6}$ cells with the viral supernatant at a MOI of 15-20 in retronectin coated 6 -well plates. The cells were transduced every 24 hours for a total of three hits. One day after the last transduction the cells were lifted and re-plated into suspension plates. After 48 hours the $\mathrm{m}$-cherry $\%$ was determined and the liquid culture started.

\section{Functional Assays}

\section{Bone marrow transplantation}

Transduced BM cells were harvested and either diluted with untransduced cells or kept undiluted in PBS with 2\% FBS. Female C57Bl/6 mice between 10 to 12 weeks of age were given a total body irradiation of $1125 \mathrm{cGy}$ from a ${ }^{137} \mathrm{Cs}$ source (J.L. Shepherd, San Fernando, CA, USA). We injected $2.5 \times 10^{6}$ cells in a volume of $0.5 \mathrm{ml}$ as a single dose by tail -vein injection to each recipient. All the transplant recipients received antibacterial prophylaxis (Baytril 2.27\% solution Bayer) for three weeks post transplantation. Peripheral blood analysis on the recipients was performed at 8, 12, 16 and 20 weeks posttransplant.

\section{Liquid culture}

The liquid culture experiments were initiated 48 hours after the last transduction. Cells were lifted from the plates and counted. The percentages of the transduced cells were determined by flow cytometry. For the in vitro competition experiments the transduced cells were diluted with mock cells to start at a uniform GFP percentage of $20 \%$. For the knockdown experiments no dilution was performed. Cells were plated at a starting concentration of $3.3 \times 10^{5}$ cells $/ \mathrm{ml}$. Every 3 days the cells were counted, percentages of transduced cells were measured by flow cytometry and replated again at $3.3 \times 10^{5}$ cells $/ \mathrm{ml}$. The cells were cultured in DMEM with $15 \%$ FBS and cytokines (murine IL-3 $20 \mathrm{ng} / \mathrm{ml}$, human IL-6 $50 \mathrm{ng} / \mathrm{ml}$ and murine SCF $50 \mathrm{ng} / \mathrm{ml}$ ).

\section{Colony assay}

Fifteen $\mathrm{ml}$ conical tubes containing three $\mathrm{ml}$ aliquots of Methocult GF M3434 (Stem Cell Technologies) were thawed at room temperature or $37^{\circ} \mathrm{C}$ water bath. For plating primary colonies $0.3 \mathrm{ml}$ cell suspension containing $3.75 \times 10^{3}$ cells was added to the $3 \mathrm{ml}$ of M3434 media. The tube was vortexed and allowed to stand for 5 minutes to dissipate the bubbles. Total volume of $1.1 \mathrm{ml}$ media containing cells was dispensed into two $35 \mathrm{~mm}$ plates using a 16 gauge blunt needle and 3cc syringe. After 7 days the plates were counted in a microscope with $4 \times$ objective for total colonies and GFP positive 
colonies. For subsequent re-plating the colonies were disrupted into single cell suspension by adding $2.5 \mathrm{ml}$ of DMEM and flushing through a 19G needle. The cells were washed using $30 \mathrm{ml}$ of DMEM and spun down at $1200 \mathrm{rpm}$ for 10 minutes. The cells were resupsended in a smaller volume $(0.5-1 \mathrm{ml})$ and counted. The cells were replated at $2.5 \times 10^{4}$ cells / plate and counted for colonies after 7 days.

\section{Cell cycle assays}

GATA2-ERT cell lines cultured with $100 \mathrm{nM}$ Tamoxifen or Ethanol were stained for DNA content using propidium iodide (PI) at the time points indicated. One million cells were spun down, washed once with PBS and lysed in one $\mathrm{ml}$ hypotonic solution containing PI and detergent $(0.05 \mathrm{mg} / \mathrm{ml}$ propidium iodide, $0.1 \%$ (w/v) sodium citrate, $0.1 \%(\mathrm{v} / \mathrm{v})$ Triton X-100) by vortex. Samples were then treated with DNAse-free RNAse (Calbiochem cat \# 556746) for 30 minutes at room temperature. Samples were then filtered through $40 \mathrm{u}$ mesh and analyzed by flow cytometry. We also performed cell cycle analysis on the shRNA vector transduced GATA2-ERT cell lines by incubating with Hoechst 33342 at $10 \mu \mathrm{g} / \mathrm{ml}$ for 1 hour followed by FACS analysis. ModFit Analysis (Verify Software House) was performed on total population (PI method) or select gated population (Hoechst method).

\section{Flow cytometry and cell sorting}

Single-cell suspensions were prepared from thymus and bone marrow (femoral and tibial bones) by passage through a 70-micron cell strainer (BD). Cells were resuspended in PBS with $2 \%$ FCS. About $70 \mu 1$ of blood was obtained from mice by retro-orbital puncture and RBC lysis was performed with BD FACS Lyse Wash Assistant. Cells were stained for $30 \mathrm{~min}$ with fluorochrome-conjugated or biotinconjugated antibodies. Cells were then washed with excess medium and were resuspended in PBS with 2\% FCS containing a dead cell marker (PI or DAPI) or stained again with secondary antibodies. FACSCalibur, LSR and LSRII (BD Biosciences), were used for flow cytometry and BD Aria (BD Biosciences) was used for cell sorting. For sorting LSK cells, BM from GATA2-ERT transgenic mice was enriched for Sca1 and c-kit markers using anti-PE, anti-APC magnetic beads in AutoMACS before staining with Lineage-PECy7 antibodies. For sorting individual progenitor and stem cell populations $\mathrm{BM}$ from $\mathrm{C} 57 \mathrm{Bl} / 6$ mice were lineage depleted as described above prior to staining with other progenitor and stem cell markers. Antibodies used are shown in Table 2-2. 
Table 2-2. Antibodies used for flow cytometry and cell sorting.

\begin{tabular}{llll}
\hline Epitope & Clone & Conjugate & Company \\
\hline CD3e & 145-2C11 & APC & BD \\
CD3c & 17-A2 & PE & BD \\
B220 & RA3-6B2 & eFlour605, PEcy7 & BD \\
Mac1 & M1/70 & APC-Cy7, Alexa-700, PE-Cy7 & BD \\
Gr-1 & RB6-8C5 & APC, APC-Cy7, PE-Cy7 & BD \\
CD34 & RAM34 & PE & BD \\
FcyR & $2.4 G 2$ & Alexa-700 & BD \\
Sca1 & E13-161.7 & PE, Biotin & BD \\
c-kit & 2B8 & APC, APC-Cy7 & BD \\
CD127 & A7R34 & APC & BD \\
Streptavidin & & PE Texas Red, PE-Cy7 & BD \\
CD44 & 1M7 & PE-Cy7 & BD \\
CD25 & PC61 & APC & BD \\
CD4 & L3T4 & APC-Cy7, PE-Cy7 & BD \\
CD8a & 53-6.7 & Alexa700, PE-Cy7 & BD \\
Ter119 & TER119 & PE-Cy7 & BD \\
FceR1 & MAR-1 & Biotin & Ebioscience \\
\hline
\end{tabular}




\section{Imaging}

\section{Giemsa staining}

We pelleted $5 \times 10^{4}$ cells in a $1.5 \mathrm{ml}$ tube and resuspended them in $150 \mu \mathrm{l}$ of PBS in $2 \%$ FBS. The cytoslide (Thermo Scientific \# 59910052) was setup against the cytofunnel (Thermo Scientific \#1102548) and locked using the cytoclip (Thermo Scientific \# 59910052). The cell suspension was then loaded into the cytofunnel and spun in the Cytocentrifuge at $1000 \mathrm{rpm}$ for 5 minutes (Cytospin 3 - Shandon cytocentrifuge). The cytoslide was removed from the cytoclip and stained by dipping into a staining jar containing Wright Giemsa Solution (CS434 Fisher) for $1 \mathrm{~min}$. The cyotoslide was then washed by dipping in distilled water 3 times 1 min each. The slide was then dried overnight and the coverslip was mounted after adding a drop of Cytoseal 60 (Thermo scientific). Images were taken using a Nikon microscope in $20 \times$ objective or $40 \times$ oil objectives respectively.

\section{Immunostaining and confocal microscopy}

Coverslips (22 X 22-1 Fisherbrand) were placed in 6-well plates and coated with retronectin for 2 hours at room temperature. Retronectin coated coverslips were washed once with PBS and incubated with cells at $37^{\circ} \mathrm{C}$ for 40 minutes. After cell attachment the coverslips were washed twice with PBS and fixed with 4\% paraformaldehyde for 30 minutes. Coverslips were washed again twice with PBS and permeablized with $0.1 \%$ Triton X-100 for 15 minutes. Edges of the coverslips were then dabbed in paper and blocked with Fx-signal enhancer (Invitrogen) for 30 minutes. All antibodies were prepared in 10\% normal goat serum in PBS. Coverslips were incubated with primary antibody $(1: 50)$ overnight at $4{ }^{\circ} \mathrm{C}$. Coverslips were washed three times by dipping in beakers containing PBS. Secondary antibody (1:50) was incubated 1 hour at RT. After 3 washes coverslips were incubated with $0.2 \mu \mathrm{g} / \mathrm{ml}$ of DAPI for 10 minutes. After wash with water the coverslips were mounted into glass slides (Fisherbrand Superfrost Plus) using Prolong Gold with DAPI mounting media (Invitrogen). Slides were cured overnight at room temperature. Edges were secured by applying nail polish and visualized in Zeiss LSM 510 NLO Meta confocal microscope. Antibodies used are shown in Table 2-3.

\section{Molecular Techniques}

\section{RNA isolation and real - time PCR}

When the starting cell numbers was more than $0.5 \times 10^{6}$ cells, Ribopure Kit (Ambion AM1924) was used to extract RNA. RNAqueous-Micro Kit (Ambion AM1931) was used when the starting cell numbers was less than $0.5 \times 10^{6}$ cells. RNA extraction was performed according to the kit protocols. The RNA concentration and purity was 
Table 2-3. Antibodies used for immunostaining (IHC) and western blot (WB).

\begin{tabular}{llll}
\hline Name & Dilution & Company & $\begin{array}{l}\text { Catalog } \\
\text { Number }\end{array}$ \\
\hline aGATA2 & $1: 1000(\mathrm{WB})$, & Santa Cruz & sc-9008 \\
& $1: 50(\mathrm{IHC})$ & & \\
$\alpha$ ER alpha & $1: 1000(\mathrm{WB})$, & Santa Cruz & $\mathrm{sc}-542$ \\
& $1: 100(\mathrm{IHC})$ & & \\
$\alpha$ Lamin B & $1: 200(\mathrm{WB})$ & Santa Cruz & $\mathrm{sc}-6216$ \\
$\alpha$ GAPDH & $1: 25000(\mathrm{WB})$ & Millipore & MAB374 \\
$\alpha$ GFP & $1: 1000(\mathrm{WB})$ & Cell Signaling & $\# 2555$ \\
Goat anti-rabbit HRP & $1: 10,000(\mathrm{WB})$ & Santa Cruz & $\mathrm{sc}-2004$ \\
Goat anti-mouse HRP & $1: 10,000(\mathrm{WB})$ & Jackson & $115-035-062$ \\
& & ImmunoResearch & \\
Rabbit anti-goat HRP Fc & $1: 10,000(\mathrm{WB})$ & Jackson & $305-036-008$ \\
Fragment specific & & ImmunoResearch & \\
Goat anti-rabbit Alexa & $1: 100(\mathrm{IHC})$ & Invitrogen & A21429 \\
Flour 555 & & & \\
$\begin{array}{l}\text { Donkey anti-rabbit Texas } \\
\text { Red }\end{array}$ & $1: 50(\mathrm{IHC})$ & Jackson & $711-075-152$ \\
\hline
\end{tabular}


determined by the NanoDrop $1000 \mathrm{UV}-\mathrm{V}$ is spectrophotometer (Thermo Scientific). The cDNA synthesis was performed using the Superscript VILO cDNA synthesis kit (Invitrogen). Real time PCR was performed using TaqMan primer probe sets (Table 2-4) and TaqMan Fast Universal PCR Master Mix, No AmpErase UNG (Applied Biosystems). A cDNA template equivalent of 10ng was used for each reaction. The amplification was carried out in the Step One plus Real-Time PCR System (Applied Biosystems).

\section{Affymetrix array hybridization}

RNA purity and concentration was determined by using the NanoDrop 1000 UV-Vis spectrophotometer (Thermo Scientific). The integrity of RNA was determined by electrophoresis analysis using Agilent 2100 BioAnalyzer Lab on a Chip System (Agilent Technologies, Santa Clara, CA). The total RNA was processed by the Hartwell Center Microarray Core by using the Affymetrix Eukaryote one-cycle target labeling protocol ( $1 \mu \mathrm{g}$ of total RNA) or the 3' IVT Express (100 ng of total RNA).The RNA was first reverse transcribed using oligo dT primers containing $\mathrm{T} 7$ promoter followed by second strand synthesis. The resulting cDNA was in vitro transcribed with biotnylated ribonucleotides to generate the cRNA. The cRNA was hybridized to the 430v2 GeneChip array at $45^{\circ} \mathrm{C}$ overnight. The arrays were then washed and stained with streptavidin -PE. The arrays were scanned and an image file was produced. The expression values were summarized using the MAS5 algorithm as implemented in the GCOS v1.4 software (Affymetrix, Santa Clara, CA).

\section{Total protein extraction using Laemelli buffer}

One million cells were spun down and washed once with $1 \mathrm{ml}$ of PBS. The cell pellet was then resuspended in $100 \mu \mathrm{l}$ of ice cold PBS containing protease inhibitor cocktail (Sigma-Aldrich P8340) and AeBSF (Gold Biotechnology) at a 100× dilution. To the cell suspension $100 \mu 1$ of $2 \times$ Laemmeli sample buffer (Bio Rad Laboratories) containing beta mercaptoethanol $(20 \times)$ was added and then boiled at $100^{\circ} \mathrm{C}$ for 5 minutes. The samples were then stored in $-80^{\circ} \mathrm{C}$ before performing the immunoblot.

Table 2-4. TaqMan primer probe sets used for RT-PCR.

\begin{tabular}{ll}
\hline Gene Name & Catalogue Number \\
\hline mouse + human GATA2 & Mm00492302_g1 \\
mouse GATA2 & Mm00492300_m1 \\
mouse mycN & Mm00627179_m1 \\
mouse hoxA9 & Mm00439364_m1 \\
mouse GAPDH & Mm9999915_g1 \\
\hline
\end{tabular}

The primer probe sets were purchased from Applied Biosystems. 


\section{Cell compartment extraction using the Qproteome cell compartment kit}

The Qproteome cell compartment kit was purchased from Qiagen (Cat No 37502). Five million cells were spun down at $500 \mathrm{~g}$ for $10 \mathrm{~min}$ at $4{ }^{\circ} \mathrm{C}$ and washed twice with ice cold PBS. Protease inhibitors and AeBSF $(100 \times)$ were added to the buffers (CE1, CE2 and CE3). The cell pellet was resuspended in $250 \mu 1$ of buffer CE1 and incubated for 10 minutes at $4^{\circ} \mathrm{C}$ in an end over shaker. After a $1000 \mathrm{~g}$ spin for 10 minutes the supernatant containing the "cytoplasmic fraction " was extracted. The pellet was again resuspended in $250 \mu \mathrm{l}$ of buffer CE2 and incubated for 30 minutes at $4^{\circ} \mathrm{C}$ in an end over shaker. After a $6000 \mathrm{~g}$ spin for 10 minutes the supernatant containing the "membrane fraction " was extracted. Seven $\mu 1$ of benzonase was added to the pellet and incubated for 15 minutes at room temperature followed by $250 \mu 1$ of buffer CE3 for 10 minutes at $4{ }^{\circ} \mathrm{C}$ in an end over shaker. After a $6800 \mathrm{~g}$ spin for 10 minutes the supernatant containing the "nuclear fraction" was extracted. All the fractions were then stored in $80^{\circ} \mathrm{C}$ before performing the immunoblot.

\section{Immunoblot}

I added $2 \times$ Laemelli buffer containing beta-mercaptoethanol to the protein extracts and boiled for 5 minutes at $100^{\circ} \mathrm{C}$. The samples were loaded into NUPAGE 4-12 \% Bis-Tris gel (Invitrogen) and run at 200V for 1 hour: 30 min with NUPAGE MOPS SDS Running buffer. The gel was then transferred to PVDF membrane using NUPAGE transfer buffer at $30 \mathrm{~V}$ for 1 hour $30 \mathrm{~min}$. The membrane was blocked with $5 \%$ milk in TBS $0.1 \%$ - Tween 20 for 1 hour. The primary antibody was incubated overnight at $4^{\circ} \mathrm{C}$. After 3 washes with TBS-T the secondary antibody was incubated for 1 hour at room temperature. After 3 washes with TBS-T the blot is developed using ECL prime western blotting detection reagent (Amersham). Antibodies used and their dilutions are shown in Table 2-3.

\section{Electrophoretic mobility shift assay}

Human wild type and mutant GATA2 and GATA2-ERT proteins were synthesized from pCDNA3.1 expression plasmids using the Rabbit reticulocyte TnT kit (Promega). Four hundred thousand counts per minute of $\mathrm{P}^{32}$ labeled double stranded oligonucleotide containing the alpha globin promoter

(5'-GATCTCCGGCAACTGATAAGGATTCCCTG-3') were incubated with the protein lysates in the presence or absence of 500 molar excess of unlabeled double stranded oligonucleotides. Binding reactions contained $10 \mathrm{mM}$ Tris, $\mathrm{pH} 8.0,40 \mathrm{mM} \mathrm{KCl}, 0.05 \%$ NP-40, 6\% glycerol, $1 \mathrm{mM}$ DTT, $0.2 \mathrm{~g}$ poly dI/dC, $200 \mu \mathrm{M} \mathrm{ZnCl} 2$ and $5 \mu \mathrm{l}$ of synthesized GATA2 and GATA2-ERT lysates. Samples were incubated at $4{ }^{\circ} \mathrm{C}$ overnight and resolved by electrophoresis through a nondenaturing $4 \%$ polyacrylamide gel in 0.5 TBE (45 mM Tris-borate, $1 \mathrm{mM}$ EDTA) at room temperature and analyzed using the Storm 860 phosphoimager (Molecular Dynamics, Sunnyvale, California). 


\section{T4 kinase labeling reaction and annealing reactions}

The alpha-globin reverse oligo nucleotides were $5^{\prime}$ end labeled using the T4 Polynucleotide kinase kit (Promega M4101). The labeled oligos were purified using Illustra TM ProbeQuant G-50 Micro Columns (GE Healthcare 28-9034-08). The Reverse and forward oligonucleotides were annealed in $50 \mathrm{mM} \mathrm{MgCl} 2$ and $100 \mathrm{mM}$ Tris $\mathrm{pH} 8.0$ by boiling for 10 minutes followed by slow cooling to Room temperature overnight.

\section{TnT reactions}

In vitro transcription and translation was performed using the TnT T7 quick coupled transcription/translation system (Promega \# L1170). Two small scale reactions containing $2 \mu \mathrm{g}$ of the pCNDA3.1 constructs in a $50 \mu 1$ reaction was setup with either the cold methionine or $\mathrm{S}^{35}$ labeled methionine. The $\mathrm{S}^{35}$ labeled protein lysates was run on a 4-12 \% Bis-Tris gel (Invitrogen) to verify the presence of the GATA2 and GATA2-ERT proteins. The unlabeled protein lysates were used in the EMSA assay.

\section{Chromatin immunoprecipitation}

GATA2-ERT cells were harvested and stained with Gr-1-PE antibody and enriched for Gr-1 negative population by FACS sorting. $20 \times 10^{6}$ cells were crosslinked for 10 minutes by adding formaldehyde to a final concentration of $1 \%$. The reaction was quenched by adding glycine to a final concentration of $0.125 \mathrm{M}$ for 5 minutes. The fixed cells were washed twice with ice cold PBS and the cell pellet was snap frozen in dry ice with protease inhibitors and AeBSF. The cell pellet was later thawed and resuspended in $2 \mathrm{ml}$ of cell lysis buffer (10 mM Tris $\mathrm{pH} 8.0,10 \mathrm{mM} \mathrm{NaCl}, 0.2 \% \mathrm{NP}-40$ in water) with protease inhibitors and AeBSF for 10 minutes on ice. After a spin of $2500 \mathrm{rpm}$ for 10 minutes the nuclei pellet is resuspended in $800 \mu$ of Nuclei Lysis Buffer $(50 \mathrm{mM}$ Tris pH 8.0, $10 \mathrm{mM}$ EDTA, 1\% SDS in water) and sonicated. The sonication was done with 1.5 $\mathrm{ml}$ microfuge tubes containing $200 \mu \mathrm{l}$ aliquots in Bioruptor UCD-200 (Diagenode) for 30 cycles (30s "ON" and 30s "OFF" at 200W). This resulted in a shearing size between 200 bp to $700 \mathrm{bp}$. The sheared chromatin was diluted with $5 \times$ IP dilution buffer $(20 \mathrm{mM}$ Tris pH 8.0, 2 mM EDTA, $150 \mathrm{mM} \mathrm{NaCl}, 1 \%$ Triton X-100, 0.01\% SDS in water) and spun down at $14000 \mathrm{rpm}$ for five minutes to remove any undissolved nuclear material. Two $\mu \mathrm{g}$ of a rabbit polyclonal GATA2 antibody (sc-9008, Santa Cruz) or isotype matched preimmune IgG (ab37415, Abcam) was added to each $200 \mu 1$ of chromatin and incubated overnight at $4^{\circ} \mathrm{C}$ in a rotator platform. No antibodies were added to a $200 \mu \mathrm{l}$ aliquot which was used as input control. The chromatin was incubated for an additional 4 hours with $20 \mu \mathrm{l}$ of Protein A Dynabeads (\#100.01D Invitrogen). The beads containing immune-bound chromatin were collected by placing the microfuge tubes on a magnetic stand. The beads were washed three times with wash buffer ( 1 part nuclei lysis buffer +4 parts IP dilution buffer) and then resuspended with $200 \mu \mathrm{l}$ of elution buffer ( $100 \mathrm{mM}$ $\mathrm{NaHCO} 3,1 \%$ SDS in water). Reverse crosslink was performed at $65^{\circ} \mathrm{C}$ overnight in the presence of Proteinase K. Finally the supernatant containing immunoprecipitated DNA 
was pooled from several reactions and further purified using QIAquick PCR Purification Kit (\#28104, Qiagen) according to the kit protocols. Quantitative Real Time PCR is performed using SYBR green method with primers indicated on Table 2-5. 
Table 2-5. Primers used in quantitative chromatin immunoprecipitation assay.

\begin{tabular}{lll}
\hline Locus Name & Forward Sequence & Reverse Sequence \\
\hline a-globin promoter & TGACCAAGGTAGGAGGATACTAACTT & TTGCCCGGACACACTTCTTAC \\
Gata2 $(-) 1.8 \mathrm{~kb}$ & GCATGGCCCTGGTAATAGCA & CAGCCGCACCTTCCCTAA \\
Gata2 $(-) 2.8 \mathrm{~kb}$ & GCCCTGTACAACCCCATTCTC & TTGTTCCCGGCGAAGATAAT \\
Gata2 $(-) 3.9 \mathrm{~kb}$ & GAGATGAGCTAATCCCGCTGTA & AAGGCTGTATTTTCCAGGCC \\
Gata2 1 G promoter & AGATACCCAGAAGGTGCACGTC & GCAGACCCTGCACCCCT \\
Gata2 $1 \mathrm{~S}$ promoter & CCCCTCGAAGTGATGTCGAA & TCTGGCTGTCTCTCGGTTCC \\
necdin & GGTCCTGCTCTGATCCGAAG & GGGTCGCTCAGGTCCTTACTT \\
& & \\
mycN $-2.2 \mathrm{~kb}$ & ACACCTGTGCTCTTCACT & AGGTCCAAGGAAGATAAACGA \\
mycN $-9.8 \mathrm{~kb}$ & CACCCTGGCCCTTAGAAG & AGAGCAGAGAAGAAAGGGAAAG \\
mycN $+.6 \mathrm{~kb}$ & GCAGCCGTGACCTACATT & CGCGGGGAAAGAAGGAAAA \\
mycN $+2.3 \mathrm{~kb}$ & GTGTTTCCAGCCTCTTCTGT & TGCTGCGTTCCCTTTGTT \\
mycN $+3 \mathrm{~kb}$ & CACAGCCCCATGGAAAAAAA & CTACCACTGCCGCTCCTAA \\
mycN $+3.2 \mathrm{~kb}$ & AAGTGCAGGCTTATCACCAA & CCAGCCCCCCTATTAGAC \\
mycN $6.5 \mathrm{~kb}$ & GCGTGTCTGGCATTTGAG & CCCTGCCCAGATGATTCT \\
HoxA9 $-3.8 \mathrm{~kb}$ & GTGGTGGACAGGATAAAATGGA & GCTGTGCGTGGAAAAAGA \\
HoxA9 $-1.2 \mathrm{~kb}$ & AATGGCAGAGAAGAGTTGGAG & GCACTGCCTGAGACCACA \\
HoxA9 $-15 \mathrm{bp}$ & AACCGCTCTGGTATCCTT & GGAGGGAGGGGAGTAACAA \\
HoxA9 $+2 \mathrm{~kb}$ & CGGGTCTCCGCTCTACTC & ATGGGTGCCTCTGGATCT \\
HoxA9 $+3.8 \mathrm{~kb}$ & GCATCCCGGAGAACTATCT & GGAGAGAAGCTGTGGCATAA \\
HoxA $+7.5 \mathrm{~kb}$ & AGCCCAGACCCAGTTTT & ACAGGAAACGGAGGAAACAA \\
HoxA9 $+8.5 \mathrm{~kb}$ & AAACCCGACTTCACAATGGA & AGCCTGGACCCACTGAAA \\
HoxA9 $+5.6 \mathrm{~kb}$ control & GCTCACACAAATCCCATTCCA & CTTTCTCGCCGCCATCTC \\
& & \\
\hline
\end{tabular}




\section{CHAPTER 3. RESULTS}

\section{Downregulation of GATA2 Expression during Hematopoiesis in the Adult BM}

To monitor GATA2 expression during hematopoiesis we first isolated murine hematopoietic stem cells and various progenitor cells based on cell surface marker expression by FACS Sorting. The $\mathrm{Kit}^{+} \mathrm{Scal}^{+} \mathrm{Lin}^{-} \mathrm{CD} 150^{+} \mathrm{CD} 48^{-}$population (SLAM) is highly enriched for Long Term HSCs (LT-HSCs) in which one in 2.1 cells (47\%) yield long-term multilineage reconstitution in irradiated mice. ${ }^{129} \mathrm{The} \mathrm{Kit}^{+} \mathrm{Scal}^{+} \mathrm{Lin}^{-}$(LSK) population is a heterogeneous population of both short term reconstituting (ST-HSCs), long term reconstituting HSCs (LT-HSCs) and multipotent progenitors (MPPs). The $\mathrm{Kit}^{+}$ Scal ${ }^{-}$Lin $^{-}$population is devoid of stem cell activity and can be fractionated into Common Myeloid Progenitor (CMP), Granulocyte Monocyte Progenitor (GMP) and Megakaryocyte Erythroid Progenitor (MEP). ${ }^{130}$ The Common Lymphoid Progenitor (CLP) is present in the $\mathrm{Kit}^{\text {low }} \mathrm{Sca}^{\text {low }} \mathrm{Lin}^{-} \mathrm{CD} 127^{+}$population. ${ }^{131} \mathrm{BM}$ isolated from 10 C57BL/6 mice was pooled and sorted into the populations described above and lysed for RNA (Figure 3-1A). Based on quantitative RT-PCR analysis we found that GATA2 expression was highest in the LT-HSCs (Figure 3-1B). The heterogeneous LSK population had 1.5 fold lower expression than the LT-HSCs due to the presence of lower expressing ST-HSCs (Figure 3-1B). In the progenitor compartment GATA2 expression was downregulated more in the CLP (12 fold) than the myeloid progenitors (2.4-3.3 fold) (Figure 3-1B). The least expression was seen in the mature Lin $^{+}$cells (20 fold) (Figure 3-1B). In summary downregulation of GATA2 expression is seen during hematopoietic differentiation and myeloid progenitors have higher GATA2 expression than the lymphoid progenitors.

\section{GATA2-ERT Expressing BM Cells Exhibit TAM Independent GATA2 Function in CFU-C Assays}

Previous studies from our lab have demonstrated that bone marrow progenitors and stem cells subjected to enforced expression of GATA2 from a retroviral vector show a complete loss of colony forming activity in vitro and a loss of repopulating ability in $v i v o^{95}$. We developed an inducible GATA2-ERT vector in which the protein would be cytosolic in the absence of Tamoxifen (TAM) and with the addition of TAM enters into the nucleus thus allowing regulated GATA2 activity in response to Tamoxifen (Figure 3-2A). The GATA2-ERT vector was first tested for TAM-induced reduction in colony forming activity. Bone marrow cells transduced with the MSCV GATA2 ires GFP and MSCV GATA2-ERT ires GFP vectors showed similar GATA2 expression at the mRNA level compared to the control MSCV ires GFP vector transduced and untransduced BM cells (Figure 3-2B). The transduced BM cells were then plated in methylcellulose media for colony assays in the presence or absence of $100 \mathrm{nM}$ Tamoxifen. Consistent with our previous results, the proportion of GFP + colonies formed in the GATA2 group was 8 fold lower than the control GFP group irrespective of the Tamoxifen treatment (Figure 3-3A). GATA2-ERT transduced BM cells in the absence of Tamoxifen formed colonies 
A

PANEL 1
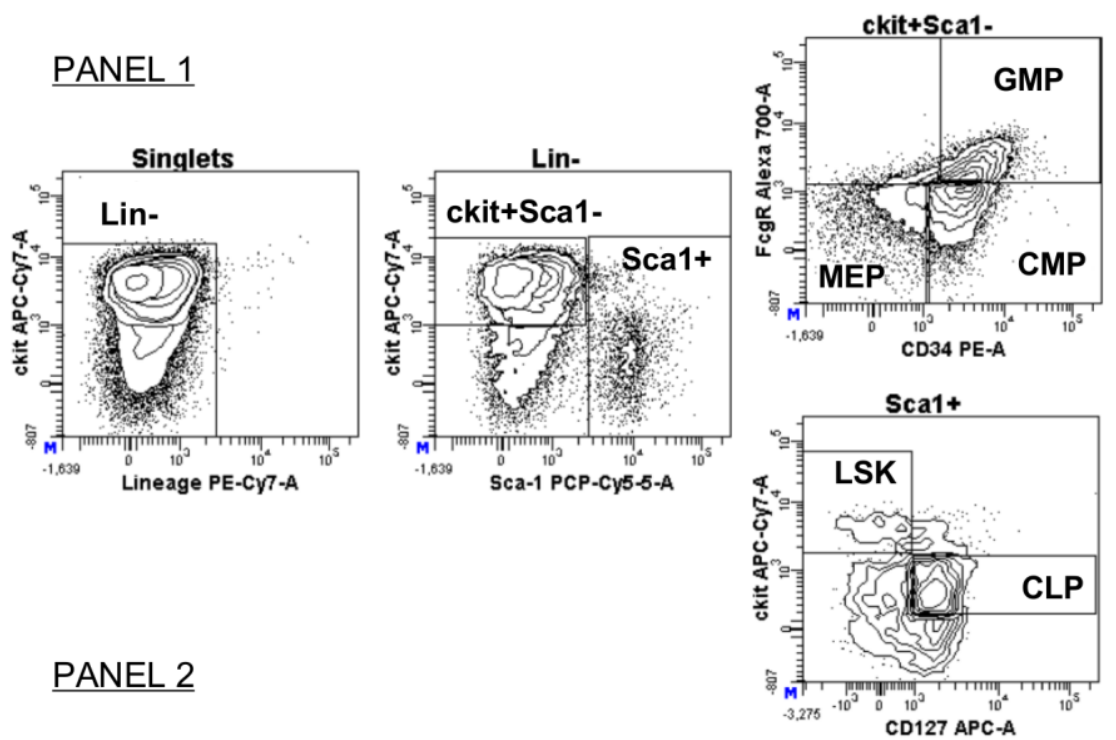

PANEL 2
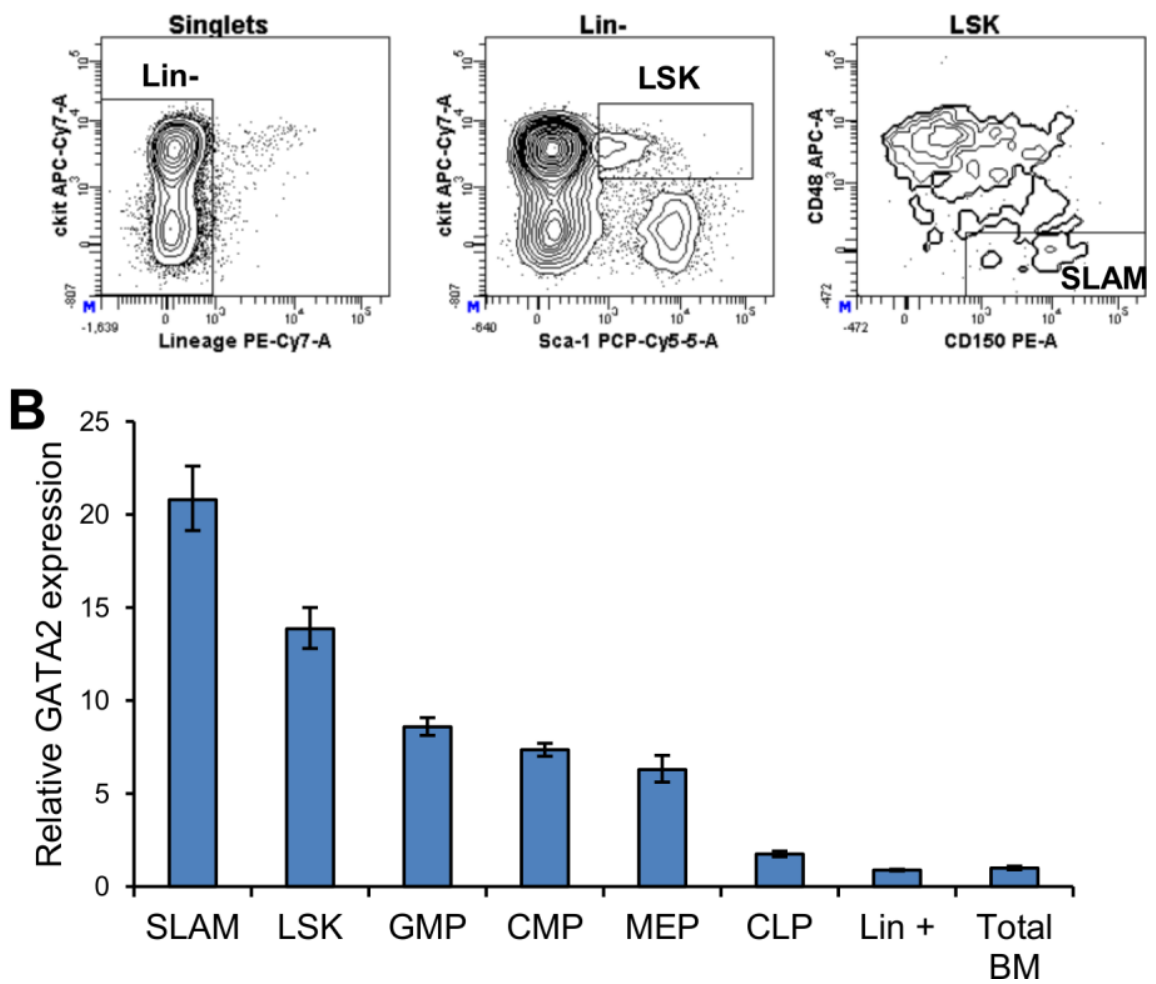

Figure 3-1. Endogenous GATA2 expression in various stem and progenitor compartments of the adult $B M$.

(A) Pooled BM from 10 mice is depleted of lineage markers (Ter119, Gr1, Mac1, CD4, CD8, B220, and NK1.1) and then stained for progenitor and stem cell markers (Panel 1CD34, ckit, Sca1, Fc $\gamma$ R and CD117; Panel 2 - ckit, Sca1, CD150, CD48). Detailed gating strategy used for sorting various compartments for subsequent RNA extraction is shown. (B) GATA2 expression levels in sorted stem and progenitor compartments relative to total BM using quantitative RT-PCR and TaqMan primer probe sets. GAPDH was used as the endogenous control. 
A MSCV-IRES-GFP

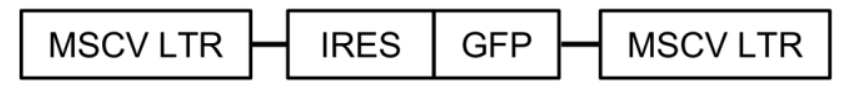

MSCV-GATA2-IRES-GFP

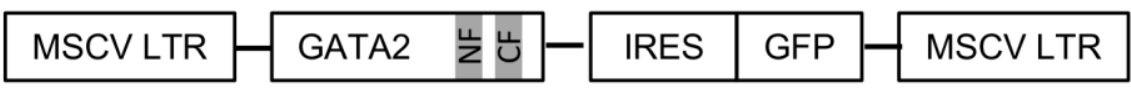

MSCV-GATA2-ERT-IRES-GFP

\begin{tabular}{|l|l|l|l|l|}
\hline MSCV LTR \\
\hline
\end{tabular} \begin{tabular}{|l|l|l|}
\hline GATA2 & 岁 & ERT \\
\hline
\end{tabular}

\begin{tabular}{|c|c|c|}
\multicolumn{1}{c}{ GATA2 } & \multicolumn{1}{c}{ Linker } & \multicolumn{1}{c}{ ERT } \\
\hline ...SSMVTAM & NSDPRNE & MGASGP... \\
\hline
\end{tabular}

B

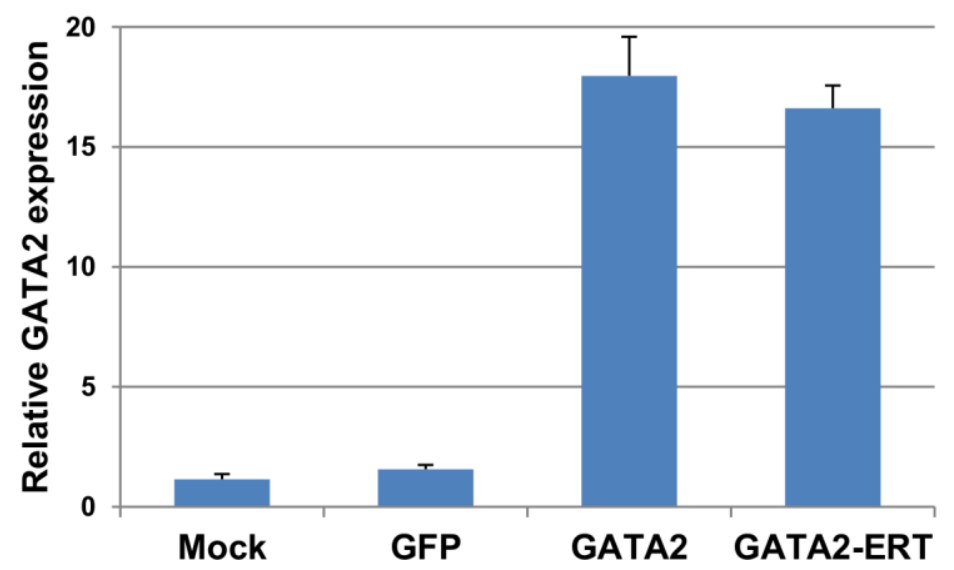

Figure 3-2. Successful transduction of murine BM cells with the inducible GATA2-ERT retroviral vector.

(A) Schematic of the MSCV based retroviral vectors used in the study (NF= N-terminus zinc finger, $\mathrm{CF}=\mathrm{C}$-terminus zinc finger). The vectors contain an internal ribosomal entry site (ires) that allows cap independent translation of the GFP coding sequence. Thus transgene GATA2-ERT dose in individual cells can be predicted based on GFP expression (Mean Fluorescence Intensity). The aminoacid sequences of the linker region connecting human GATA2 and the ERT in the MSCV GATA2-ERT vector is also shown. (B) Quantitative RT-PCR analysis of gene expression of total GATA2 (mouse \& human) in lineage depleted BM cells 2 days post-transduction with the above vectors. GAPDH was used as the endogenous control. 

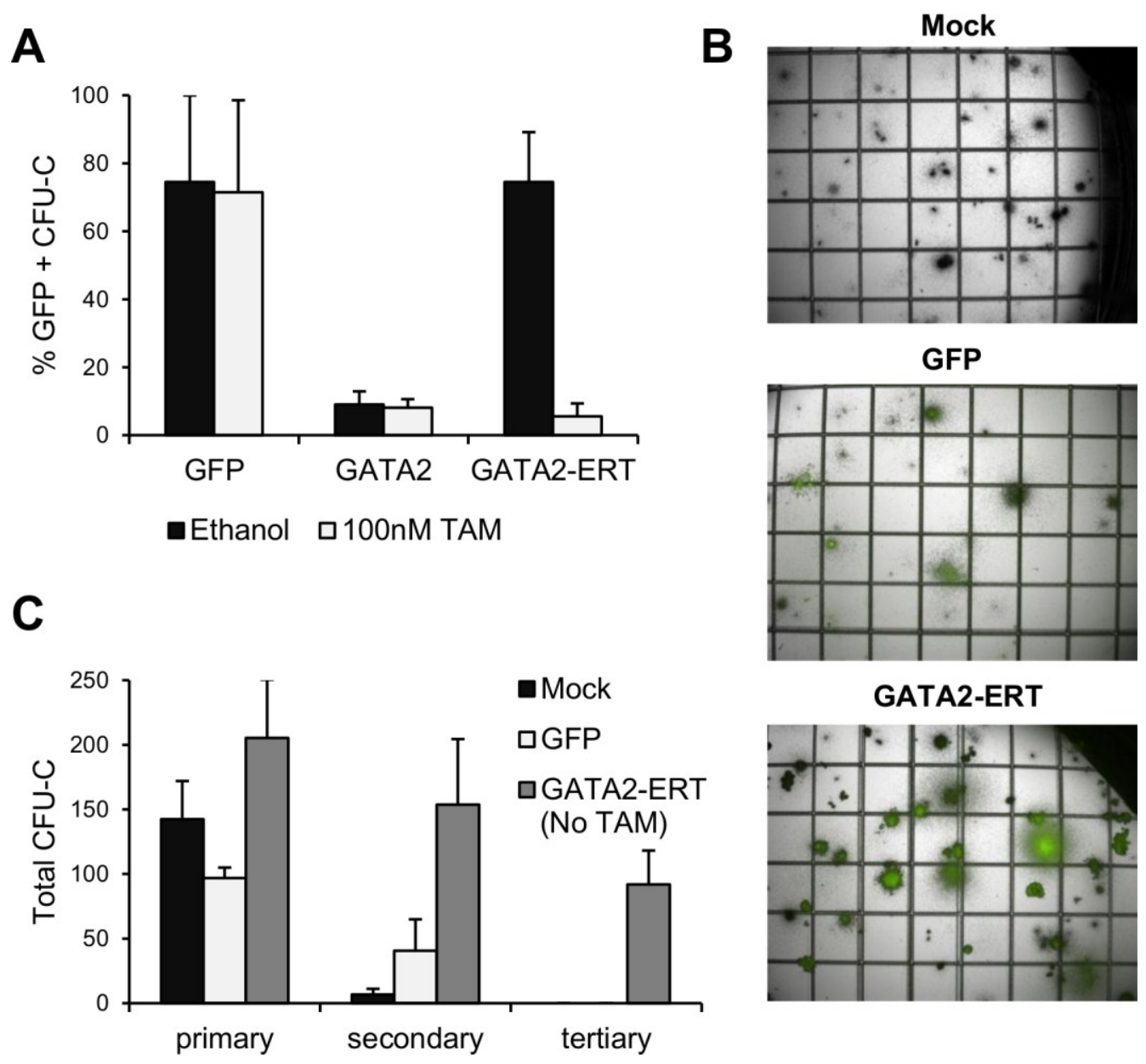

Figure 3-3. GATA2-ERT expressing cells exhibit TAM independent GATA2 function on myeloid CFU-C assays.

(A) Bone marrow cells transduced with MSCV-iresGFP, MSCV-GATA2iresGFP and MSCV-GATA2-ERTires GFP vectors were plated in semisolid media (Methocult M3434) in the presence of $100 \mathrm{nM}$ Tamoxifen or equivalent volume of Ethanol. 7 days later total and GFP + ve primary colonies were counted to calculate the percentage of GFP $+\mathrm{ve}$ CFU-C $(\mathrm{n}=3)$. (B) Representative images of primary colonies in the absence of Tamoxifen. (C) CFU-Cs from MSCViresGFP and GATA2-ERTiresGFP and mock transduced BM cells were tested for serial replating ability $(n=3)$. Total colonies were scored, harvested and re-plated every 7 days after plating. Cells were plated at 5 X103 per plate in the primary plating followed by $2.5 \mathrm{X} 104$ per plate for secondary and tertiary plating respectively. 
similar in number to the control GFP vector transduced cells. However, in the presence of $100 \mathrm{nM}$ Tamoxifen there was a 13-fold reduction in the GATA2-ERT colonies, an effect similar to the GATA2 vector. Surprisingly the sizes of the GATA2-ERT colonies in the absence of Tamoxifen were consistently larger than the mock and control GFP vector colonies (Figure 3-3B). To test if this larger colony size is associated with increased self-renewal activity we tested the GATA2-ERT vector against the control vector in a serial colony replating assay. Cells harvested from primary colonies of GATA2-ERT transduced cells were able to consistently generate four-fold higher secondary colonies than the GFP transduced cells (Figure 3-3C). When replated for tertiary colonies, only the GATA2-ERT transduced cells retained cells with colony forming, clonogenic potential.

\section{GATA2-ERT Vector Immortalizes Primary BM Cells into Myeloid Cell Lines}

Given the effect of GATA-2ERT expression on endowing serial replating potential on hematopoietic progenitors, we next tested whether expression would have effects on cells propagated in vitro liquid cultures. Transduced BM cells were cultured in DMEM with 15\% FBS in the presence of IL-3, IL-6 and SCF. Under these culture conditions normal untransduced BM cells cease to proliferate at 3-4 weeks becoming mast cells. ${ }^{132}$ GATA2-ERT transduced bone marrow cells showed greater proliferation compared to the GFP transduced and untransduced cells (Figure 3-4A) frequently resulting in the establishment of immortalized cell lines (6 out of 7 experiments). Wright Giemsa stains indicated that the GATA2-ERT immortalized cell lines were composed of immature myeloid cells with some spontaneous differentiation (Figure 3-4B). This was further confirmed by FACS analysis, which showed that the $50 \%$ of cells are Gr1+ Mac1+ in these cell lines compared to the controls which become mast cells expressing ckit+ FcER1+ (Figure 3-4C). Only once the control GFP vector immortalized BM cells ( 1 out of 7 experiments) resulting in a GFP cell line. All of these immortalized cell lines are IL-3 dependent (data not shown). Upon treatment with $100 \mathrm{nM}$ of Tamoxifen all the GATA2-ERT cell lines stopped proliferating (Figure 3-5A) similar to the CFU-C assays. Cell cycle analysis showed cell cycle arrest with increased G0/G1 phase as early as 24 hours post TAM treatment in GATA2-ERT cell lines (Figure 3-5B). Thus, in the presence of TAM, the GATA2-ERT vector behaves as a wild type GATA2.

\section{Low Nuclear Concentration Achieved by the GATA2-ERT Vector in the Absence of TAM}

The increased proliferation and colony forming ability of the GATA2-ERT vector in the absence of Tamoxifen could be the result of low levels of GATA2-ERT entering the nucleus which has been reported in other inducible vectors like mycER and creER. ${ }^{133,134}$ To test this hypothesis, subcellular protein fractionation was performed on untreated, Tamoxifen $(100 \mathrm{nM})$ and Ethanol (vehicle) treated GATA2-ERT cell lines. GATA2-ERT protein was detected in both the nuclear and cytoplasmic fractions of untreated and vehicle treated cells confirming the activity of the GATA2-ERT vector in 
A

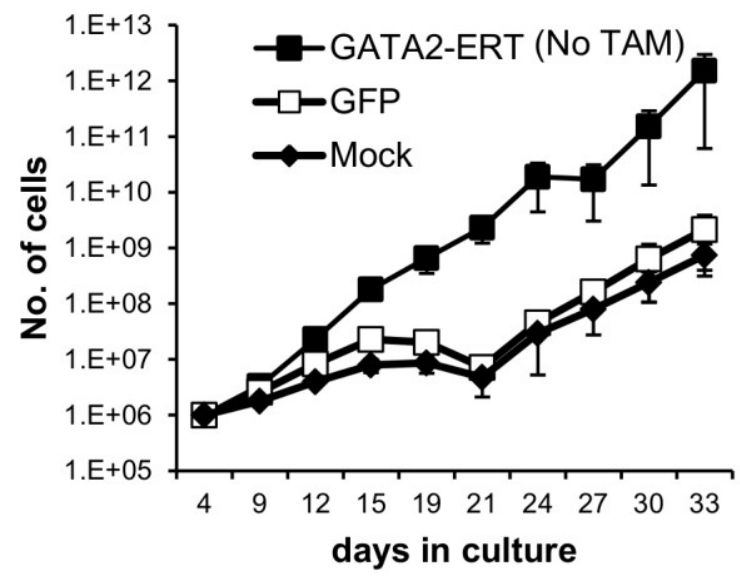

B

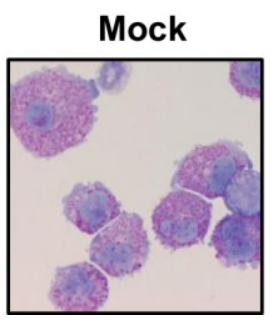

C
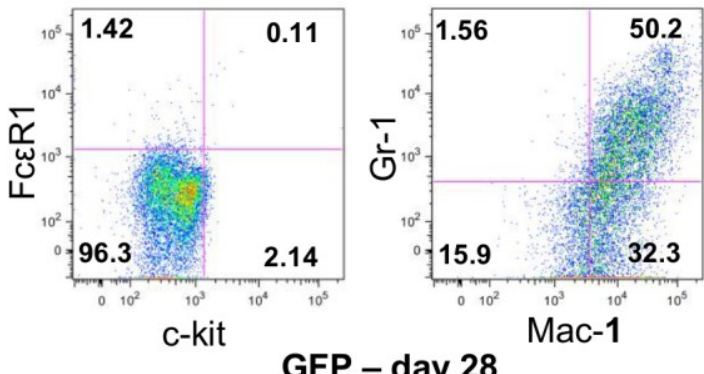

GFP - day 28
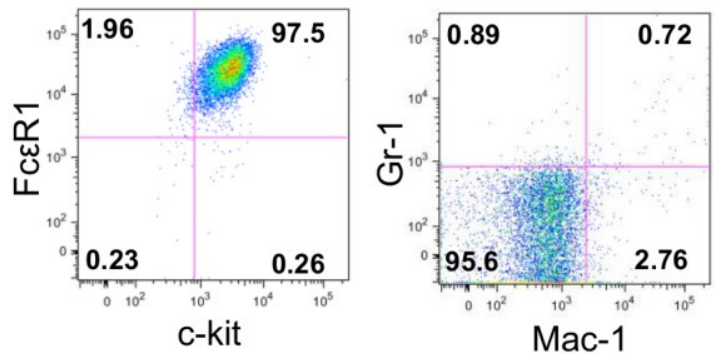

Figure 3-4. GATA2-ERT immortalizes primary BM cells into myeloid cell lines. (A) Growth curves of lineage depleted BM cells transduced with MSCV-GATA2ERTiresGFP and MSCV-iresGFP vector compared with untransduced cells. Cells were counted at the time points indicated by trypan blue exclusion method. Results are shown as mean \pm SD for 3 independent experiments. (B) Wright-Giemsa stained cytospin slides at day 27 under light microscopy. (C) FACS analysis at day 28 after staining for mast cell markers (c-kit, FceRI) and myeloid markers (Gr-1, Mac-1). 
A

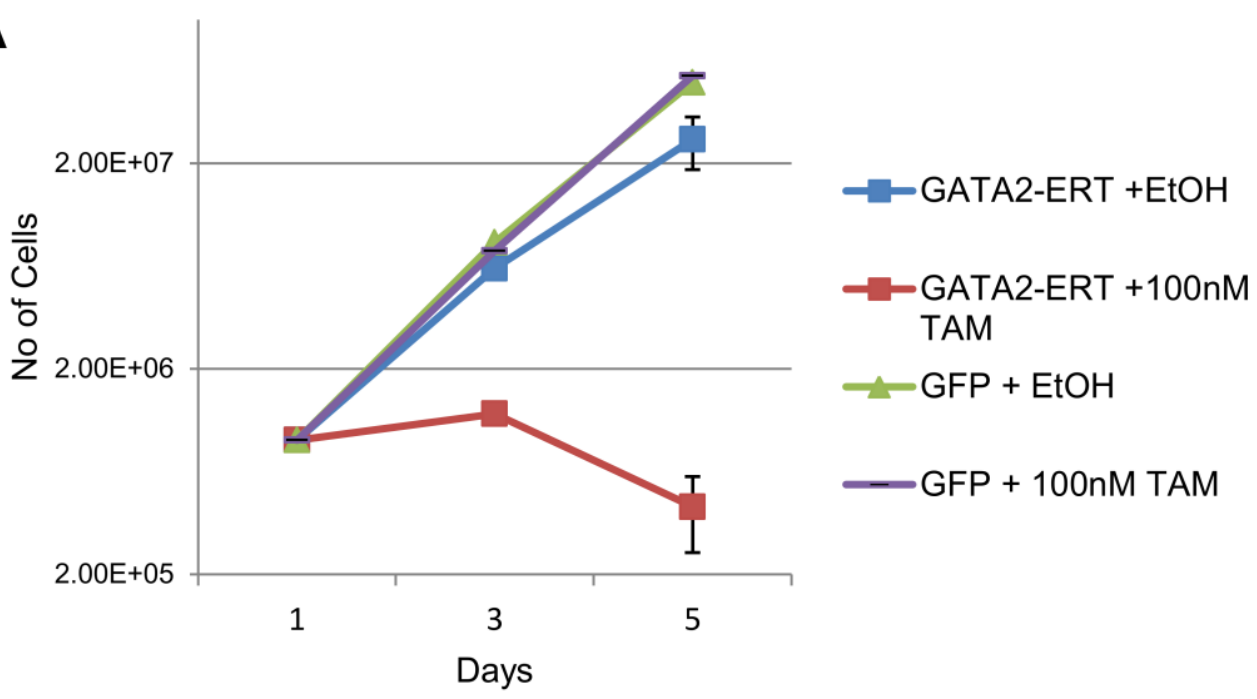

B

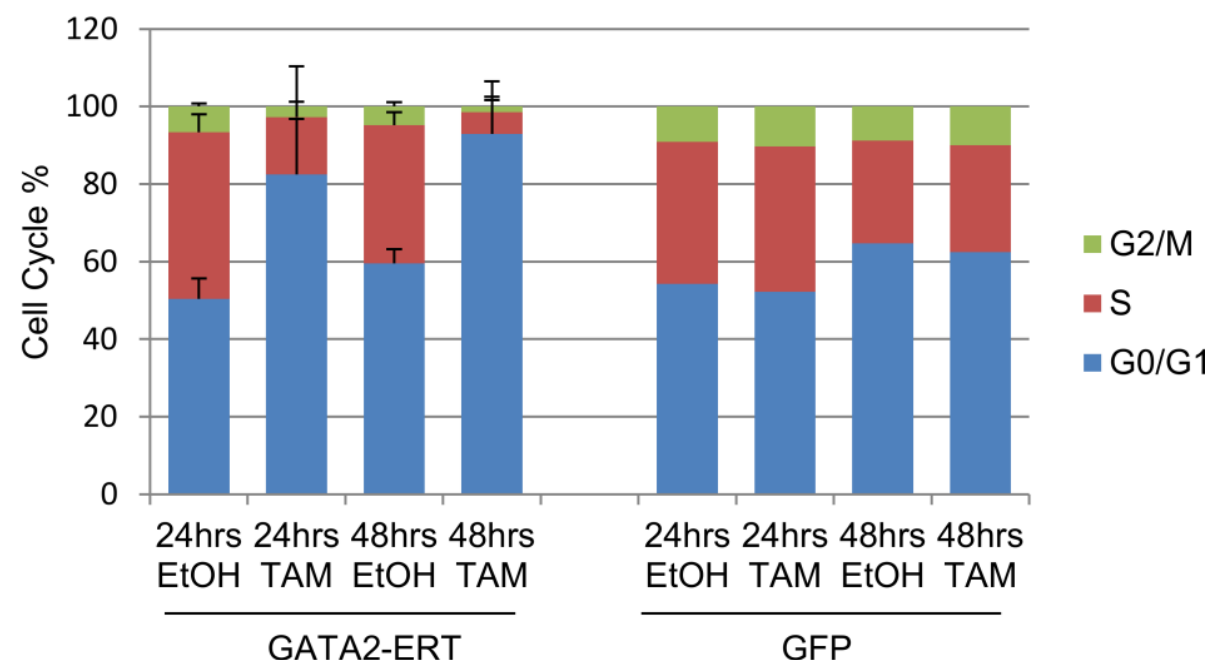

Figure 3-5. Effects of the absence and presence of Tamoxifen on GATA2-ERT cell lines.

(A) Growth curves of GATA2-ERT and GFP cell lines treated with $100 \mathrm{nM}$ Tamoxifen (TAM) or an equivalent volume of Ethanol (EtOH). (B) Cell cycle analysis of GATA2ERT and GFP cell lines treated with $100 \mathrm{nM}$ TAM or EtOH. At 24 and 48 hours posttreatment cells are lysed with Propidium Iodide and analyzed by flow cytometry. Results are shown as mean $\pm \mathrm{SD}$ of experiments on 3 different GATA2-ERT cell lines and 1 GFP cell line. 
the absence of Tamoxifen (Figure 3-6A). Upon treatment with $100 \mathrm{nM}$ Tamoxifen, GATA2-ERT protein was detected only in the nuclear fractions showing complete nuclear translocation. Thus GATA2-ERT translocation in the absence of Tamoxifen could achieve a low level tonic activity in the nucleus, 3-fold lower than with complete translocation with Tamoxifen as measured by densitometry analysis using ImageJ software. To independently confirm these results we also did immunostaining on untreated and TAM treated GATA2-ERT cell lines (Figure 3-6B) and retroviral packaging cells (Figure 3-7) followed by confocal imaging. In the absence of TAM both these cells showed nuclear GATA2-ERT protein staining which co-localized with DAPI. Upon TAM treatment, the intensity of the nuclear GATA2-ERT signals increased demonstrating further nuclear translocation. The cytoplasmic GATA2-ERT signals in the absence of TAM could only be visualized in the packaging cells but not in the GATA2ERT cell lines possibly due to the presence of thin cytoplasm in these cells. These data confirm the low nuclear concentration achieved by the GATA2-ERT vector in the absence of TAM.

\section{Distinct Progenitor and Mature Cell Populations Are Present in GATA2-ERT Cell Lines}

When GATA2-ERT cell lines and the randomly immortalized GFP cell lines were stained for c-kit and Gr-1 markers, three different populations were consistently observed (Figure 3-8A). These populations were named P1 (c-kit+Gr-1- ), P2 (c-kit-Gr-1- ) and P3 $(\mathrm{Gr}-1+)$ respectively. Upon Wright- Giemsa staining P1 and P2 populations showed an immature phenotype with large nucleus and thin cytoplasm whereas the P3 population showed mature granulocytes with segmented nuclei (Figure 3-8B). When these populations were sorted from GATA2-ERT cell lines and then cultured for 7 days, the highest proliferation was observed in $\mathrm{P} 1$ followed by $\mathrm{P} 2$ and the lowest proliferation seen in P3 (Figure 3-8C) . In 7 days sorted P1 cells can generate P2 and P3, P2 cells can generate only P3 and finally P3 cells did not generate other populations (Figure 3-8A, D and $\mathbf{E}$ ). Taken together, the above data show that GATA2-ERT cell lines are organized as a hierarchy with the progenitor $\mathrm{P} 1$ cells differentiating into an intermediate $\mathrm{P} 2$ cells and finally becoming the mature P3 cells.

\section{Myeloid Immortalization Is GATA2-ERT Dependent}

To confirm if the GATA2-ERT immortalized cell lines are indeed dependent upon the low level tonic GATA2 activity we examined the effects of GATA2-ERT knockdown in three independently immortalized GATA2-ERT cell lines. We used a mir30 based shRNA containing lentiviral vectors carrying m-cherry as a reporter (Figure 3-9A). Because a single mRNA transcript is generated from the MSCV GATA2-ERT ires GFP vector for the production of both GATA2-ERT and GFP proteins we tested GATA2-ERT knockdown using a shRNA targeting GFP (shGFP). After transduction with shGFP vector, mcherry + ve transduced cells showed an 80\% GATA2-ERT protein knockdown compared to mcherry -ve untransduced cells (Figure 3-9B). The shGFP 


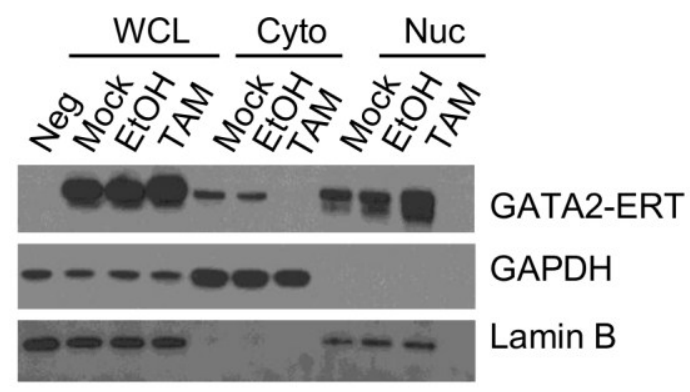

B

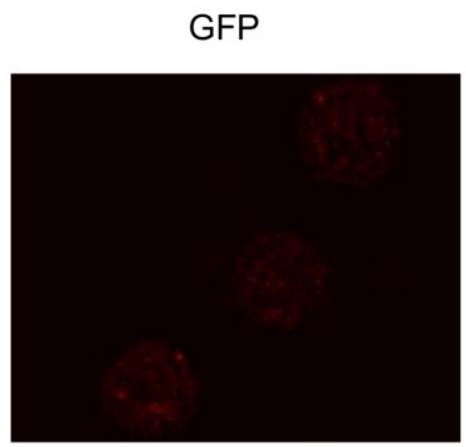

$$
\text { GATA2-ERT + EtOH }
$$

GATA2-ERT + 100nM TAM
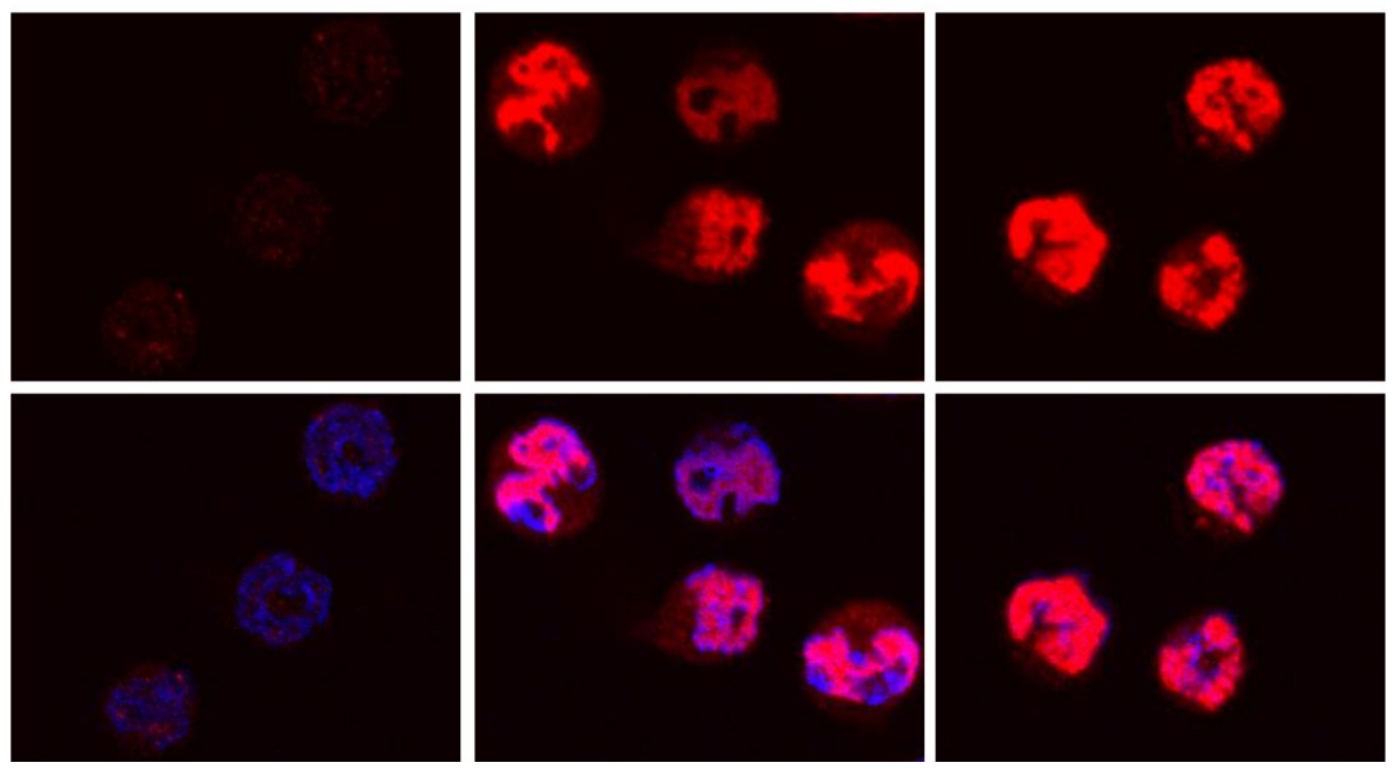

Figure 3-6. Subcellular localization of GATA2-ERT before and after treatment with TAM in GATA2-ERT immortalized cell lines.

(A) Subcellular localization of GATA2-ERT protein in GATA2-ERT cell lines after treatment with $100 \mathrm{nM}$ TAM or ethanol for 3 hours compared with untreated cells. Proteins from GATA2-ERT cell lines (WCL) were sub-fractionated into cytoplasmic (Cyto) and nuclear (Nuc) and western blotted for GATA2-ERT, GAPDH (cyto) and Lamin B (Nuc). GATA2-ERT protein was detected using an anti-ER antibody. (B) GATA2-ERT and GFP cell lines were attached retronectin coated coverslips in the presence of $100 \mathrm{nM}$ TAM or EtOH for 12 hours then fixed and stained. Red represents GATA2-ERT $\left(1^{\circ}-\alpha\right.$ GATA2, $2^{\circ}-\alpha$-rabbit Alexa Flour 555) and blue represents DAPI. Top row shows GATA2-ERT staining and bottom row shows an overlay with DAPI. Imaging was performed using Zeiss LSM 510 Meta point scanning confocal microscope with a Plan Apochromat 63X/1.4 Oil objective and zoom scan of 1.3. 


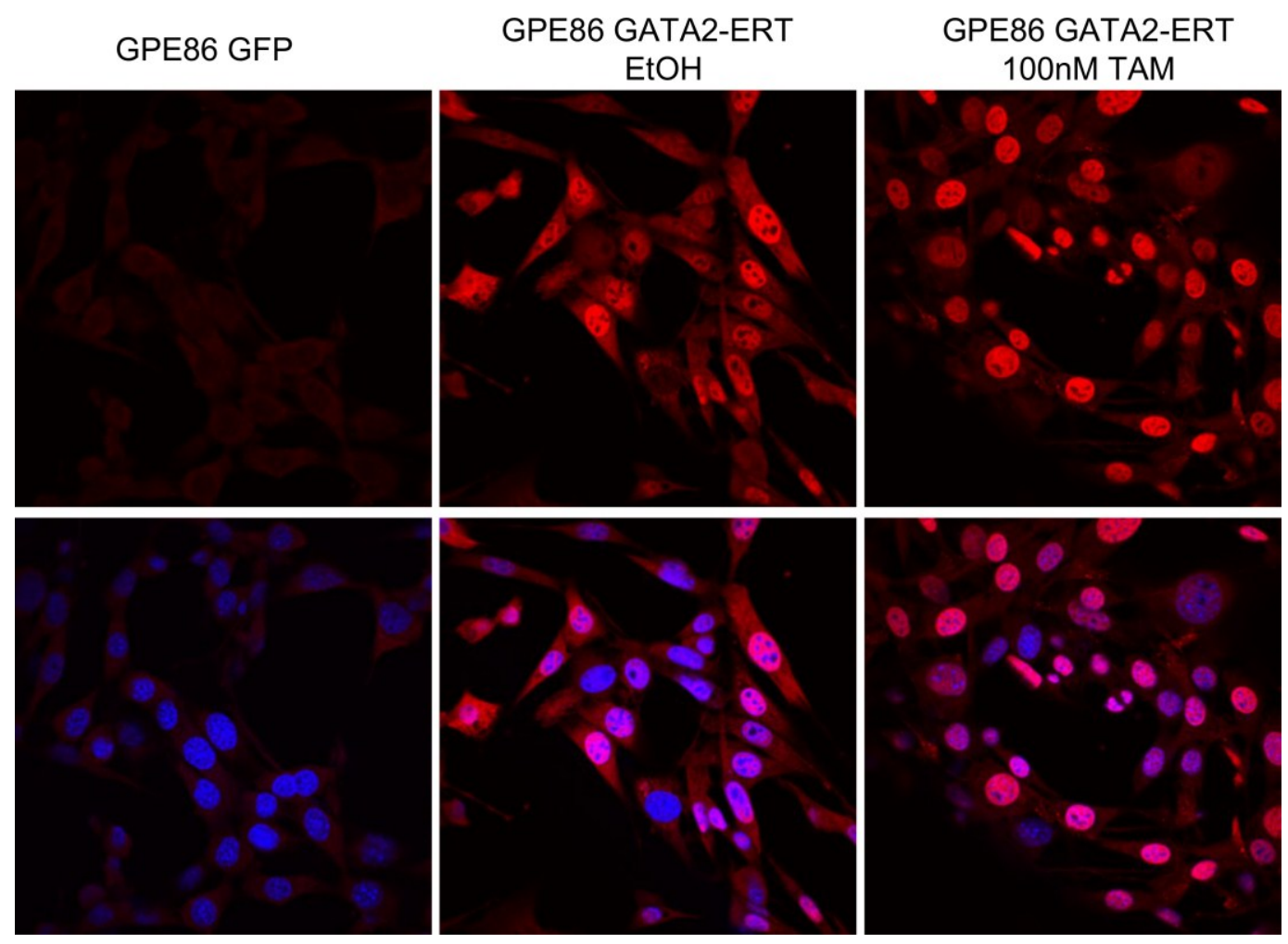

Figure 3-7. Subcellular localization of GATA2-ERT in GPE-86 producer cells. GPE-86 GFP and GATA2-ERT cells were attached to Poly-D lysine coated glass bottom dishes overnight and then treated with $100 \mathrm{nM}$ TAM/Ethanol for 24 hours before fix and stain. Red represents GATA2-ERT ( $1^{\circ}$ - $\alpha$ GATA2, $2^{\circ}-\alpha$-rabbit Texas Red $)$ and blue represents DAPI. Top row shows GATA2-ERT staining and bottom row shows an overlay with DAPI. Imaging was performed using Nikon C1Si laser scanning confocal microscope with a Plan Apochromat 60.0X/1.45/.13 Oil objective. 


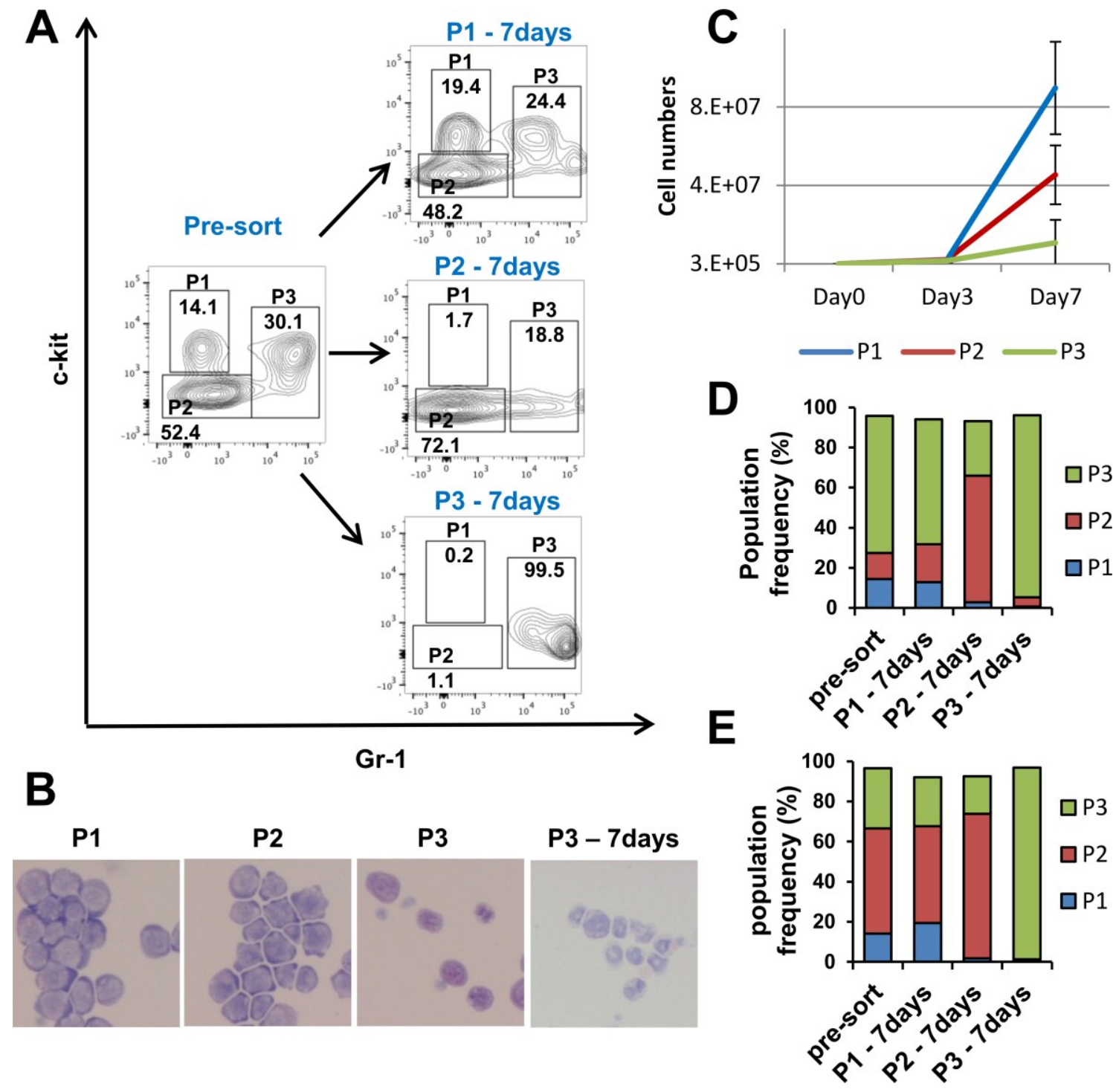

Figure 3-8. Distinct progenitor and mature cell populations are present in GATA2-ERT cell lines.

(A) Representative FACS Analysis of unsorted GATA2-ERT cell line and sorted P1, P2 and $\mathrm{P} 3$ populations after 7 days in culture, showing the ability of the sorted populations to regenerate the starting cell line. (B) Wright Giemsa stains on cytopsins of sorted P1, P2, $\mathrm{P} 3$ populations and $\mathrm{P} 3$ population after 7 days in culture. (C) Growth curves of the sorted populations during the 7 days in culture. (D) Data from Figure 3-8A represented as bar graphs comparing the frequencies of different populations. (E) Data from a second experiment performed on another independently generated GATA2-ERT cell line showing the existence of a hierarchy. 
A

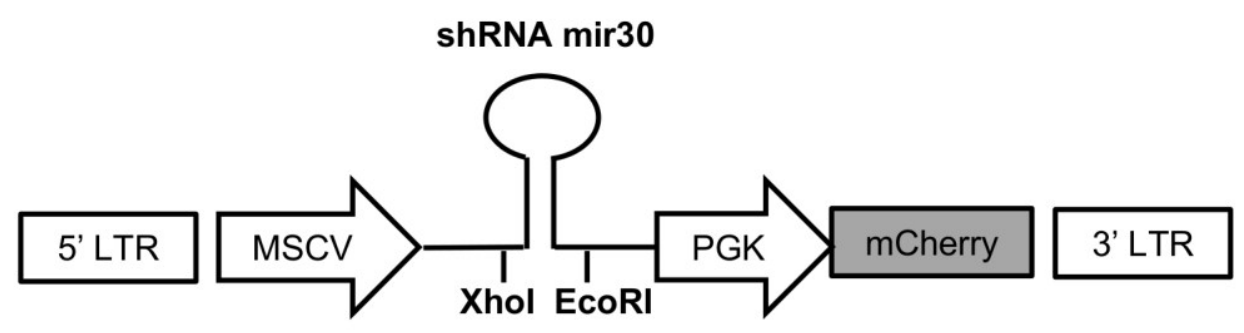

B

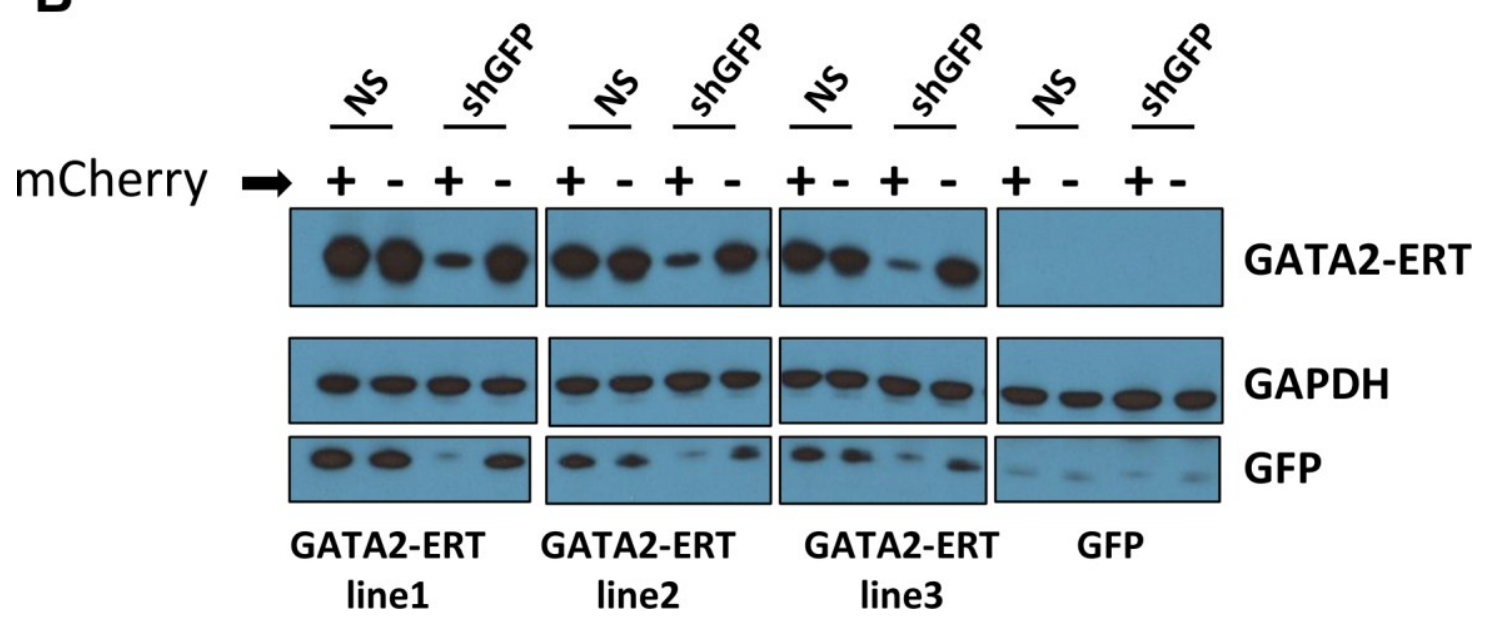

Figure 3-9. GFP targeting vector effectively knocks down GATA2-ERT protein levels.

(A) Schematic of the mir30 based lentiviral shRNA vector used in the study. MSCV promoter drives the mir30 based shRNA and PGK promoter drives the reporter mcherry expression. Location of the EcoRI and XhoI sites used for cloning 137bp hairpins is also shown. (B) GFP targeting vector effectively knocks down GATA2-ERT protein levels. Three GATA2-ERT cell lines and a GFP cell line were transduced with shRNA vectors targeting GFP sequence (shGFP) or a scrambled sequence (NS). 3 days post-transduction mcherry + and mcherry - cells were sorted and lysed for proteins and western blotted against GATA2-ERT ( $\alpha$-ER alpha), GAPDH and GFP. 
vector transduced cells grew faster than untransduced cells in liquid culture as shown by a progressive reduction in $\mathrm{m}$-cherry $\%$ in the shGFP group over an 18 day period (Figure 3-10A and B). On the other hand when GATA2-ERT cells were transduced with the control non silencing vector (NS) both transduced and untransduced cells showed a similar GATA2-ERT protein expression (Figure 3-9B) and equal proliferation in liquid culture with a stable m-cherry\% over an 18 day period (Figure 3-10A and B). When the GFP cell line with similar myeloid characteristics to the GATA2-ERT cells was transduced with the shGFP and NS vectors there were no changes in proliferation excluding other toxic effects of microRNA expression in myeloid cells (Figure 3-10A and B). To identify the nature of the growth defect associated with GATA2-ERT knockdown we looked at the cell cycle, cell death and differentiation of the GATA2-ERT cell lines after transduction with the shGFP vector. There was no increase in dead cells in these cultures based on trypan blue staining (data not shown). However cell cycle analysis using Hoechst staining showed a decrease in cycling cells after GATA2-ERT knockdown (Figure 3-11). This also correlated with the increase in P3 differentiated cells in these cultures (Figure 3-12A, B and C). These data demonstrate that expression of GATA2-ERT remains essential for self-renewal and proliferation of the myeloid progenitors after immortalization and loss of GATA2-ERT leads to their rapid differentiation into non-cycling, Gr1 expressing, mature cells.

\section{Screening of Genes Involved in GATA2-ERT Mediated Immortalization}

Gene expression analysis was performed to identify genes differentially regulated between three independently generated GATA2-ERT cells lines compared to the GFP cell line which does not express GATA2. A total of 1861 probesets showed a greater than 2 -fold difference between each of the GATA2-ERT cell lines vs. the GFP cell line. Among them, 995 probesets were increased and 866 probesets were decreased. Estrogen receptor1 (Esr1) probeset was on the top of the list with a 500 fold average fold difference. Since the human GATA2 cDNA is fused to the estrogen receptor ligand binding domain in the vector we expected to see high expression of the Esr1 probeset. Gene ontology and pathway enrichment analysis was performed using DAVID bioinformatics databases. Differentially regulated genes were grouped into genes regulating cell proliferation (143 probsets, Table 3-1), hematopoiesis (57 probesets) and apoptosis (100 probestes). Top 10 upregulated and downregulated genes in these lists are shown in Tables 3-1, 3-2 and 3-3. Myeloid leukemia related genes like HoxA9, Nmyc and Bcll la were found to be significantly up-regulated in the microarray. Real time PCR analysis using TaqMan primers confirmed that Nmyc was 50-fold up regulated and HoxA9 was 2400-fold up regulated in the GATA2-ERT cell lines (Figure 3-13A).

\section{HoxA9 and Nmyc Knockdown Partially Recapitulates GATA2-ERT Loss in Immortalized Cell Lines}

The knockdown approach described above was used to test if HoxA9 or Nmyc is required for GATA2-ERT mediated proliferation. The vectors designed for knockdown 

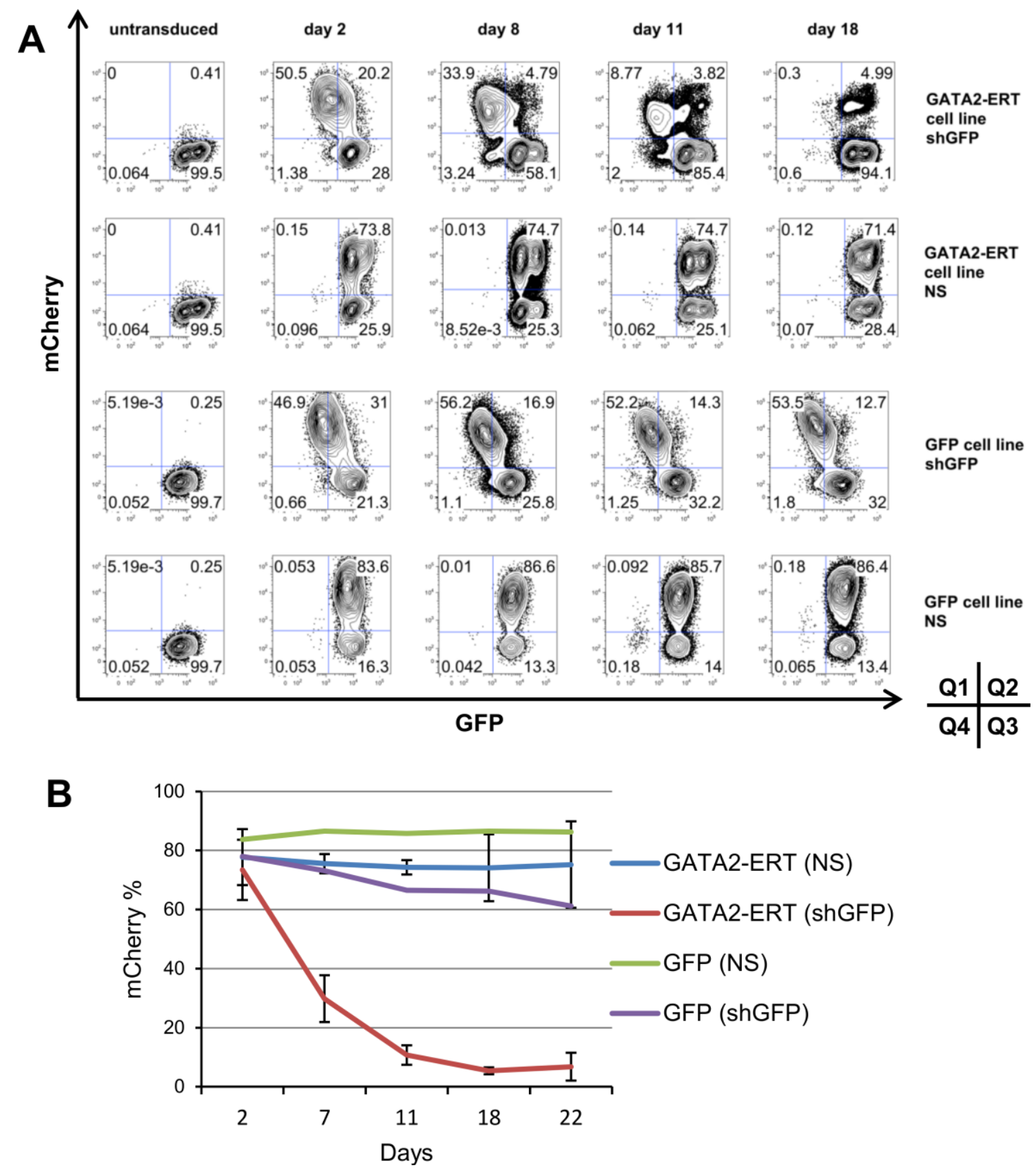

Figure 3-10. Knockdown of GATA2-ERT inhibits proliferation of the immortalized cell lines.

(A) GATA2-ERT knockdown inhibits proliferation of GATA2-ERT immortalized cell lines. FACS analysis of GFP and mcherry expression in GATA2-ERT and GFP cell lines transduced with shGFP and NS vectors at different time points in liquid culture. Cells were also stained with Hoechst for simultaneous cell cycle analysis. Transduction with shGFP vector results in a GFP low mcherry+ population (Quadrant 1) compared to GFP high mcherry+ (Quadrant 2) population in NS transduced cells. (B) Summary of the percentages of mcherry+ cells from above and 2 other GATA2-ERT cell lines. 


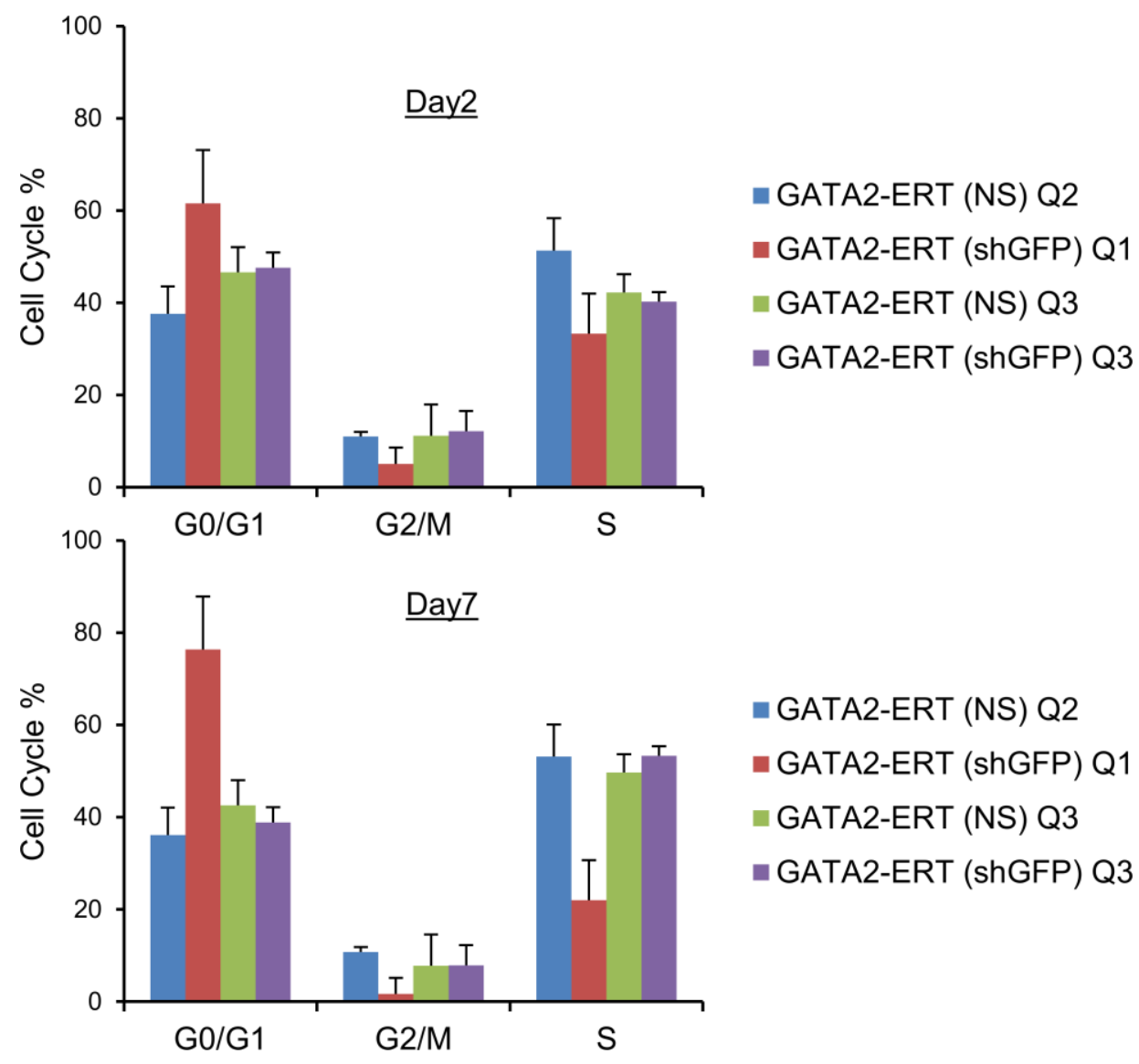

Figure 3-11. Knockdown of GATA2-ERT inhibits proliferation of the immortalized cell lines due to G0/G1 cell cycle arrest.

Cell cycle analysis using Hoechst was performed on GATA2-ERT cell lines $(n=3)$ at day 2 and day 7 post transduction with the NS and shGFP targeting vectors. Cell cycle effects on GATA2-ERT cells transduced with shGFP (quadrant 1, Q1) were compared with NS transduced cells (quadrant 2, Q2) and untransduced cells (quadrant 3, Q3) in both groups. The location of the quadrants was shown in Figure 3-10A. 

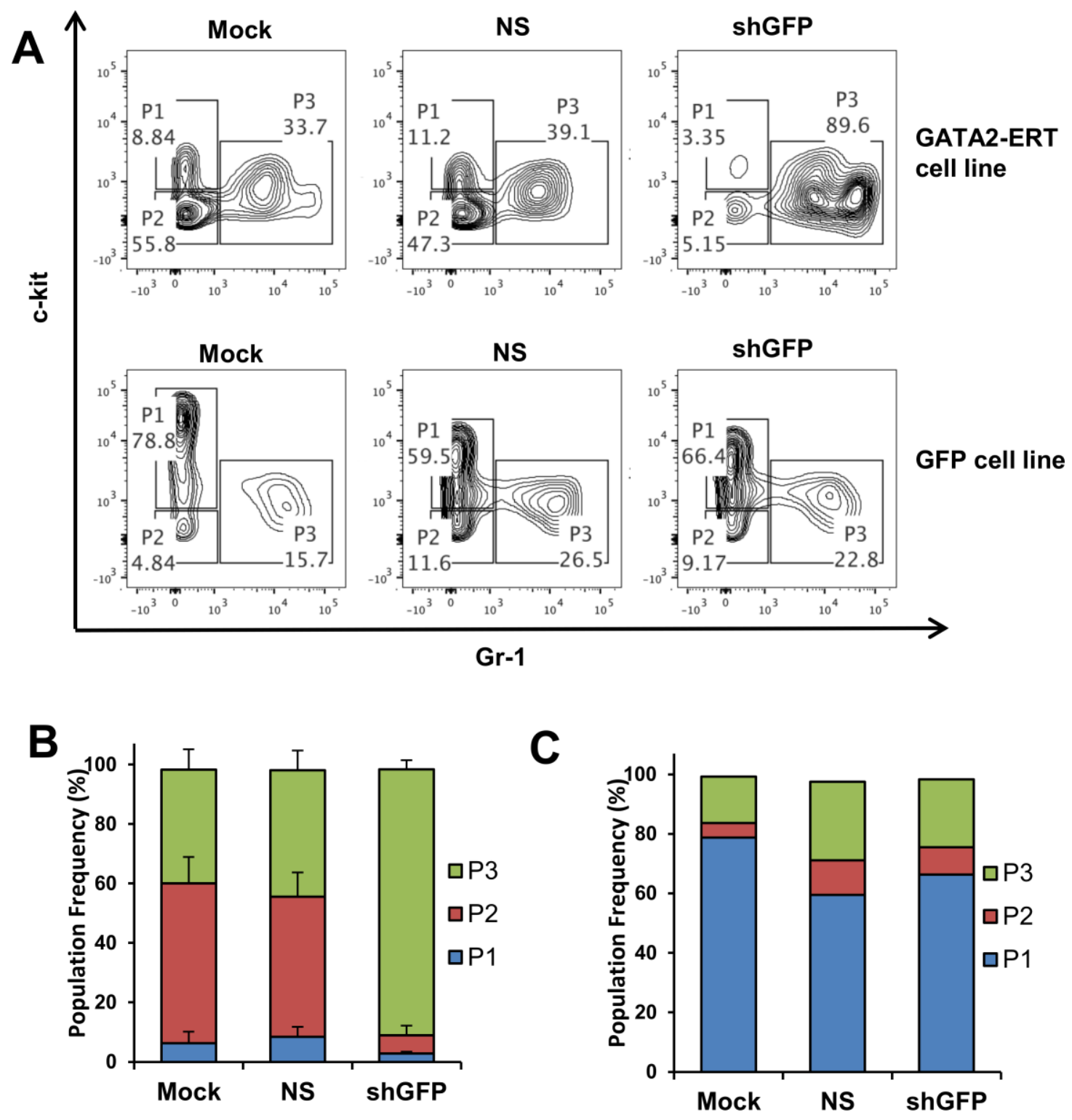

Figure 3-12. Knockdown of GATA2-ERT inhibits proliferation of the immortalized cell lines due to differentiation.

(A) Representative FACS Plots of GATA2-ERT and GFP cell lines transduced with the non-silencing (NS) and GFP silencing (shGFP) shRNA vectors and Mock transduced cells 6 days post- transduction. (B) Summary of the frequencies of different populations from above and 2 other GATA2-ERT cell lines $(n=3)$. (C) Frequencies of different populations from the above GFP cell line $(n=1)$. 
Table 3-1. Genes differentially expressed in GATA2-ERT vs. GFP cell line (cell proliferation).

\begin{tabular}{llc}
\hline Gene Symbol & Description & Fold Change \\
\hline Esr1 & estrogen receptor 1 (alpha) & 500.3 \\
Mycn & v-myc myelocytomatosis viral related oncogene, & \\
& neuroblastoma derived (avian) & 59.7 \\
Arrdc4 & arrestin domain containing 4 & 20.2 \\
Cd28 & CD28 antigen & 17.5 \\
Il6st & interleukin 6 signal transducer & 16.0 \\
S1pr1 & sphingosine-1-phosphate receptor 1 & 14.9 \\
Pawr & PRKC, apoptosis, WT1, regulator & 14.9 \\
Fgf6 & fibroblast growth factor 6 & 9.6 \\
Trim24 & tripartite motif-containing 24 & 9.2 \\
Sox2 & SRY-box containing gene 2 & 9.0 \\
Tnfrsf9 & tumor necrosis factor receptor superfamily, member 9 & -20.2 \\
Mab21l1 & mab-21-like 1 (C. elegans) & -21.1 \\
Il2ra & interleukin 2 receptor, alpha chain (probe 1) & -22.1 \\
Tnfsf10 & tumor necrosis factor (ligand) superfamily, member 10 & -26.0 \\
Hes1 & hairy and enhancer of split 1 (Drosophila) & -28.5 \\
Angptl2 & angiopoietin-like 2 & -35.1 \\
Fgf3 & fibroblast growth factor 3 & -40.3 \\
Arnt2 & aryl hydrocarbon receptor nuclear translocator 2 & -45.3 \\
Il2ra & interleukin 2 receptor, alpha chain (probe 2) & -104.0 \\
Mab21l1 & mab-21-like 1 (C. elegans) & -165.0 \\
\hline
\end{tabular}

Individual Fold changes of genes differentially expressed in three GATA2-ERT cell lines compared to the GFP cell line. Differentially expressed genes involved in regulation of proliferation (GO: 0042127) were shortlisted using DAVID bioinformatics databases (http://david.abcc.ncifcrf.gov). Top 10 differentially regulated genes are represented above. 
Table 3-2. Genes differentially expressed in GATA2-ERT vs. GFP cell line (hematopoiesis).

\begin{tabular}{llc}
\hline Gene Symbol & Description & Fold Change \\
\hline Hoxa9 & homeo box A9 & 77.0 \\
Bcl11a & B-cell CLL/lymphoma 11A (zinc finger protein) & 36.8 \\
Cd28 & CD28 antigen & 17.5 \\
Eomes & eomesodermin homolog (Xenopus laevis) & 11.3 \\
Hdac9 & histone deacetylase 9 & 11.1 \\
Clcf1 & cardiotrophin-like cytokine factor 1 & 8.4 \\
Ercc1 & Excision repair cross-complementing rodent repair & \\
& deficiency, complementation group 1 & 7.6 \\
Satb1 & special AT-rich sequence binding protein 1 & 7.3 \\
Pbx1 & pre B-cell leukemia transcription factor 1 & 7.3 \\
Tgfbr2 & transforming growth factor, beta receptor II & 5.8 \\
Themis & thymocyte selection associated & -6.8 \\
Card11 & caspase recruitment domain family, member 11 & -7.8 \\
Jag2 & jagged 2 & -7.8 \\
Gadd45g & growth arrest and DNA-damage-inducible 45 gamma & -8.4 \\
& X-ray repair complementing defective repair in & \\
Xrcc5 & Chinese hamster cells 5 & -11.8 \\
Kit & kit oncogene & -13.0 \\
Bcl6 & B-cell leukemia/lymphoma 6 & -14.9 \\
Cd74 & CD74 antigen (invariant polypeptide of major & \\
$H b b-b 1 / / /$ & histocompatibility complex, class II antigen-associated) & -17.5 \\
Hbb-b2 & hemoglobin, beta adult major chain /// hemoglobin, & -26.6 \\
$H b a-a 1 / / /$ & beta adult minor chain & -30.6 \\
$H b a-a 2$ & hemoglobin alpha, adult chain 1 /// hemoglobin alpha, & \\
\hline
\end{tabular}

Individual Fold changes of genes differentially expressed in three GATA2-ERT cell lines compared to the GFP cell line. Differentially expressed genes involved in regulation of hematopoiesis (GO: 0030097) were shortlisted using DAVID bioinformatics databases (http://david.abcc.ncifcrf.gov). Top 10 differentially regulated genes are represented above. 
Table 3-3. Genes differentially expressed in GATA2-ERT vs. GFP cell line (apoptosis).

\begin{tabular}{|c|c|c|}
\hline Gene Symbol & Description & Fold Change \\
\hline Esrl & estrogen receptor 1 (alpha) & 500.3 \\
\hline$C d 28$ & CD28 antigen & 17.5 \\
\hline Itsn 1 & intersectin 1 ( $\mathrm{SH} 3$ domain protein $1 \mathrm{~A})$ & 10.3 \\
\hline Uaca & $\begin{array}{l}\text { uveal autoantigen with coiled-coil domains and } \\
\text { ankyrin repeats }\end{array}$ & 9.2 \\
\hline $\mathrm{Clu} / / /$ & clusterin /// similar to clusterin & 8.0 \\
\hline \multicolumn{3}{|l|}{ LOC100046120 } \\
\hline Erccl & $\begin{array}{l}\text { Excision repair cross-complementing rodent repair } \\
\text { deficiency, complementation group } 1\end{array}$ & 7.6 \\
\hline $\operatorname{Cln} 8$ & ceroid-lipofuscinosis, neuronal 8 & 7.3 \\
\hline Kitl & kit ligand & 7.1 \\
\hline Epha7 & Eph receptor A7 & 6.6 \\
\hline Mgmt & O-6-methylguanine-DNA methyltransferase & 6.3 \\
\hline $\operatorname{Tn} f s f 9$ & tumor necrosis factor (ligand) superfamily, member 9 & -13.3 \\
\hline Tnfrsfg & tumor necrosis factor receptor superfamily, member 9 & -14.6 \\
\hline Bcl6 & B-cell leukemia/lymphoma 6 & -14.9 \\
\hline Btg2 & B-cell translocation gene 2, anti-proliferative & -14.9 \\
\hline$C d 74$ & $\begin{array}{l}\text { CD74 antigen (invariant polypeptide of major } \\
\text { histocompatibility complex, class II antigen- } \\
\text { associated) }\end{array}$ & -17.5 \\
\hline Tnfrsfg & tumor necrosis factor receptor superfamily, member 9 & -20.2 \\
\hline Tnfsf10 & $\begin{array}{l}\text { tumor necrosis factor (ligand) superfamily, member } \\
10\end{array}$ & -26.0 \\
\hline Prnp & prion protein & -33.5 \\
\hline Gzmb & granzyme B & -97.0 \\
\hline Il2ra & interleukin 2 receptor, alpha chain & -104.0 \\
\hline $\operatorname{Id} 3$ & inhibitor of DNA binding 3 & -143.7 \\
\hline
\end{tabular}

Individual Fold changes of genes differentially expressed in three GATA2-ERT cell lines compared to the GFP cell line. Differentially expressed genes involved in regulation of apoptosis (GO: 0042981) were shortlisted using DAVID bioinformatics databases (http://david.abcc.ncifcrf.gov). Top 10 differentially regulated genes are represented above. 

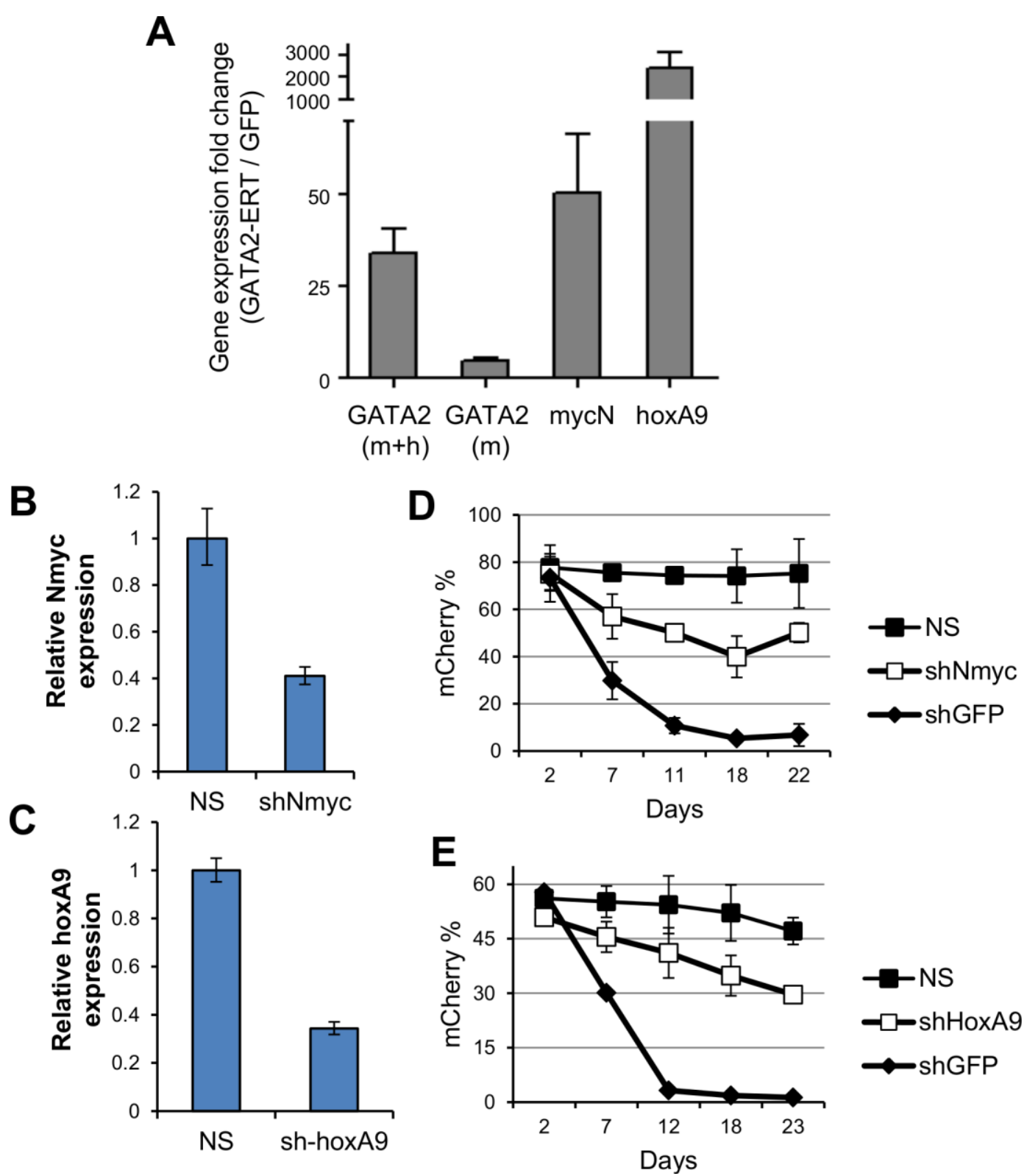

Figure 3-13. HoxA9 and Nmyc knockdown partially recapitulates GATA2-ERT loss in immortalized cell lines.

(A) Quantitative RT-PCR analysis of gene expression of Nmyc, hoxA9, endogenous (m) and total $(m+h)$ GATA2 in GATA2-ERT cell lines $(n=3)$ and GFP cell line $(n=1)$. Gene expression is first normalized to GAPDH and represented as a ratio between GATA2ERT and GFP cell lines. (B) and (C) GATA2-ERT cell line was transduced with shRNA vectors targeting Nmyc (shNmyc), hoxA9 (shHoxA9) and a scrambled sequence (NS). 3 days post-transduction mcherry + cells were sorted for total RNA extraction followed by quantitative RT-PCR. Expression levels of Nmyc and hoxA9 after knockdown relative to the scrambled vector is shown. GAPDH was used as the endogenous control. (D) and (E) FACS Analysis of m-cherry percentages of GATA2-ERT cell lines transduced with the respective shRNA vectors at different time points in liquid culture. 
of Nmyc and HoxA9 showed 60\% knockdown efficiency in the GATA2-ERT cell lines (Figure 3-13B and C). The well characterized shGFP and NS vectors were now used as controls. GATA2-ERT cell lines were transduced with the knockdown vectors at a similar MOI (50\%-80\% m-cherry + cells) and grown in liquid culture for 3 weeks. As expected GATA2-ERT cells transduced with the shGFP vector show a significant growth disadvantage compared to the non-silencing vector (NS) in liquid culture. Upon GATA2-ERT knockdown there is a progressive decrease in the m-cherry \% over the 18-day culture period (Figure 3-13D). During this time period the non-silencing vector transduced cells showed no significant changes in m-cherry + cells. Nmyc and HoxA9 knockdown also resulted in a progressive decrease in m-cherry+ cells but at slower rate than the shGFP group. The m-cherry marking in the shNmyc group dropped slowly from $75 \%$ on day 2 to $40 \%$ on day 18 compared to the shGFP group where it dropped quickly from $73 \%$ on day 2 to $10 \%$ on day 11 (Figure 3-13D). Similarly in another experiment the m-cherry marking in the shHoxA9 group dropped slowly from $50 \%$ on day 2 to $30 \%$ on day 21 compared to the shGFP group it dropped quickly from $57 \%$ on day 2 to $3 \%$ on day 12 (Figure 3-13E). These results show that Nmyc and HoxA9 at least have a partial role in GATA2-ERT mediated proliferation. The fact that the growth defects are not complete can be explained by the lack of complete Nmyc and HoxA9 knockdown by these vectors and also by compensation by other GATA2 targets.

\section{Absence of GATA2-ERT Chromatin Occupancy in the Nmyc and HoxA9 Locus}

Since Nmyc and HoxA9 have a partial role in GATA2-ERT mediated proliferation; chromatin immunoprecipitation (ChIP) assays were performed to test whether Nmyc and HoxA9 are direct targets of GATA2-ERT. Protein extraction from GATA2-ERT cell lines under various non-denaturing conditions with protease inhibitors resulted in the rapid proteolysis of the 83KD GATA2-ERT protein into smaller fragments between 27KD to 40KD detected by an ER specific antibody (Figure 3-14A). Only when the cells were lysed in denaturing conditions using Laemelli sample buffer could this degradation be prevented. Since the GATA2-ERT cell lines contain Gr1+ granulocytes it is possible that proteolytic enzymes in their granules could cleave GATA2-ERT protein during extraction. When the GATA2-ERT cells were sorted into Gr1+ and Gr1populations the total GATA2-ERT protein expression was similar in all the population under denaturing conditions (Figure 3-14B). When the proteins were extracted under non-denaturing conditions we could see less proteolysis in the Gr1- population indicating that the enzymes in the mature Gr1+ fraction cause the degradation (Figure 3-14B). The degradation is even further reduced in the Gr1- cells when the cells were fixed with formaldehyde and chromatin extracted (Figure 3-14C). So we extracted chromatin from Gr1- GATA2-ERT cells and performed ChIP assays using a rabbit polyclonal antibody against GATA2 (H-116 Santa Cruz) and normal rabbit immunoglobulins (IgG). We did quantitative PCR on the immunoprecipitated DNA fragments using primers that amplify known GATA2 binding regions in the GATA2 locus. The $-3.9 \mathrm{~kb},-1.8 \mathrm{~kb}$ and $-2.8 \mathrm{~kb}$ regions in the GATA2 locus were significantly enriched ( 25 fold, 9 fold and 6.5 fold) confirming that the H-116 antibody effectively immunoprecipitates the GATA2-ERT protein (Figure 3-15A). Inspection of the Nmyc and HoxA9 locus revealed several 


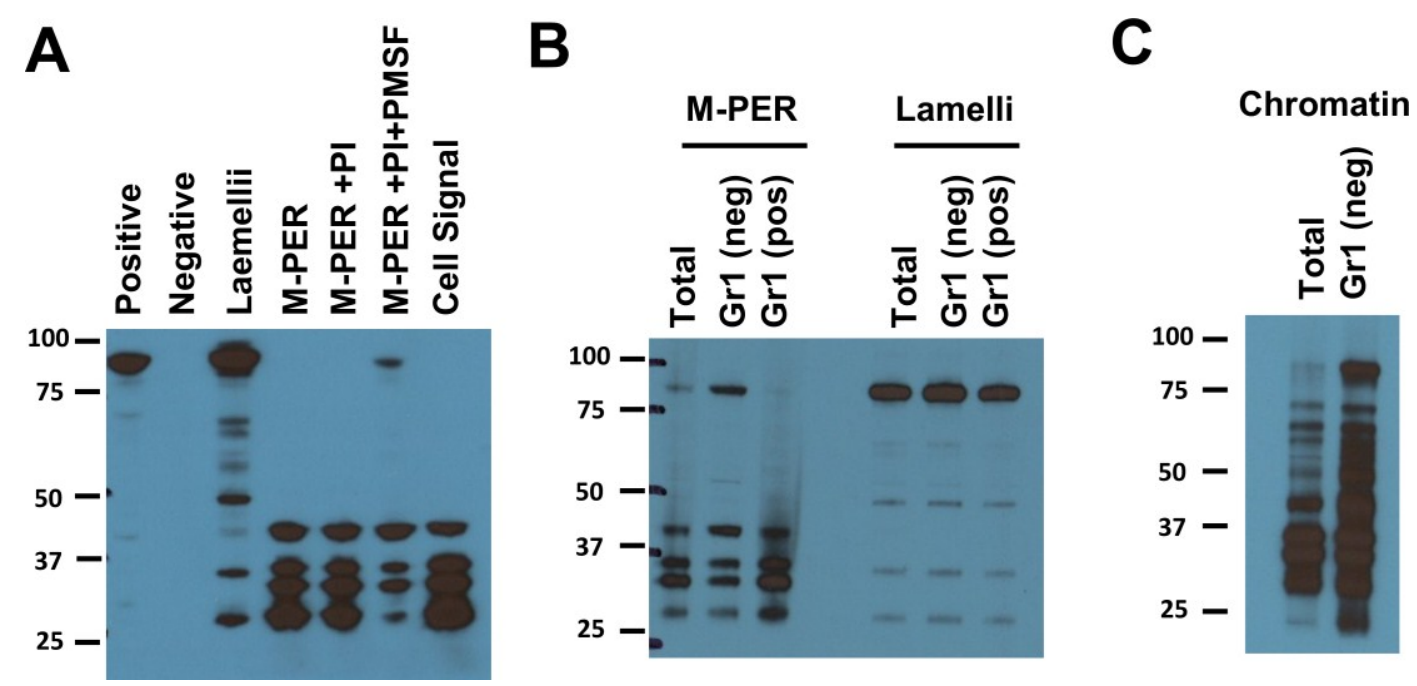

Figure 3-14. Elimination of the mature Gr1+ population in the GATA2-ERT cell lines prevents protein degradation and improves yield.

Western blots under denaturing conditions using anti-ER antibody to test GATA2-ERT protein degradation. Protein lysates were extracted from a G2ERT cell line under different conditions described. (A) Proteins were extracted from GATA2-ERT cell lines under non-denaturing conditions with reagents such as M-PER (Pierce \#78501) and Cell Lysis Buffer (Cell Signaling \# 9803) or denaturing conditions with Laemelli and betamercaptoethanol in the presence of protease inhibitors (PI) and PMSF. GPE-86 Producer cells expressing GATA2-ERT (positive) and GFP (negative) vectors were used as controls. (B) Proteins were extracted from unsorted and sorted Gr1 positive (Gr1 pos) and negative (Gr1 neg) populations under denaturing (Laemelli) and non-denaturing (MPER) conditions. (C) Fixed and sonicated chromatin samples were prepared from unsorted and Gr negative populations were western blotted after reverse cross link. 


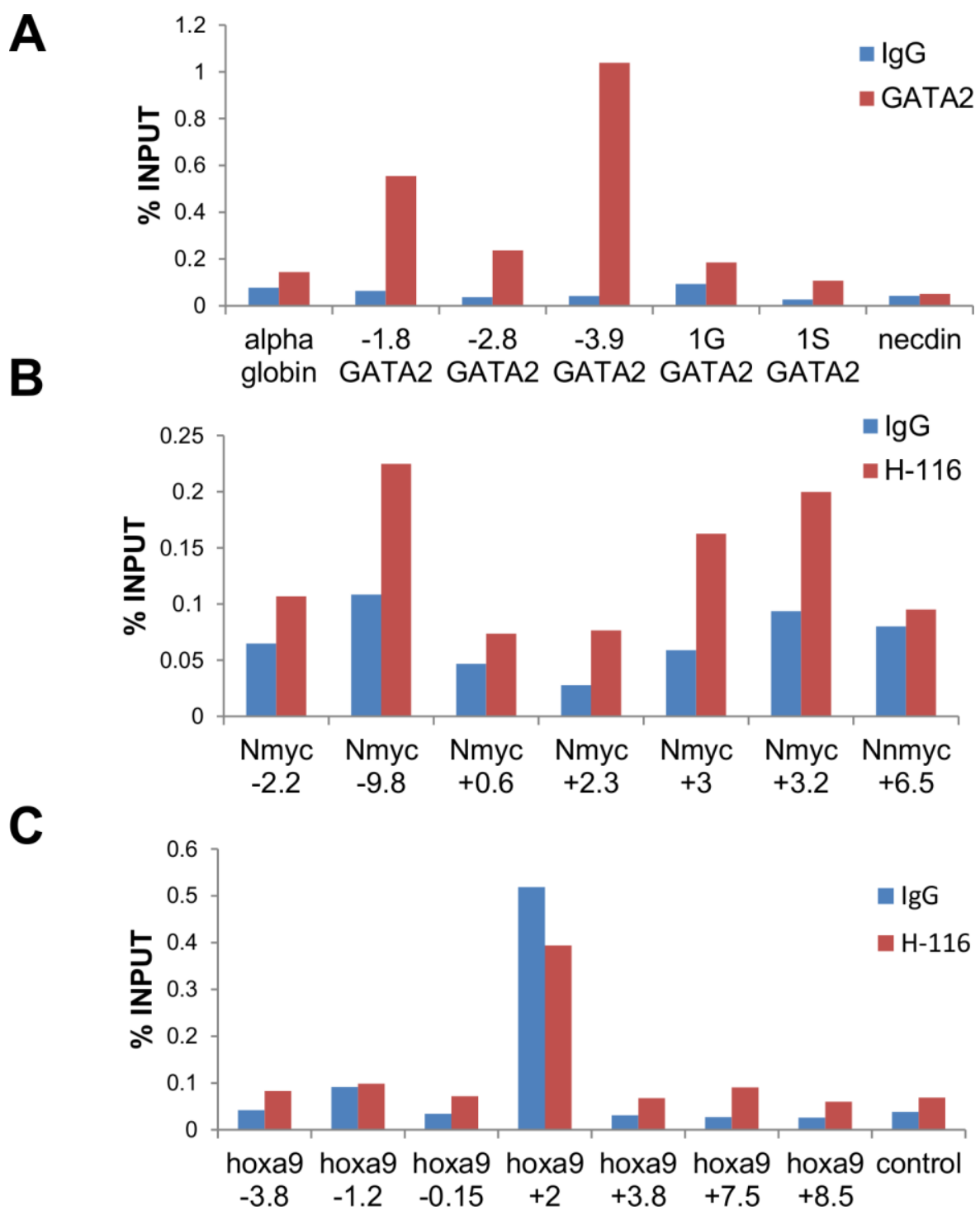

Figure 3-15. Absence of GATA2-ERT chromatin occupancy in the Nmyc and HoxA9 loci.

Quantitative Chromatin Immunoprecipitation (Chip) Analysis was performed to measure the GATA2-ERT occupancy in the GATA2 (A), Nmyc (B) and HoxA9 (C) loci in a GATA2-ERT cell line. The ChIP was performed using a rabbit polyclonal GATA2 antibody (H-116, Santa Cruz) and as a negative control ChIP was also performed with isotype matched preimmune IgG (IgG). The locations of the GATA2 binding sites tested are indicated relative to the Transcriptional start site of the gene. The $-1.8,-2.8$ and -3.9 GATA 2 binding sites are previously reported GATA2 binding sites and were used as positive controls for the assay. The $1 \mathrm{~S}$ and $1 \mathrm{G}$ alternate first exons of GATA2 and the brain specific promoter (necdin) are previously reported non-GATA2 binding sites. We also designed primers for a hoxA9 locus region which does not have WGATAR motif (control) as an additional negative control. 
predicted GATA2 binding sites containing the consensus "WGATAR" sequence. In the Nmyc locus we designed primers to test the regions which have 2 palindromic GATA2 sites together with E-Box motif (CANNTG) which is shown to have higher predictability of GATA2 binding. In the HoxA9 locus we tested all the 7 predicted GATA2 binding sites. Surprisingly there was no enrichment seen in any of the sites tested in the HoxA9 and Nmyc locus (Figure 3-15B and $\mathbf{C}$ ). These results indicate that HoxA9 and Nmyc are probably not direct targets of GATA2 but could be secondary or tertiary targets. It is also possible that GATA2 binds to distal regulatory elements such as enhancers to regulate Nmyc and HoxA9 expression.

\section{Lymphoid Block and Myeloid Expansion in Recipients of GATA2-ERT Transduced Cells}

The effects of the GATA2-ERT protein expression on murine hematopoiesis was studied by BM transplant studies. BM cells isolated from 5-FU treated mice were transduced with the GATA2-ERT and control GFP vector at similar MOI. The transduced cells were transplanted into lethally irradiated recipients in presence or absence of four fold excess of competing mock cells. At 12 weeks post-transplant GFP marking in the peripheral blood of recipients were analyzed by flow cytometry.

Recipients of undiluted control GFP vector transduced cells show similar GFP marking in B220+ cells $(58 \% \pm 20)$, Thy $1.2+$ cells $(58 \% \pm 15)$ and Gr1 $1+$ myeloid cells $(84 \% \pm 17)$

(Figure 3-16). In contrast the undiluted GATA2-ERT recipients show a very low GFP marking in B220+ cells $(8 \% \pm 3)$ and Thy $1.2+$ cells $(14 \% \pm 1)$ with high marking in Gr1+ cells $(91 \% \pm 3)$. This data indicate that the GATA2-ERT expressing HSCs preferentially differentiate into myeloid cells. The GFP mean fluorescence intensity in the B220+ cells and Thy1.2+ cells of GATA2-ERT recipients were much lower than the myeloid cells indicating that the cells which escape the lymphoid block have very low GATA2-ERT expression) (data not shown). The recipients of the diluted GFP vector transduced cells showed the expected lower percentages of GFP marking in all lineages compared to the undiluted group (B-cells $6 \% \pm 3$, T-cells $15 \% \pm 4$, myeloid $28 \% \pm 18$ ). However the diluted GATA2-ERT group still showed higher GFP marking in Gr1+ myeloid cells $(82 \% \pm 6)$ even after four fold dilution with mock cells. None of the transplant animals developed leukemia. These results demonstrate that GATA2-ERT provides a competitive advantage to myeloid cells in addition to a block in lymphoid differentiation.

\section{GATA2-ERT Blocks the Lymphoid Development after Common Lymphoid Progenitors (CLPs) and also Expands the Granulocyte Monocyte Progenitors (GMPs)}

Having found that the GATA2-ERT expands myeloid cells and blocks lymphoid differentiation the question remained as to the stage at which GATA2-ERT exerts its effects in both lineages. To investigate this we used the GATA2-ERT transgenic mice which were generated by lentiviral transduction of murine blastocysts. The GATA2-ERT transgene carried in the mice is driven by the same MSCV promoter as the retroviral 


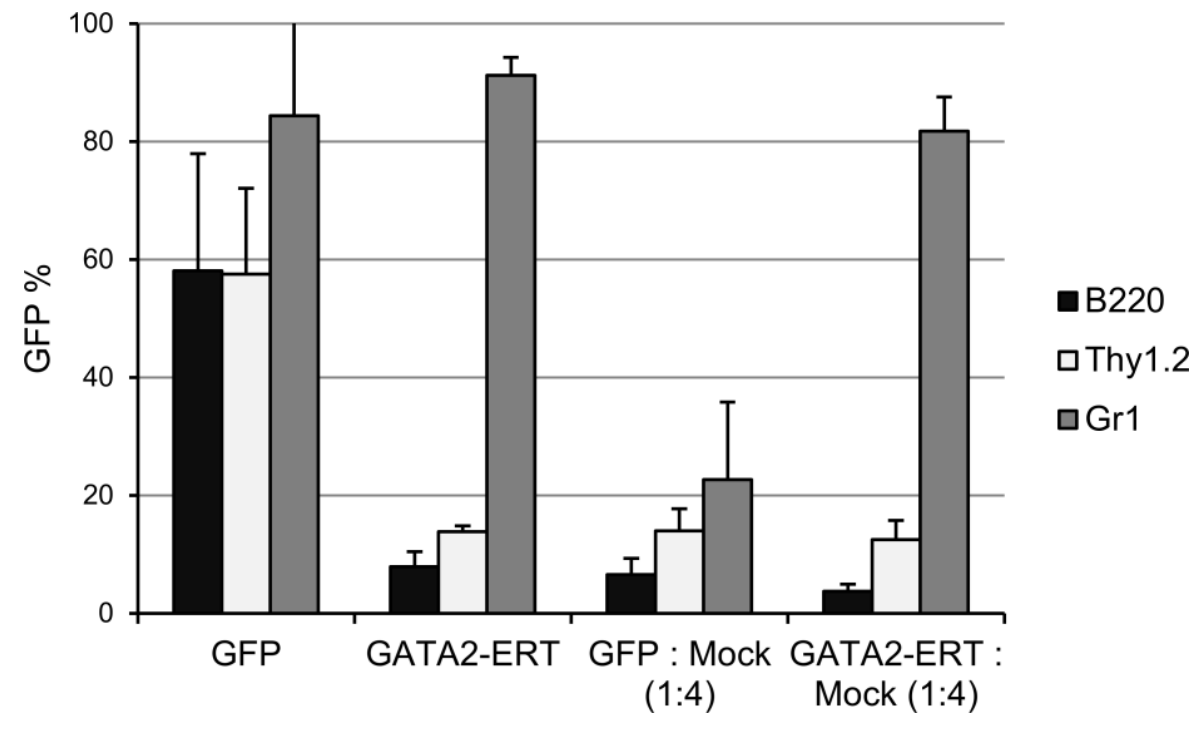

Figure 3-16. Lymphoid block and myeloid expansion in recipients of GATA2-ERT transduced BM.

FACS analysis of GFP percentages in B220 + cells, Thy $1.2+$ cells and Gr1 + cells in peripheral blood of recipients 12 weeks post-transplant. Values are represented as Mean \pm S.D . Total numbers of recipients in each group is as follows, GFP group $(n=2)$, GATA2-ERT group $(n=6)$, GFP 1:4 group $(n=7)$ and GATA2-ERT 1:4 group $(n=10)$. 
vector used previously in the transplant experiments. Southern blot analysis revealed the presence of 2 copies of the transgene (data not shown). Quite similar to the transplant results, these mice consistently showed high GFP\% in the Gr1+ and Mac1+ cells and very low GFP\% in the B220+ and CD3+ cells in their peripheral blood (Figure 3-17A). The stem and progenitor cell compartments from the GATA2-ERT transgenic mice bone marrow were analyzed for GFP expression. The Lin-Sca1+Kit+ (LSK) compartment had $35 \%$ GFP + cells which indicated that the GATA2-ERT transgene was frequently inactivated by position-effect variegation during HSC specification (Figure 3-17A). Presence of GFP marking in the CLP $(70 \% \pm 17)$ in bone marrow and its absence in mature $\mathrm{B} 220+, \mathrm{CD} 4+$ and CD8+ cells in peripheral blood suggested a post-CLP block. In the myeloid lineage there was a progressive increase in vector-expressing cells with differentiation. The GMPs showed higher GFP\% $(72 \% \pm 6)$ compared to the CMPs $(36 \% \pm 4)$, suggesting that GATA2-ERT has a proliferative effect on the GMPs. To identify the block in T-cell development we tested the GFP marking in thymocytes of GATA2-ERT mice at different stages of differentiation. GFP + thymocytes were only detected at the earliest DN1 stage $(19 \% \pm 5)$ which is lower than that of the CLPs in the bone marrow (Figure 3-17B). These data suggest that T-cell development is blocked at a pre-thymic stage and the few GATA2-ERT expressing cells which seed the thymus remain in the DN1 stage.

To understand the molecular mechanisms behind the GATA2-ERT mediated lymphoid block, global gene expression profiling of GATA2-ERT expressing and nonexpressing LSKs from transgenic mice was performed. Our analysis showed 157 probe sets were up-regulated and 190 probe sets were down-regulated in the GATA2-ERT expressing LSKs. Furthermore, Gene Set Enrichment Analysis showed down-regulation of B cell differentiation, B-cell receptor signaling, IL7 pathways and primary immunodeficiency related pathways (Figure 3-17C). Crucial regulators of lymphoid fate (Vpreb1, Ebf1, IL7R, Rag1, Dntt, Btla, Rag2, Lef1, and Tcf1) were significantly downregulated in the GATA2-ERT expressing LSKs (Table 3-4).

\section{DNA Binding Activity of GATA2-ERT Is Required for Myeloid Expansion and Lymphoid Block}

It was possible that the myeloid expansion and the lymphoid block could be attributed either to the low level transcriptional activity of the nuclear GATA2-ERT or to cytoplasmic GATA2-ERT titrating out transcription factors required for lymphoid fate choice (Ikaros, EBF, E2A and Mef2c) by protein-protein interactions. To clarify the contribution from these two different processes we developed DNA binding mutants of GATA2-ERT and tested them in functional assays to ascertain whether the phenotypic effects required DNA binding of GATA2-ERT. Two point mutations C352P and C370P were made in the cysteine residues of the 2 nd zinc finger of GATA2 which is critical for DNA binding activity (Figure 3-18A and B). These mutations were designed based on previous studies to disrupt the structural integrity of this zinc finger and thereby abolish DNA binding activity. ${ }^{74,135,136}$ The C352P and C370P mutant proteins expressed at similar levels to the wildtype GATA2-ERT protein in transduced bone marrow cells 
A

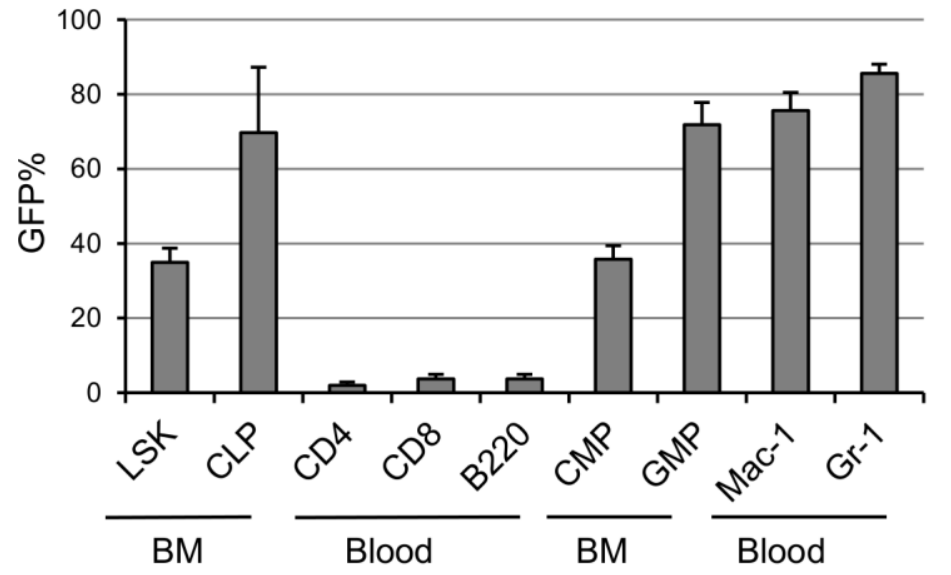

B

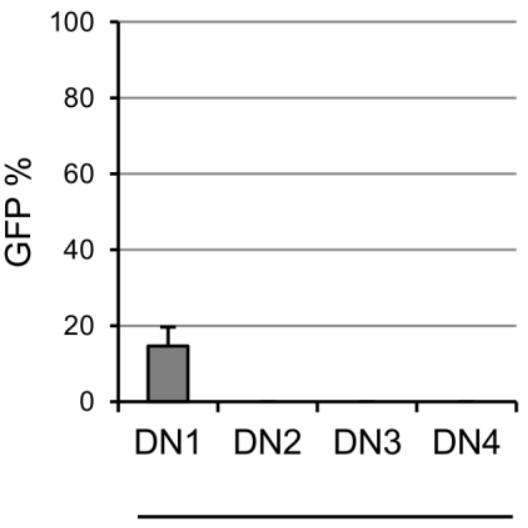

Thymus
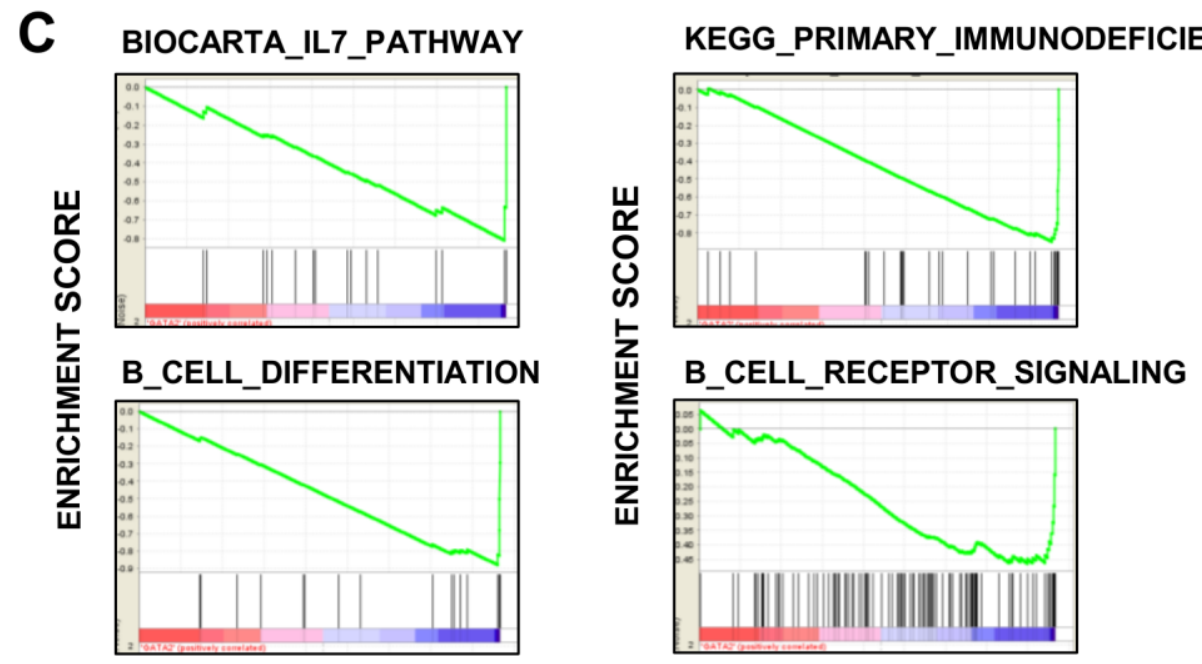

GATA2-ERT positively correlated

GATA2-ERT negatively correlated

Figure 3-17. GATA2-ERT blocks lymphoid development after CLPs and also expands GMPs.

(A) FACS analysis of GFP percentages in various lymphoid and myeloid progenitors and mature cells from GATA2-ERT transgenic mice $\mathrm{BM}$ and peripheral blood $(\mathrm{n}=3)$. $\mathrm{LSK}=$ Lineage-, Sca1+, c-Kit+; CLP = Lineage-, IL-7R ${ }^{+}$, Scal low, c-Kit low; CMP = Lineage-, Sca1-, c-Kit+, Fc $\gamma \mathrm{R}$ intermediate, CD34 intermediate; GMP = Lineage-, Sca1-, c-Kit+, Fc $\gamma$ R high, CD34 high. (B) FACS Analysis of GFP percentages in thymocytes from GATA2-ERT transgenic mice at various stages of differentiation $(n=6) . \mathrm{DN} 1=\mathrm{CD} 4$ -, CD8 -, CD44 +, CD25 -; DN2= CD4 -, CD8 -, CD44 +, CD25 +; DN3= CD4 -, CD8 -, CD44 -, CD25 +; DN4= CD4 -, CD8 -, CD44 -, CD25 -. (C) GSEA Analysis of gene expression profile between GFP - vs. GFP + LSK cells from BM of GATA2-ERT transgenic mice $(n=3)$. For each experiment RNA is isolated from sorted GFP + or GFP LSK cells derived from pooled BM (7 GATA2-ERT transgenic mice per experiment). Enrichment plots shown for selected down regulated gene sets identified by GSEA. 
Table 3-4. Changes in gene expression in GATA2-ERT expressing LSKs.

\begin{tabular}{|c|c|c|}
\hline Gene Symbol & Description & Fold Change \\
\hline Vpreb1 & pre-B lymphocyte gene 1 & -13.74 \\
\hline Igll1 & immunoglobulin lambda-like polypeptide 1 & -11.71 \\
\hline Ebfl & early B-cell factor 1 & -11.22 \\
\hline$B \operatorname{lnk}$ & B-cell linker & -8.47 \\
\hline$I l 7 r$ & interleukin 7 receptor & -7.65 \\
\hline Ragl & recombination activating gene 1 & -7.63 \\
\hline Vpreb3 & pre-B lymphocyte gene 3 & -5.62 \\
\hline Dntt & deoxynucleotidyltransferase, terminal & -5.18 \\
\hline Btla & $\mathrm{B}$ and T lymphocyte associated & -3.34 \\
\hline $\operatorname{Rag} 2$ & recombination activating gene 2 & -3.22 \\
\hline $\operatorname{Igh}-6$ & immunoglobulin heavy chain 6 & -3.09 \\
\hline$T c r g-V 4$ & T-cell receptor gamma, variable 4 & -2.74 \\
\hline Lef1 & lymphoid enhancer binding factor 1 & -2.71 \\
\hline $\operatorname{Igj}$ & immunoglobulin joining chain & -2.65 \\
\hline Tcrg-V2 / Tcrg-V3 & T-cell receptor gamma, variable 2 / variable 3 & -2.39 \\
\hline Tcf7 & transcription factor $7, \mathrm{~T}$-cell specific & -2.23 \\
\hline$H b b-b 1 / / / H b b-b 2$ & hemoglobin, beta adult major chain / minor chain & 3.33 \\
\hline $\operatorname{Igfl}$ & insulin-like growth factor 1 & 3.55 \\
\hline Hmoxl & heme oxygenase (decycling) 1 & 4.73 \\
\hline Earl / $12 / 2 / 3$ & $\begin{array}{l}\text { eosinophil-associated, ribonuclease A family, } \\
\text { member } 1 / 12 / 2 / 3\end{array}$ & 4.89 \\
\hline$A x l$ & AXL receptor tyrosine kinase & 5.00 \\
\hline Spic & Spi-C transcription factor (Spi-1/PU.1 related) & 5.21 \\
\hline Epx & eosinophil peroxidase & 5.34 \\
\hline$H b a-a 1 / / / H b a-a 2$ & hemoglobin alpha, adult chain 1 / chain 2 & 6.21 \\
\hline$C l q c$ & complement component 1 , q C chain & 10.63 \\
\hline Mrcl & mannose receptor, $\mathrm{C}$ type 1 & 16.06 \\
\hline$C l q b$ & complement component $1, \mathrm{q}$ beta polypeptide & 16.78 \\
\hline Clqa & complement component $1, \mathrm{q}$ alpha polypeptide & 20.52 \\
\hline Vcam1 & vascular cell adhesion molecule 1 & 28.75 \\
\hline
\end{tabular}

Individual Fold changes of genes differentially expressed in GFP + LSKs compared to GFP - LSKs (FDR $<0.05$ using LPE T-test) of GATA2-ERT transgenic mice. 


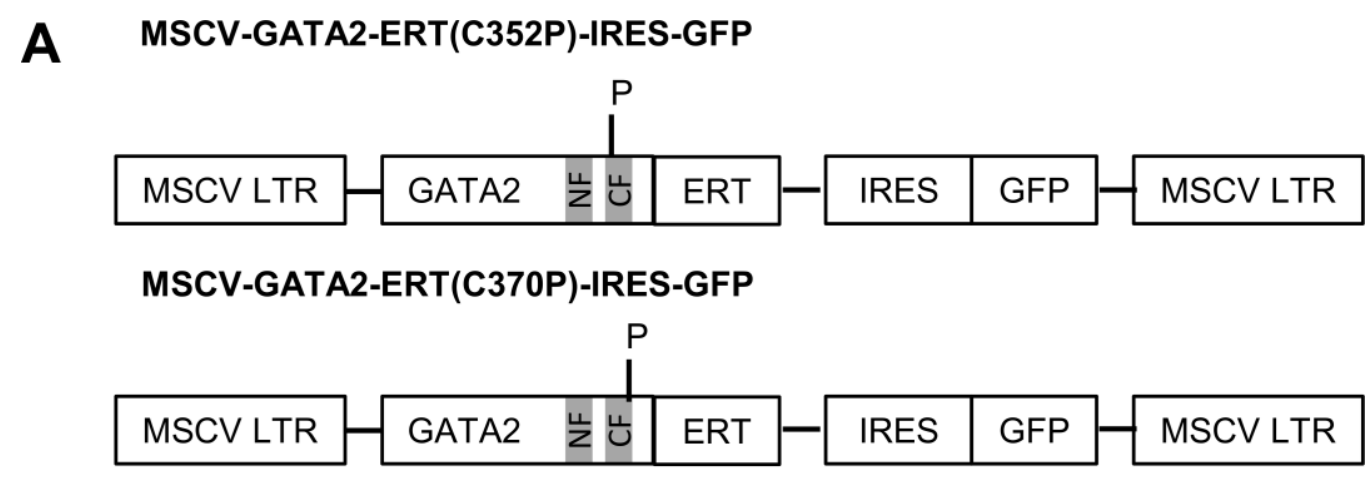

B

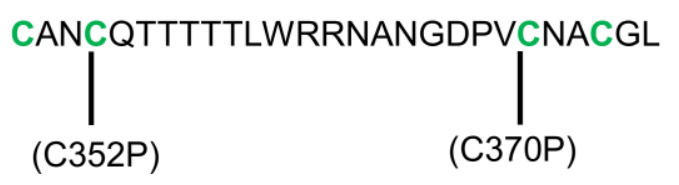

Figure 3-18. Schematic of the mutant GATA2-ERT retroviral vectors.

(A) Schematic of the GATA2-ERT retroviral vectors carrying the C352P and C370P point mutations. (B) Amino acid sequences of the C-terminal Zinc Finger with the locations of the point mutations. The four cysteine residues co-ordinating the zinc ion of the 2 nd finger are marked in green. 
(Figure 3-19A). Both the mutants also leaked into the nucleus of transduced BM cells similar to the wildtype GATA2-ERT (Figure 3-19B). However the C352P and C370P mutants showed a loss of DNA binding activity to the consensus WGATAR motif in gel shift assay compared to wildtype GATA2-ERT (Figure 3-20). We then tested the biological effects of the loss of DNA binding of the C352P and C370P mutations. Lack of DNA binding activity abolished the growth advantage of GATA2-ERT transduced BM cells over mock cells in liquid culture. GATA2-ERT wild type, two mutants and control GFP transduced BM cells were diluted with mock cells to achieve around $25 \% \mathrm{GFP}+$ cells. After 12 days in culture the percentage of GFP+ ve cells in the GATA2-ERT group was $91 \% \pm 6$ compared to $\mathrm{C} 352 \mathrm{P}$ and $\mathrm{C} 370 \mathrm{P}$ mutants and GFP group which remained at $32 \% \pm 7.5,40 \% \pm 10$ and $38 \% \pm 14$ (Figure 3-21A).

The $\mathrm{C} 352 \mathrm{P}$ and $\mathrm{C} 370 \mathrm{P}$ mutations also abolished the self-renewal capacity of GATA2-ERT in serial CFU-C assays. The cells harvested from the primary colonies of GATA2-ERT wild type group formed at least 7-fold higher secondary colonies than the 2 mutants and the control GFP groups (Figure 3-21B). Finally to test if DNA binding is required for myeloid expansion and lymphoid block in vivo, transplant experiments were performed as described above with the $\mathrm{C} 352 \mathrm{P}$ mutant. At 12 weeks post-transplant undiluted GATA2-ERT recipients showed low GFP marking in B220+ cells $(25 \%)$ and $\mathrm{CD} 3+$ cells $(50 \%)$ with high marking in $\mathrm{Gr} 1+\mathrm{Mac} 1+$ cells $(94 \%)$ when compared to the control undiluted GFP recipients showing uniform GFP marking in all three subsets (B220+ 76\%, CD3+ 73\%, Gr1+Mac1+ 84\%) (Figure 3-22A). The GATA2-ERT recipients also showed higher GFP MFI in the myeloid cells compared to the lymphoid cells indicating that there exists a dose dependent lymphoid block and only low expressing $\mathrm{B}$ and $\mathrm{T}$ cells escape the block (Figure 3-22B and $\mathbf{C}$ ). The undiluted $\mathrm{C} 352 \mathrm{P}$ mutant group show higher GFP marking in B220+ cells $(50 \%)$ and CD3+ cells $(46 \%)$ similar to the Gr1+ Mac1+ cells (75\%) resembling the GFP groups (Figure 3-22A). The C352P recipients also show similar GFP MFI between the myeloid and lymphoid subsets which show that the dose dependent lymphoid block requires DNA binding activity (Figure 3-22B and C). However since the GFP percentage and MFI of CD $3+$ ve cells is similar between the GATA2-ERT and C352P groups it is still possible that the T-cell block is mediated by protein-protein interactions. Upon four-fold dilution with mock cells only the GATA2-ERT wild type maintained high GFP\% in Gr1+ Mac1+ cells (62\%) compared to the C352P (19\%) and control GFP (34\%) groups. This demonstrates that the DNA binding activity of the GATA2-ERT is also required for myeloid expansion in vivo. 


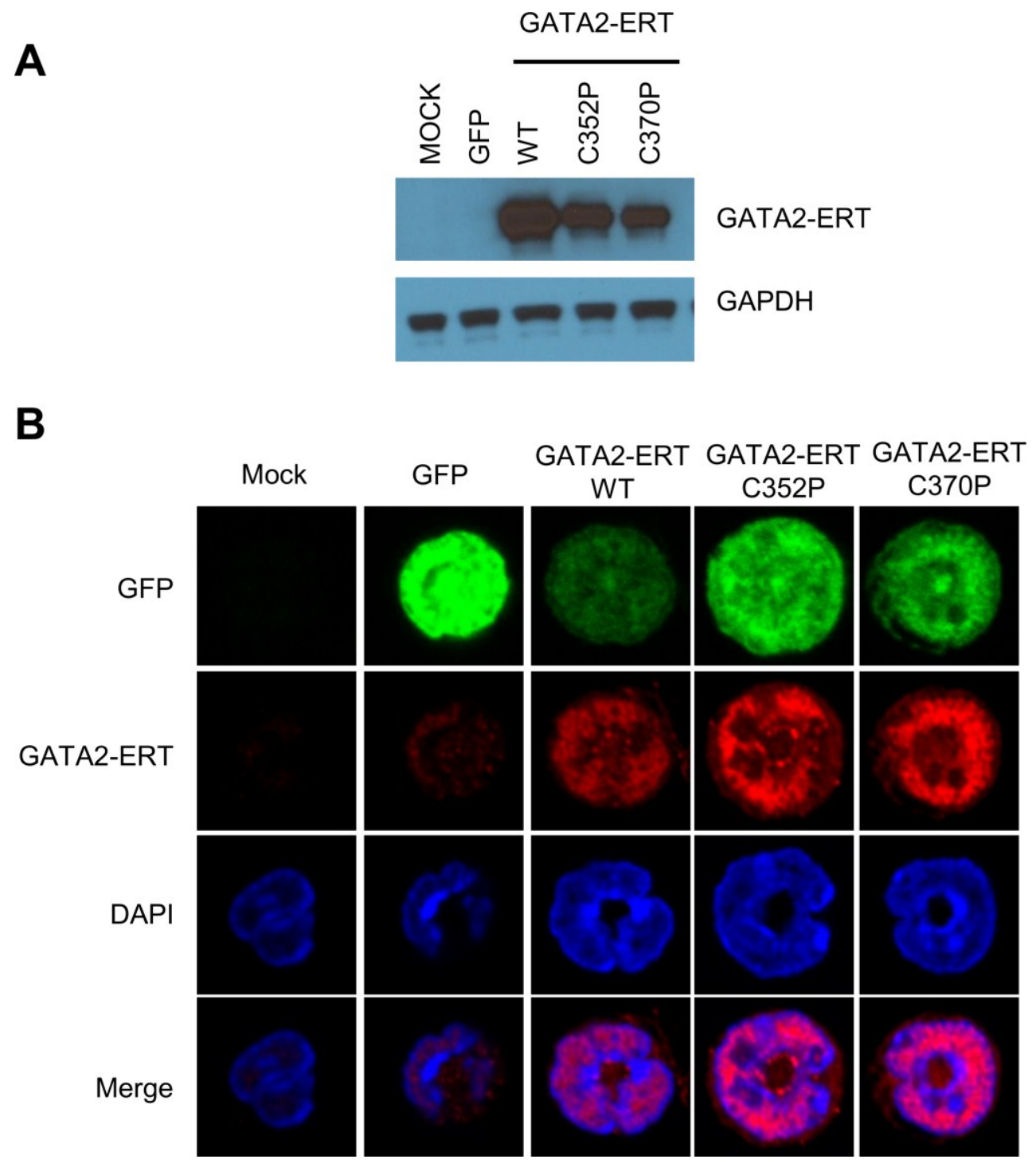

Figure 3-19. Expression and subcellular localization of GATA2-ERT mutants in primary BM cells.

Lin - BM cells were transduced with the wild-type and mutant GATA2-ERT vectors and control GFP vector at similar MOI. (A) At the 3 days post-transduction proteins were isolated and western blotted for GATA2-ERT ( $\alpha$ ER alpha) and GAPDH. All the groups had similar GFP percentages (60\% to $70 \%$ ). (B) Immunostaining was also performed at the same time point post-transduction. Cells were attached to retronectin coated coverslips for 40 minutes before fix and stain. Red represents GATA2-ERT $\left(1^{\circ}-\alpha\right.$ ER alpha, $2^{\circ}-\alpha$-rabbit Alexa Flour 555), blue represents DAPI and Green represents GFP. Imaging was performed using Zeiss LSM 510 Meta point scanning confocal microscope with a Plan Apochromat 63X/1.4 Oil objective and zoom scan of 3.0. 


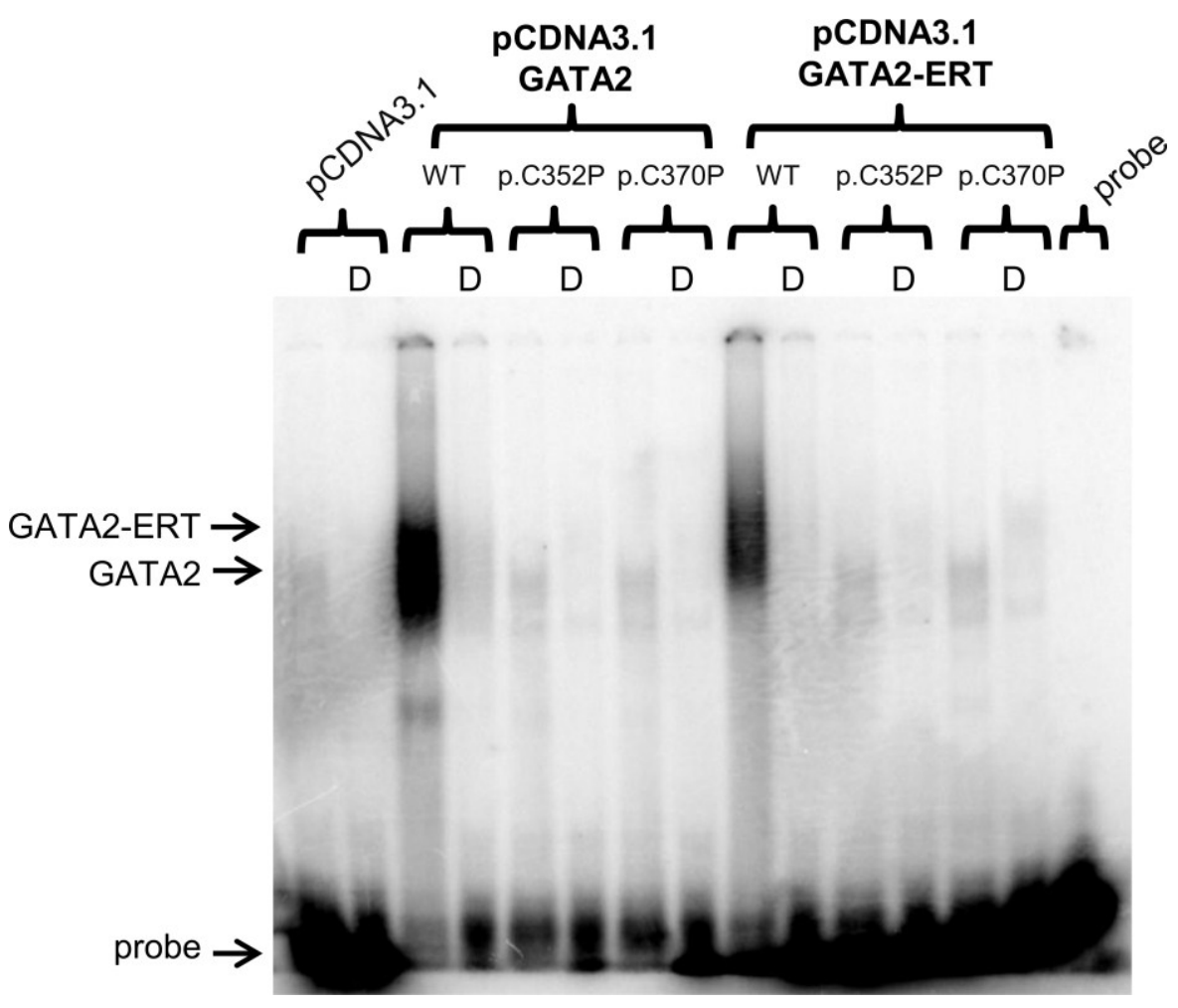

Figure 3-20. C352P and C370P are DNA binding defective mutants of GATA2. Electrophoretic Mobility Shift Assay (EMSA) of wild type and mutant GATA2 and GATA2-ERT proteins. GATA2 and GATA2-ERT proteins were synthesized from pCDNA3.1 expression vectors by in vitro transcription translation (TnT) and bound to $\mathrm{P}^{32}$ labeled mouse alpha globin promoter oligonucleotide (containing a GATA binding site) in the absence and presence of 500 fold unlabeled oligonucleotides (D). Position of free probe and protein DNA complexes containing GATA2 and GATA2-ERT proteins are indicated. 
A

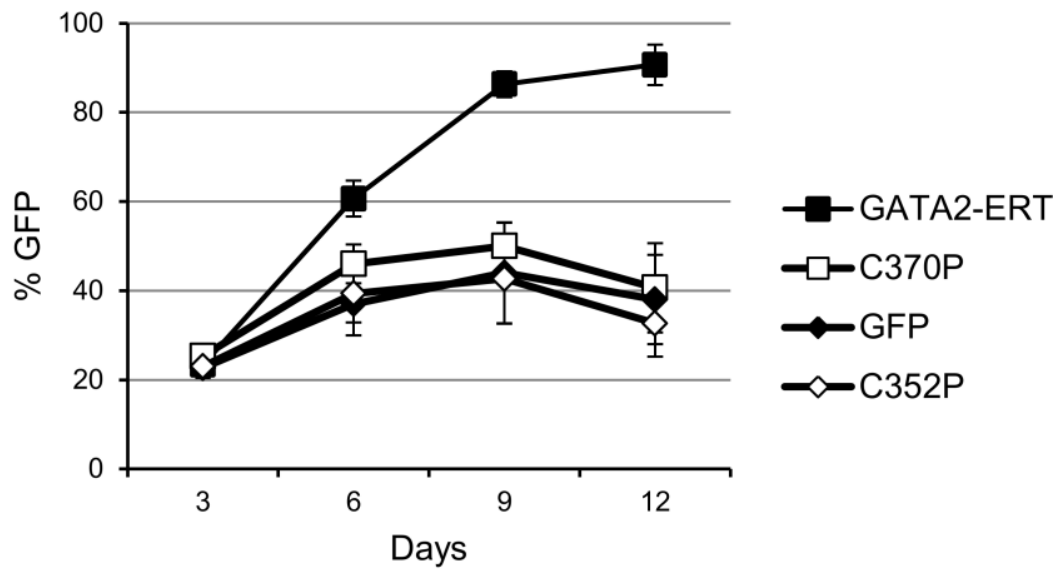

B

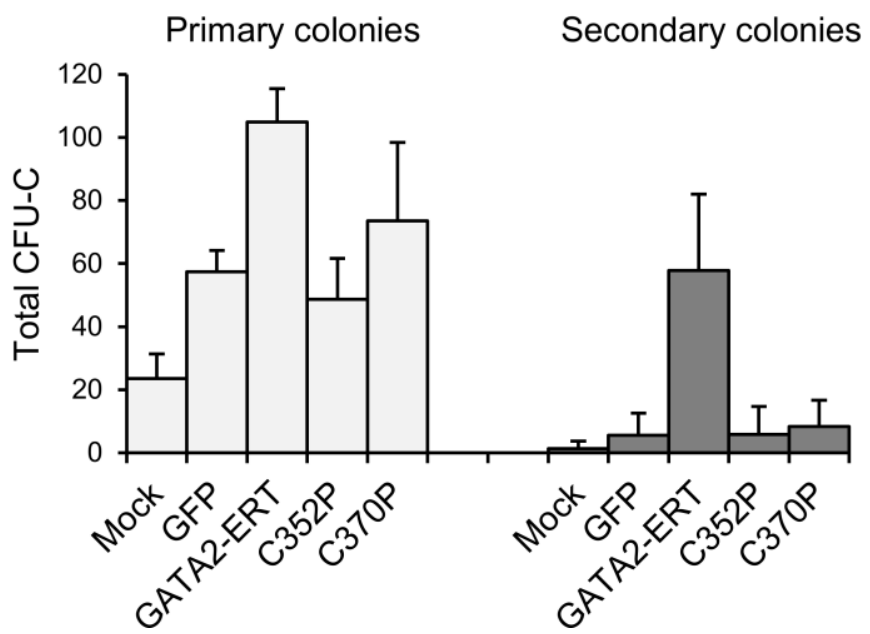

Figure 3-21. DNA binding activity of GATA2-ERT is required for myeloid expansion in vitro.

(A) FACS Analysis of GFP percentages of transduced lineage depleted BM cells at different time points in liquid culture after transduction $(n=3)$. Transduced cells were diluted at day 0 with mock transduced cells to start at a uniform GFP percentage $(25 \%$ to $28 \%$ ) in all samples. (B) Total CFU-Cs from primary and secondary colony plating of transduced lineage depleted BM cells. Cells were plated at 1.25 X103 per plate in the primary plating followed by $2.5 \times 104$ per plate for secondary plating. 

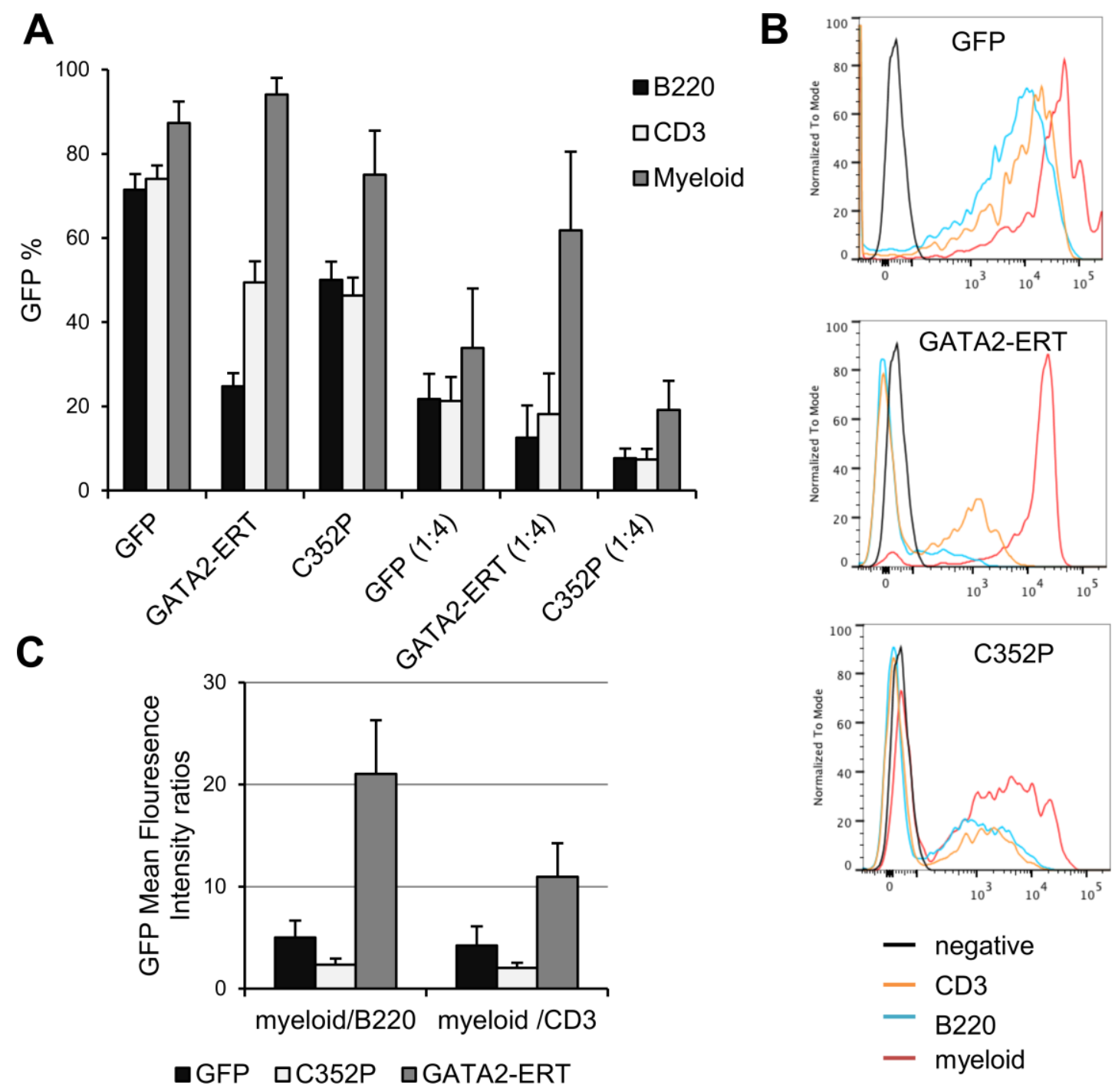

Figure 3-22. DNA binding activity of GATA2-ERT is required for myeloid expansion and lymphoid block in vivo.

(A) FACS analysis of GFP percentages in B220 + cells, CD3 + cells and Gr1 + Mac1 +ve cells (myeloid) in peripheral blood of recipients 12 weeks post-transplant. Values are represented as Mean \pm S.D of 10 recipients in each group. (B) Comparison of GFP expression between B220 + cells, CD3 + cells and Gr1+ Mac1+ cells (myeloid) in representative GFP, GATA2-ERT and C352P transplant recipients at 16 weeks posttransplant. (C) GFP Mean Fluorescence Intensity (MFI) Ratios in myeloid cells against $\mathrm{B} 220+$ and CD3 + cells in GFP, GATA2-ERT and C352P transplant recipients at 16 weeks post-transplant $(\mathrm{n}=10)$. 


\section{CHAPTER 4. DISCUSSION}

\section{GATA2 Dose and Lineage Commitment}

In this study, using retroviral transduction of GATA2-ERT vector into BM stem and progenitor cells followed by transplantation, we show that nuclear concentrations of the transcription factor GATA2 differentially regulate the development of granulocytes, T-lymphocytes and B-lymphocytes. High nuclear concentrations of GATA2 resulted in granulocyte development whereas moderate and low concentrations resulted in $\mathrm{T}$ lymphocyte and B-lymphocyte differentiation respectively. This conclusion was made from the observation that in the peripheral blood of GATA2-ERT transplant recipients, highest GFP expression (as measured by the MFI) was seen in the Gr1+ Mac1+ granulocytes, followed by a moderate GFP expression in the CD3+ T-lymphocytes and lowest GFP expression in the B220+ B lymphocytes.

Since the retroviral vector is bi-cistronic in which GFP is linked to GATA2-ERT by the ires, GFP and GATA2-ERT are co-expressed at the mRNA level. In the myeloid GATA2-ERT cell lines, a correlation at the protein level was also confirmed. In the knockdown experiments on the GATA2-ERT cell lines, high GFP expression in the "Non-silencing group "was consistent with the high GATA2-ERT protein levels compared to the "shGFP" group in which low GFP expression was consistent with low GATA2-ERT protein levels.

Our findings have two possible explanations. In the first scenario, in a myelolymphoid progenitor different doses of GATA2 results in different cell fates (Figure 4-1A). Alternatively, the lymphoid and myeloid differentiation can be initiated at the same dose of GATA2 but in order to complete the differentiation GATA2 needs to be downregulated to different levels in the myeloid and lymphoid lineages (Figure 4-1B). I favor the second explanation for the following reasons. Firstly, in the GATA2-ERT transgenic mice there was no difference in the GFP expression between the CLP and CMP and the lymphoid block occurs only after the CLP and ETP. The high GFP expressing myeloid cells were also selected only after the GMP stage (data not shown). Secondly, the endogenous GATA2 expression is very high in the multipotent progenitors (LSK cells) and seems to decrease upon differentiation to both the CLP and CMP. Even though endogenous GATA2 expression in the CLP is lower than the CMP; this low expression is not maintained but is even further reduced upon differentiation to the mature cells.

The hypothesis that GATA2 needs to be down-regulated to different levels for multi-lineage differentiation also agrees with the previous report from our lab that enforced expression of GATA2 at the "HSC dose" leads to a complete block in multilineage differentiation. In the previous study MSCV-GATA2iresGFP vector resulted in GATA2 expression similar to that of the Lin- Sca1+ HSCs. ${ }^{95}$ Endogenous GATA2 expression is highest in the HSCs and since the vector maintained the HSC dose, these HSCs did not differentiate. The current MSCVGATA2-ERTires GFP vector achieves the 

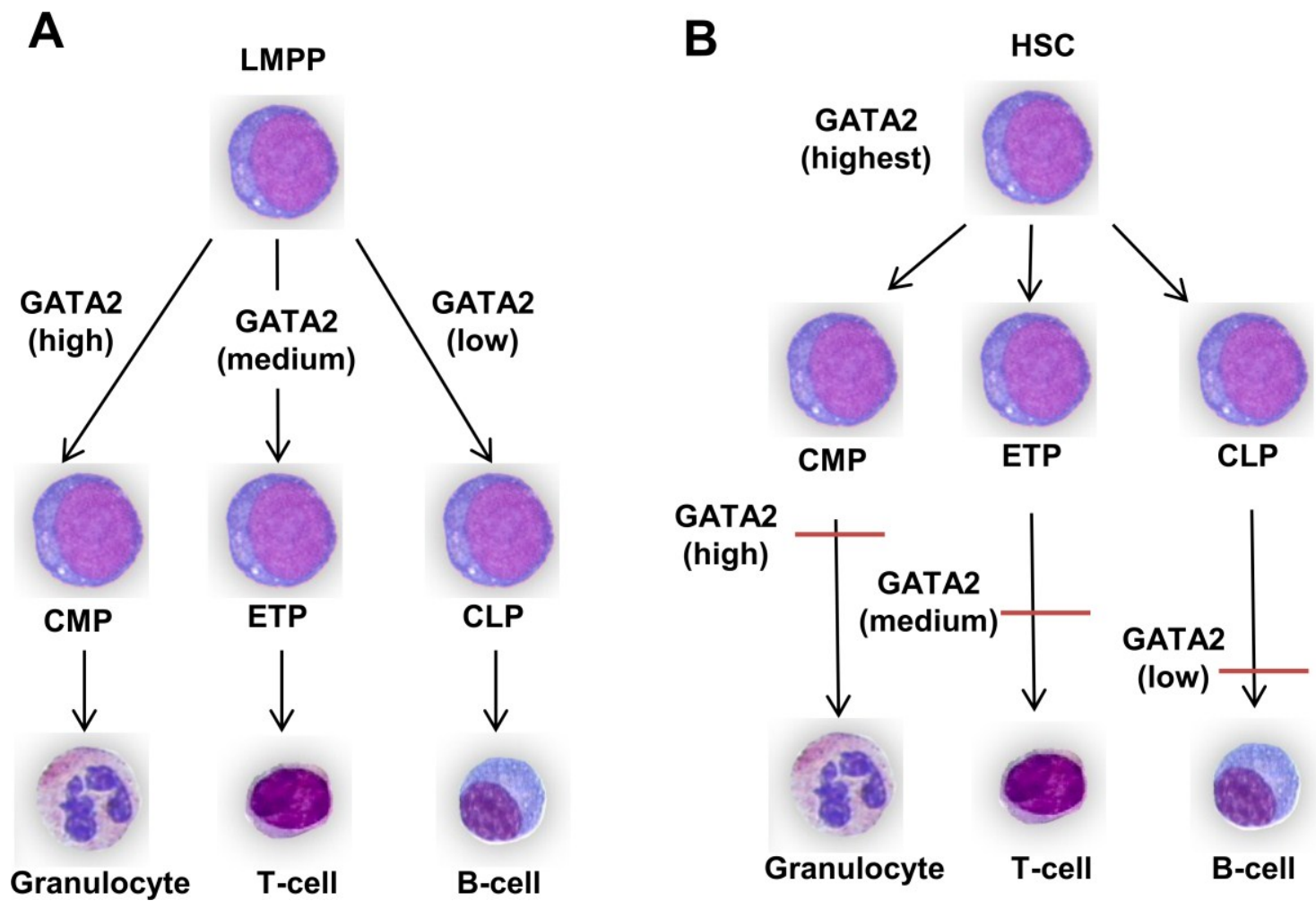

Figure 4-1. Models of GATA2 dose dependent lineage commitment.

(A) Different doses of GATA2 results in different cell fates in a myelo-lymphoid progenitor (LMPP). (B) Both lymphoid and myeloid differentiation can be initiated at the same dose of GATA2 but in order to complete the differentiation GATA2 need to be downregulated to different levels in the myeloid and lymphoid lineages. CMP: Common Myeloid Progenitor, ETP: Early Thymic Progenitor, CLP: Common Lymphoid Progenitor, HSC: Hematopoietic Stem Cell. 
same transgene mRNA expression, but achieves a much lower GATA2 nuclear protein levels due to the protein distribution between the nucleus and the cytoplasm. This resulted in normal multi-lineage differentiation and led to the identification of GATA2 dose effects further downstream in different lineages.

In the erythroid lineage GATA2 down regulation is mediated by GATA1. GATA1 is highly expressed in erythro-megakaryocytic lineages, whereas it is suppressed in HSCs and progenitor cell fractions. ${ }^{130}$ During erythroid differentiation GATA1 binds to regulatory elements in the GATA2 locus by displacing GATA2. This phenomenon called "GATA switch" results in down regulation of GATA2. ${ }^{87}$ The factors involved in GATA2 down regulation during myeloid and lymphoid differentiation still remain to be identified.

Dose dependent activity of transcription factors has been previously reported in normal hematopoiesis and leukemia. ${ }^{137}$ Best studied is the transcription factor PU.1. When Pu. $1^{-/}$fetal liver hematopoietic progenitors were transduced with retroviral vector carrying PU.1 -GFP, the rescued Pro B-cells and macrophages had high and low GFP expression respectively. ${ }^{46}$ The authors showed that low PU.1 protein is associated with a B lymphoid fate and a high PU.1 protein was associated with a macrophage fate.

Gradual decrease in GATA2 expression show that the HSC transcription program does not terminate abruptly during differentiation but persists during many stages. Myeloid lineage may be more permissive to the HSC program than the lymphoid lineage. This can explain the lower GATA2 threshold in the lymphoid lineage compared to the myeloid lineage.

\section{GATA2 and HSC Quiescence}

The HSCs remain highly quiescent compared to the progenitor cells which have prodigious proliferative capacity. Detailed cell cycle analysis and BrDU labeling assays revealed that most of the HSCs are non-dividing with only $1-3 \%$ in cycle and approximately $90 \%$ in G0 phase. ${ }^{11,138,139}$ A quiescent HSC population that divides only 5 times during the lifespan of the mice was also demonstrated. ${ }^{140}$ Quiescence has been postulated to prevent stem cell exhaustion and is thought to protect stem cells from acquiring mutations leading to their malignant transformation to putative cancer stem cells.

Several components of the cell cycle machinery like cyclin dependent kinase inhibitors (p21, p27, p16 and p18), D type cyclins and their associated kinases (Cdk4 and Cdk6) have been shown to be critical for HSC quiescence. ${ }^{141}$ However the transcriptional control of these components of cell cycle machinery still remains to be elucidated. Our data show that GATA2 inhibits cell cycle in BM cells in a dose-dependent manner. In the GATA2-ERT cell lines we observe a higher nuclear concentration in the presence of TAM and a lower nuclear concentration in the absence of TAM. This high GATA2 concentration resulted in a cell cycle arrest at G0/G1 phase. Similarly in CFU-C assays, 
in the presence of TAM there is no colony formation and in the absence of TAM there is an increased self-renewal of CFU-C forming progenitors

Our data is consistent with the previous studies with GATA2-ER vectors. When GATA2-ER was expressed in hematopoietic cell lines (FDCP Mix, BaF3 and 32D) and primary murine BM cells and Sca+ Lin- progenitors it resulted in a ligand induced block in proliferation and cell cycle arrest. ${ }^{106,142}$ The GATA2-ERT mediated quiescence in BaF3 cells was shown to be reversible upon removal of Tamoxifen. ${ }^{96}$ High expression of GATA2 is associated with quiescence in both human and mouse HSCs. Two fold higher GATA2 expression was found in quiescent compared to cycling CD34+ CD38- human cord blood HSCs. ${ }^{96}$ Higher GATA2 expression was found in the quiescent adult mouse BM HSCs compared to the cycling fetal liver HSCs. ${ }^{143}$ GATA2 expression was progressively down-regulated when the adult BM HSCs were induced to cycle after 5-FU treatment and returned to normal when the quiescence was reset. ${ }^{143}$ All these data strongly suggest that GATA2 could mediate HSC quiescence in a dose dependent manner.

\section{GATA2 Mediated Myeloid Expansion}

Persistent GATA2 expression not only favors a granulocyte cell fate choice but it also expands myeloid cells. GATA2-ERT expressing BM cells effectively out-compete mock transduced cells under myeloid conditions in vitro. In competitive transplant experiments, GATA2-ERT expressing cells also effectively out-compete mock transduced cells in the myeloid compartment. Even after transplanting GATA2-ERT expressing BM cells at 1:4 dilutions with mock cells, the recipients had an $82 \%$ GFP marked Gr1+ Mac1+ granulocytes.

The myeloid expansion is due to GATA2-ERT mediated increased self-renewal of the myeloid progenitors. In myeloid CFU-C assays, GATA2-ERT expressing myeloid progenitors formed larger size colonies which can further form colonies upon serial replating. This increased ability to form secondary colonies is a sign of increased selfrenewal. The myeloid progenitor that acquired increased self-renewal is probably the Granulocyte Monocyte Progenitor (GMP).This agrees with the observation that myeloid expansion in the GATA2-ERT transgenic mice starts at the GMP stage. This is also consistent with the previous reports that GATA2 ${ }^{+/} \mathrm{BM}$ have reduced cellularity in the GMP compartment and formed reduced GM colonies in CFU-C assays. ${ }^{144}$

It is also possible that the increased self-renewal of myeloid progenitors by retroviral transduction of MSCV GATA2-ERTires GFP vector can be due to "insertional mutagenesis". Insertion of a gamma retrovirus neighboring an oncogene can be followed by its aberrant expression due to the enhancer effects of the viral LTR elements. Alternatively the insertion of the gamma retrovirus can also disrupt and inactivate a tumor suppressor gene. Both these effects can cause myeloid expansion independent of GATA2-ERT. However loss of GATA2-ERT in the GATA2-ERT transduced cell lines leads to loss of the self-renewing Gr1 negative progenitor population. This is followed by 
complete differentiation into mature Gr1 positive cells and loss of proliferation in these cell lines. These data suggests that GATA2-ERT expression is essential for the continued proliferation and self-renewal of the myeloid progenitors and also that GATA2-ERT plays a direct role in these effects. It still remains possible that GATA2-ERT could exert these effects in co-operation with an un-identified gene which was insertionaly mutagenized. However GATA2-ERT vector immortalizes BM cells at a very high frequency than the GFP vector in vitro and also expands myeloid cells consistently in all transplant recipients. It is unlikely that the GATA2-ERT vector integrates next to the same co-operating gene in multiple experiments further arguing that these effects are mediated by GATA2-ERT alone.

Requirement of high expression of Nmyc and HoxA9 in the GATA2-ERT cell lines suggest that Nmyc and hoxA9 could be potential GATA2 targets in myeloid progenitors. Enforced expression of Nmyc by itself increased the proliferation and selfrenewal of myeloid progenitors. ${ }^{145}$ These effects were thought to be mediated by downregulation of TGF- $\beta$ signaling, upregulation of Smad7 and JNK signaling pathways. ${ }^{145}$ However these pathways were not affected by GATA2-ERT mediated Nmyc overexpression suggesting that the mechanism of Nmyc action could be different in the context of GATA2. Overexpression of HoxA9 is common in human mixed lineage leukemia (MLL) and is required for MLL fusion protein mediated transformation in a murine bone marrow transduction/transplantation model. ${ }^{146,147}$ Overexpression of HoxA9 in the BM cells expands myeloid cells with a block in lymphoid development at the PreB cell stage. ${ }^{148}$ Overexpression of HoxA9 also immortalized BM cells into GM-CSF dependent pro-myelocyte cell lines. ${ }^{149}$ Since the GATA2-ERT induced myeloid expansion is dependent on its DNA binding activity, GATA2-ERT could possibly bind to both Nmyc and HoxA9 locus resulting in their up-regulation. However the absence of GATA2-ERT chromatin occupancy in both the Nmyc and HoxA9 locus suggests that both Nmyc and HoxA9 could both be secondary targets of GATA2. Alternatively GATA2 could still bind to distant regulatory elements in the Nmyc and HoxA9 locus which were not tested in the ChIP assay. Such distal binding sites could be identified by performing ChIP-Seq analysis.

\section{GATA2 Mediated Lymphoid Block}

Gene expression analysis on various hematopoietic stem and progenitor populations has shown a low promiscuous expression of genes from different lineages that the multipotent cell can potentially differentiate into. ${ }^{150-152}$ This process is called "Lineage Priming". For example both lymphoid and myeloid specific genes were expressed in a Lympho-myeloid progenitor (LMPP) and both T-cell and B-cell lineage genes were expressed in a CLP. This is thought to be a consequence of alterations in chromatin structure which allows accessibility to various transcriptional regulators. This could also be due to the simultaneous expression of TFs of the respective conflicting lineages being expressed at low doses. 
Loss of lymphoid lineage priming in the MPPs has been associated with a block in lymphoid differentiation in a few gene knockouts. Ikaros knockout mice show an absence in B-cell development and T-cell defects. Ikaros had been shown to be essential for priming of lymphoid genes throughout the lymphoid differentiation starting from the HSC. ${ }^{153}$ One mechanism is thought to be chromatin remodeling because Ikaros interacts with the Nucleosome remodeling complex (NuRD) ${ }^{154}$ Recently it has been shown that Ikaros needs to interact with the GATA family of transcription factors and Cdk9 protein for appropriate chromatin binding and activation of its target genes. ${ }^{155}$ Ikaros was shown to interact with GATA2 in hematopoietic progenitors, GATA1 in erythroid cells and GATA3 in lymphoid cells. One mechanism by the sustained GATA2 expression in the MPPs and/or CLP can prevent Ikaros GATA3 binding thereby preventing Ikaros mediated lymphoid priming. Other gene knockouts which can cause lymphoid priming defects in the MPPs are Mef2c and E2A. ${ }^{156,157}$ Both Mef2ca and E2A are required for lymphoid differentiation.

Gene expression analysis on GATA2-ERT expressing MPPs show a downregulation of lymphoid specific signaling (IL7R), transcription factors (Ebf1,Lef1,Tcf7) and effectors (Rag1,Rag2,Dntt,Btla,Vpreb1 ,Igh-6,Tcrg ). There was a considerable overlap of genes downregulated in the Mef2 $\mathrm{c}^{-/}$MPPs and GATA2-ERT expressing MPPs and that were E2A or EBF targets (Rag1, Dntt, Igh-6 and Tcf7). ${ }^{157} \mathrm{We}$ hypothesize that sustained expression of GATA2 could antagonize the activity of transcription factors E2A, Mef2c and EBF to prevent lymphoid priming in the MPPs. Since the block in B-cell differentiation mediated by GATA2-ERT is dependent on its DNA binding activity, it is possible that GATA2 could bind to these lymphoid specific genes loci and prevent their transcription. GATA2 could additionally prevent the binding of other lymphoid specific TFs at these loci.

It is not clear whether the T-cell block is due to DNA binding activity of GATA2.The C352P transplant recipients showed similar GFP marking in T-cells compared to the wild type GATA2-ERT recipients. However the T-cell GFP marking approximates the myeloid GFP marking in the C352P recipients than the wildtype GATA2-ERT recipients. It is possible that titration of a T-cell lineage specific TF by GATA2-ERT in the cytoplasm due to protein-protein interaction can lead to the Tlymphoid block. The lymphoid priming of such TFs will possibly be unaffected by GATA2-ERT. Some of the lymphoid specific transcription factors like Ikaros, GATA3 and Ets1 were not differentially regulated in the MPP and could be potential candidates in this context.

\section{GATA2 Overexpression in Acute Myeloid Leukemia (AML)}

GATA2 overexpression is frequently observed in Acute Myeloid Leukemia. In a recent study with a large series of AML patients GATA2 was overexpressed in $37.4 \%$ of cases (97/259) as measured by real-time RT-PCR. ${ }^{158}$ GATA2 overexpression was also significantly associated with FLT3-ITD and NPM1 mutations and overexpression of WT1 and Evi1. ${ }^{158}$ In another study with pediatric AML patients GATA2 was 
overexpressed in $65 \%$ of cases $(155 / 237) .{ }^{159}$ During complete remission GATA2 expression returned to normal but remained high in patients with resistant disease. ${ }^{159}$ GATA2 overexpression also correlated with a poor prognosis in patients receiving chemotherapy in both studies. GATA2 overexpression could possibly transform a normal myeloid progenitor cell like CMP or GMP into a Leukemic Stem cell (LSC) by conferring high proliferation and self-renewal capacity and also leading to additional mutations. Alternatively GATA2 overexpression can be a part of gene expression program inherited from a HSC which was transformed into a LSC by a different driver mutation.

Expression of many leukemic gene fusions like MLL-ENL, MLL-AF9, MOZTIF2 and NUP98-HOXA9 can cause increased self-renewal of myeloid progenitors, immortalization of primary BM cells in vitro, and leukemia formation upon transplantation. ${ }^{160-164}$ In our study expression of GATA2-ERT immortalized primary BM cells into IL-3 dependent myeloid cell lines. GATA2-ERT expression also increased the self-renewal and proliferation of myeloid progenitors. However no leukemia was observed upon transplantation. The GATA2-ERT transgenic mice even when crossed with $\mathrm{p} 16 / \mathrm{p} 18 \mathrm{dko}$ mice or $\mathrm{p} 21 / \mathrm{p} 27 \mathrm{dko}$ did not show an increase in incidence of leukemia generation. These data suggest that additional cooperating mutations that can block differentiation may be required to transform GATA2-ERT expressing myeloid progenitors into LSCs.

Targeting the GATA2 mediated transcriptional network in the LSCs could be a potential strategy in AML treatment. In our studies knockdown of GATA2-ERT and its targets hoxA9 and Nmyc, in the immortalized cell lines differentiated these cells and stopped their proliferation. It will be interesting to test if patient derived AML cell lines are dependent on GATA2 for their proliferation. Such a strategy was employed in Ras oncogene driven Non-small cell lung cancers (NSCLC) which required GATA2 for their survival. ${ }^{165}$ GATA2 was found to transcriptionally regulate $\mathrm{NF \kappa B}$, proteasome and Rho signaling pathways and targeting these pathways reduced tumor development in lung. ${ }^{165}$ In our gene expression studies on GATA2-ERT cell lines we did not identify these pathways suggesting that mechanisms could be different in hematopoietic neoplasms.

\section{GATA2 Mutations in Familial AML/MDS Syndromes}

Mutations in GATA2 have been identified recently in rare familial disorders like DCML, MonoMAC syndrome and Emberger syndrome all of which have a predisposition to MDS and AML. ${ }^{19-121}$ DCML and MonoMAC syndromes are closely related syndromes characterized by monocytopenias, B-cell and NK cell lymphopenias associated with mycobacterial, fungal and viral infections and predisposition to MDS/AML ${ }^{166,167}$ Emberger syndrome is characterized with severe lymphedema in addition to predisposition to MDS and AML.GATA2 mutations were also associated with familial MDS/AML syndromes without any other hematopoietic and vascular defects. ${ }^{118}$ All these syndromes show an autosomal dominant inheritance with incomplete penetrance. 
Mutations of GATA2 found in these syndromes are highly heterogeneous ranging from deletions, frame-shift mutations leading to premature termination and point mutations. Since one wild-type allele was always present, some of the mutations (deletions and frame-shift) were thought to result in GATA2 haplo-insufficiency and lead to the disease. However in the case of point mutations the question remained whether the mutation could be dominant negative rather than a loss of function. Almost all of the point mutations were found in the $2^{\text {nd }}$ zinc finger domain which is important for DNA binding of GATA2 and protein-protein interactions. The Thr354Met and Thr355Del mutations both showed loss of DNA binding activity in gel shift and luciferase assays. ${ }^{118}$ Surprisingly Thr354Met showed some dominant negative effects in ATRA induced differentiation of HL-60 cells. ${ }^{118}$

In our study we artificially designed two GATA2 DNA binding mutants (C352P and $\mathrm{C} 370 \mathrm{P}$ ) with mutations in the $2^{\text {nd }}$ zinc finger. Both these mutants abolished the myeloid expansion and lymphoid block mediated by sustained GATA2 expression. These results favor the hypothesis that the $2^{\text {nd }}$ zinc finger missense mutations found in patients are probably loss of function mutations and the disease is caused by GATA2 haploinsufficiency. GATA $2^{+/-}$mice are viable but have "smaller HSC pool size" due to defects in HSC specification. ${ }^{91}$ Under stress conditions like infections, due to enormous requirement of mature cells, there can be a HSC exhaustion leading to MDS and finally AML.

In our study the sustained GATA2 expression leads to B-cell specific block. This block is not associated with monocytopenia but rather an expansion of $\mathrm{Gr}-1+$, mac1+ cells. In contrast in the DCML and MonoMAC syndromes the B-cell deficiency is associated with deficiency of CD14+ monocytes and NK cells. This suggest that the mechanism of B-cell block in DCML and MonoMAC is probably not sustained GATA2 expression in CLP but a GATA2 haplo-insufficient HSC under stress generating less mature cells. BM analysis of MonoMAC patients also showed absence of multi-lymphoid progenitors and GMPs confirming this hypothesis. ${ }^{166}$

\section{Technical Considerations in Using the GATA2-ERT System}

In the absence of TAM, GATA2-ERT protein is localized in both the nuclear and cytoplasmic fractions of BM cells. The leaky nature of the GATA2-ERT protein calls for two explanations for the observed phenotype. DNA binding and transcriptional regulation by the GATA2-ERT leaking into the nucleus or the retention of other factors by the cytoplasmic GATA2-ERT. To distinguish between these two mechanisms we used the DNA binding GATA2-ERT mutants, C352P and C370P. These two mutations were made in the cysteine residues of 2 nd zinc finger domain of GATA2. The C352P was used previously by other groups to study functions of both GATA1 and GATA2. ${ }^{74,135,136}$ The myeloid expansion in vitro (CFU-C replating assay and proliferation assay) and in vivo (competitive transplants) were completely dependent on DNA binding of the leaky GATA2-ERT. The B lymphoid block was also dependent on DNA binding activity of 
GATA2-ERT. On the other hand it is unclear whether T-cell block is due to DNA binding activity or protein-protein interactions.

The GATA2-ERT system we used contains a human GATA2 cDNA that was overexpressed in mouse hematopoietic tissues. Both human and mouse GATA2 are 480 amino acids long with $98 \%$ similarity in amino acid composition. None of the amino acid changes are located it the critical zinc finger domains suggesting that both the mouse and human GATA2 probably behaves similarly in functional assays. In the GATA2-ERT transgenic mice, the developing immune system should recognize the human GATA2 protein as self-protein and do not develop antibodies against it. Similarly the transplant recipients should also tolerate human GATA2 expressing BM cells since they received immune ablation before transplant.

Even though the GATA2-ERT system is advantageous because it achieved low doses of GATA2, the system has some drawbacks. It is possible that the GATA2-ERT fusion protein can have a different DNA binding and transactivation properties than the wild-type GATA2. GATA2-ERT may not fit easily into mult-protein complexes due to the presence of the extra ERT domain. However in functional assays, GATA2-ERT in presence of Tamoxifen completely blocked CFU-C colony formation an effect similar to the wild-type GATA2. This shows that minor differences in protein structure are tolerated in vivo. It will also be interesting to test if the effects of MSCV-GATA2-ERT vector can be replicated when wild-type GATA2 is driven from a weak promoter or an inducible promoter (Tet-Off systems).

\section{Future Directions}

Using the GATA2-ERT system we presented evidence that GATA2 overexpression confers increased self-renewal of myeloid progenitors and that lower dose of GATA2 is critical for this phenotype. To overcome the drawbacks of the ERT fusion system we need to confirm if this effect can be replicated when wild-type GATA2 is driven from a weak promoter or a tetracycline inducible promoter. Identification of GATA2 targets that mediate the self-renewal effect is important for development of AML therapy and also HSC expansion protocols. The mechanism by which GATA2 regulates Nmyc and HoxA9 potential targets in our study can be addressed by ChIP-Seq experiments on the GATA2-ERT cell lines.

Our studies show that GATA2 overexpression by itself is insufficient to initiate leukemia in transplant and transgenic animals. It will be interesting to test if Flt3-ITD or NPM1 mutations can co-operate with GATA2 overexpression to initiate leukemia. It is unclear how GATA2 is overexpressed in AML. Possible mechanisms could be translocations near GATA2 locus, epigenetic modifications or activation by an upstream regulator of GATA2.

Down regulation of GATA2 is a critical step for the multi-lineage differentiation of HSCs. Our data show that dysregulation of this process can lead to lymphoid block 
and myeloid expansion. Identification of factors that down regulate GATA2 in various lineages is crucial for our understanding of hematopoiesis. In the erythroid lineage the transcription factor GATA1 has been shown to down regulate GATA2 by transcriptional repression. ${ }^{98}$ It will be important to identify the factors that down regulate GATA2 in myeloid and lymphoid lineages. 


\section{LIST OF REFERENCES}

1. Weissman IL. Translating stem and progenitor cell biology to the clinic: barriers and opportunities. Science. 2000;287(5457):1442-1446.

2. Makinodan T. Circulating rat cells in lethally irradiated mice protected with rat bone marrow. Proc Soc Exp Biol Med. 1956;92(1):174-179.

3. Nowell PC, Cole LJ, Habermeyer JG, Roan PL. Growth and continued function of rat marrow cells in X-radiated mice. Cancer Res. 1956;16(3):258-261.

4. Ford CE, Hamerton JL, Barnes DW, Loutit JF. Cytological identification of radiation-chimaeras. Nature. 1956;177(4506):452-454.

5. Till JE, McCulloch EA. A direct measurement of the radiation sensitivity of normal mouse bone marrow cells. Radiat Res. 1961;14:213-222.

6. Becker AJ, McCulloch EA, Till JE. Cytological demonstration of the clonal nature of spleen colonies derived from transplanted mouse marrow cells. Nature. $1963 ; 197: 452-454$.

7. Wu AM, Till JE, Siminovitch L, McCulloch EA. Cytological evidence for a relationship between normal hemotopoietic colony-forming cells and cells of the lymphoid system. $J$ Exp Med. 1968;127(3):455-464.

8. Spangrude GJ, Heimfeld S, Weissman IL. Purification and characterization of mouse hematopoietic stem cells. Science. 1988;241(4861):58-62.

9. Ikuta K, Weissman IL. Evidence that hematopoietic stem cells express mouse ckit but do not depend on steel factor for their generation. Proc Natl Acad Sci U S A. 1992;89(4):1502-1506.

10. Uchida N, Aguila HL, Fleming WH, Jerabek L, Weissman IL. Rapid and sustained hematopoietic recovery in lethally irradiated mice transplanted with purified Thy-1.1lo Lin-Sca-1+ hematopoietic stem cells. Blood. 1994;83(12):3758-3779.

11. Morrison SJ, Weissman IL. The long-term repopulating subset of hematopoietic stem cells is deterministic and isolatable by phenotype. Immunity. 1994;1(8):661673.

12. Morrison SJ, Wandycz AM, Hemmati HD, Wright DE, Weissman IL. Identification of a lineage of multipotent hematopoietic progenitors. Development. 1997;124(10):1929-1939.

13. Reya T, Morrison SJ, Clarke MF, Weissman IL. Stem cells, cancer, and cancer stem cells. Nature. 2001;414(6859):105-111. 
14. Adolfsson J, Mansson R, Buza-Vidas N, et al. Identification of Flt3+ lymphomyeloid stem cells lacking erythro-megakaryocytic potential a revised road map for adult blood lineage commitment. Cell. 2005;121(2):295-306.

15. Bell JJ, Bhandoola A. The earliest thymic progenitors for T cells possess myeloid lineage potential. Nature. 2008;452:764-767.

16. Wada H, Masuda K, Satoh R, et al. Adult T-cell progenitors retain myeloid potential. Nature. 2008;452:768-772.

17. Guo G, Luc S, Marco E, et al. Mapping cellular hierarchy by single-cell analysis of the cell surface repertoire. Cell Stem Cell. 2013;13:492-505.

18. Dzierzak E, Philipsen S. Erythropoiesis: development and differentiation. Cold Spring Harb Perspect Med. 2013;3(4).

19. Orkin SH, Zon LI. Hematopoiesis: an evolving paradigm for stem cell biology. Cell. 2008;132(4):631-644.

20. Muller AM, Medvinsky A, Strouboulis J, Grosveld F, Dzierzak E. Development of hematopoietic stem cell activity in the mouse embryo. Immunity. 1994;1(4):291-301.

21. Inman KE, Downs KM. The murine allantois: emerging paradigms in development of the mammalian umbilical cord and its relation to the fetus. Genesis. 2007;45(5):237-258.

22. Ottersbach K, Dzierzak E. The murine placenta contains hematopoietic stem cells within the vascular labyrinth region. Dev Cell. 2005;8(3):377-387.

23. Gekas C, Dieterlen-Lievre F, Orkin SH, Mikkola HK. The placenta is a niche for hematopoietic stem cells. Dev Cell. 2005;8(3):365-375.

24. Look AT. Oncogenic transcription factors in the human acute leukemias. Science. 1997;278:1059-1064.

25. Kelly LM, Gilliland DG. Genetics of myeloid leukemias. Annu Rev Genomics Hum Genet. 2002;3:179-198.

26. Genomic and epigenomic landscapes of adult de novo acute myeloid leukemia. $N$ Engl J Med. 2013;368:2059-2074.

27. Robb L, Lyons I, Li R, et al. Absence of yolk sac hematopoiesis from mice with a targeted disruption of the scl gene. Proc Natl Acad Sci U S A. 1995;92:7075-7079.

28. Shivdasani RA, Mayer EL, Orkin SH. Absence of blood formation in mice lacking the T-cell leukaemia oncoprotein tal-1/SCL. Nature. 1995;373:432-434. 
29. Porcher C, Swat W, Rockwell K, Fujiwara Y, Alt FW, Orkin SH. The T cell leukemia oncoprotein SCL/tal-1 is essential for development of all hematopoietic lineages. Cell. 1996;86:47-57.

30. Okuda T, van Deursen J, Hiebert SW, Grosveld G, Downing JR. AML1, the target of multiple chromosomal translocations in human leukemia, is essential for normal fetal liver hematopoiesis. Cell. 1996;84:321-330.

31. Mukouyama Y, Chiba N, Hara T, et al. The AML1 transcription factor functions to develop and maintain hematogenic precursor cells in the embryonic aortagonad-mesonephros region. Dev Biol. 2000;220:27-36.

32. North TE, de Bruijn MF, Stacy T, et al. Runx1 expression marks long-term repopulating hematopoietic stem cells in the midgestation mouse embryo. Immunity. 2002;16:661-672.

33. Zhang DE, Zhang P, Wang ND, Hetherington CJ, Darlington GJ, Tenen DG. Absence of granulocyte colony-stimulating factor signaling and neutrophil development in CCAAT enhancer binding protein alpha-deficient mice. Proc Natl Acad Sci U S A. 1997;94(2):569-574.

34. Pevny L, Simon MC, Robertson E, et al. Erythroid differentiation in chimaeric mice blocked by a targeted mutation in the gene for transcription factor GATA-1. Nature. 1991;349(6306):257-260.

35. Mikkola HK, Klintman J, Yang H, et al. Haematopoietic stem cells retain longterm repopulating activity and multipotency in the absence of stem-cell leukaemia SCL/tal-1 gene. Nature. 2003;421(6922):547-551.

36. Ichikawa M, Asai T, Saito T, et al. AML-1 is required for megakaryocytic maturation and lymphocytic differentiation, but not for maintenance of hematopoietic stem cells in adult hematopoiesis. Nat Med. 2004;10(3):299-304.

37. Dzierzak E, Speck NA. Of lineage and legacy: the development of mammalian hematopoietic stem cells. Nat Immunol. 2008;9(2):129-136.

38. Zhang P, Iwasaki-Arai J, Iwasaki H, et al. Enhancement of hematopoietic stem cell repopulating capacity and self-renewal in the absence of the transcription factor C/EBP alpha. Immunity. 2004;21(6):853-863.

39. Rekhtman N, Radparvar F, Evans T, Skoultchi AI. Direct interaction of hematopoietic transcription factors PU.1 and GATA-1: functional antagonism in erythroid cells. Genes Dev. 1999;13(11):1398-1411.

40. Zhang P, Zhang X, Iwama A, et al. PU.1 inhibits GATA-1 function and erythroid differentiation by blocking GATA-1 DNA binding. Blood. 2000;96(8):2641-2648. 
41. Nerlov C, Querfurth E, Kulessa H, Graf T. GATA-1 interacts with the myeloid PU.1 transcription factor and represses PU.1-dependent transcription. Blood. 2000;95(8):2543-2551.

42. Iwasaki H, Mizuno S, Wells RA, Cantor AB, Watanabe S, Akashi K. GATA-1 converts lymphoid and myelomonocytic progenitors into the megakaryocyte/erythrocyte lineages. Immunity. 2003;19(3):451-462.

43. Yamada T, Abe M, Higashi T, et al. Lineage switch induced by overexpression of Ets family transcription factor PU.1 in murine erythroleukemia cells. Blood. 2001;97(8):2300-2307.

44. Galloway JL, Wingert RA, Thisse C, Thisse B, Zon LI. Loss of gatal but not gata2 converts erythropoiesis to myelopoiesis in zebrafish embryos. Dev Cell. 2005;8(1):109-116.

45. Rhodes J, Hagen A, Hsu K, et al. Interplay of pu.1 and gata1 determines myeloerythroid progenitor cell fate in zebrafish. Dev Cell. 2005;8(1):97-108.

46. DeKoter RP, Singh H. Regulation of B lymphocyte and macrophage development by graded expression of PU.1. Science. 2000;288(5470):1439-1441.

47. Kulessa H, Frampton J, Graf T. GATA-1 reprograms avian myelomonocytic cell lines into eosinophils, thromboblasts, and erythroblasts. Genes Dev. 1995;9(10):1250-1262.

48. Wadman IA, Osada H, Grutz GG, et al. The LIM-only protein Lmo2 is a bridging molecule assembling an erythroid, DNA-binding complex which includes the TAL1, E47, GATA-1 and Ldb1/NLI proteins. EMBO J. 1997;16(11):3145-3157.

49. Crispino JD, Lodish MB, MacKay JP, Orkin SH. Use of altered specificity mutants to probe a specific protein-protein interaction in differentiation: the GATA-1:FOG complex. Mol Cell. 1999;3(2):219-228.

50. Hong W, Nakazawa M, Chen YY, et al. FOG-1 recruits the NuRD repressor complex to mediate transcriptional repression by GATA-1. EMBO J. 2005;24(13):2367-2378.

51. Orkin SH. Diversification of haematopoietic stem cells to specific lineages. Nat Rev Genet. 2000;1(1):57-64.

52. Orlic D, Anderson S, Biesecker LG, Sorrentino BP, Bodine DM. Pluripotent hematopoietic stem cells contain high levels of mRNA for c-kit, GATA-2, p45 NF-E2, and c-myb and low levels or no mRNA for c-fms and the receptors for granulocyte colony-stimulating factor and interleukins 5 and 7. Proc Natl Acad Sci U S A. 1995;92(10):4601-4605. 
53. Tsai FY, Orkin SH. Transcription factor GATA-2 is required for proliferation/survival of early hematopoietic cells and mast cell formation, but not for erythroid and myeloid terminal differentiation. Blood. 1997;89(10):36363643.

54. Ito E, Toki T, Ishihara $\mathrm{H}$, et al. Erythroid transcription factor GATA-1 is abundantly transcribed in mouse testis. Nature. 1993;362(6419):466-468.

55. Minegishi N, Ohta J, Yamagiwa H, et al. The mouse GATA-2 gene is expressed in the para-aortic splanchnopleura and aorta-gonads and mesonephros region. Blood. 1999;93(12):4196-4207.

56. Dorfman DM, Wilson DB, Bruns GA, Orkin SH. Human transcription factor GATA-2. Evidence for regulation of preproendothelin-1 gene expression in endothelial cells. J Biol Chem. 1992;267(2):1279-1285.

57. Nardelli J, Thiesson D, Fujiwara Y, Tsai FY, Orkin SH. Expression and genetic interaction of transcription factors GATA-2 and GATA-3 during development of the mouse central nervous system. Dev Biol. 1999;210(2):305-321.

58. Ng YK, George KM, Engel JD, Linzer DI. GATA factor activity is required for the trophoblast-specific transcriptional regulation of the mouse placental lactogen I gene. Development. 1994;120(11):3257-3266.

59. Dasen JS, O'Connell SM, Flynn SE, et al. Reciprocal interactions of Pit1 and GATA2 mediate signaling gradient-induced determination of pituitary cell types. Cell. 1999;97(5):587-598.

60. Kaufman CK, Zhou P, Pasolli HA, et al. GATA-3: an unexpected regulator of cell lineage determination in skin. Genes Dev. 2003;17(17):2108-2122.

61. Kouros-Mehr H, Slorach EM, Sternlicht MD, Werb Z. GATA-3 maintains the differentiation of the luminal cell fate in the mammary gland. Cell. 2006;127(5):1041-1055.

62. Grote D, Boualia SK, Souabni A, et al. Gata3 acts downstream of beta-catenin signaling to prevent ectopic metanephric kidney induction. PLoS Genet. 2008;4(12):e1000316.

63. Molkentin JD. The zinc finger-containing transcription factors GATA-4, -5 , and 6. Ubiquitously expressed regulators of tissue-specific gene expression. $\mathrm{J} \mathrm{Biol}$ Chem. 2000;275(50):38949-38952.

64. Shivdasani RA, Fujiwara Y, McDevitt MA, Orkin SH. A lineage-selective knockout establishes the critical role of transcription factor GATA-1 in megakaryocyte growth and platelet development. EMBO J. 1997;16(13):39653973. 
65. Yu C, Cantor AB, Yang H, et al. Targeted deletion of a high-affinity GATAbinding site in the GATA-1 promoter leads to selective loss of the eosinophil lineage in vivo. $J$ Exp Med. 2002;195(11):1387-1395.

66. Tsai FY, Keller G, Kuo FC, et al. An early haematopoietic defect in mice lacking the transcription factor GATA-2. Nature. 1994;371(6494):221-226.

67. Pandolfi PP, Roth ME, Karis A, et al. Targeted disruption of the GATA3 gene causes severe abnormalities in the nervous system and in fetal liver haematopoiesis. Nat Genet. 1995;11(1):40-44.

68. Ting CN, Olson MC, Barton KP, Leiden JM. Transcription factor GATA-3 is required for development of the T-cell lineage. Nature. 1996;384(6608):474-478.

69. Tsai FY, Browne CP, Orkin SH. Knock-in mutation of transcription factor GATA-3 into the GATA-1 locus: partial rescue of GATA-1 loss of function in erythroid cells. Dev Biol. 1998;196(2):218-227.

70. Takahashi S, Shimizu R, Suwabe N, et al. GATA factor transgenes under GATA1 locus control rescue germline GATA-1 mutant deficiencies. Blood. 2000;96(3):910-916.

71. Ranganath S, Murphy KM. Structure and specificity of GATA proteins in Th2 development. Mol Cell Biol. 2001;21(8):2716-2725.

72. Ko LJ, Engel JD. DNA-binding specificities of the GATA transcription factor family. Mol Cell Biol. 1993;13(7):4011-4022.

73. Merika M, Orkin SH. DNA-binding specificity of GATA family transcription factors. Mol Cell Biol. 1993;13(7):3999-4010.

74. Martin DI, Orkin SH. Transcriptional activation and DNA binding by the erythroid factor GF-1/NF-E1/Eryf 1. Genes Dev. 1990;4(11):1886-1898.

75. Newton A, Mackay J, Crossley M. The N-terminal zinc finger of the erythroid transcription factor GATA-1 binds GATC motifs in DNA. J Biol Chem. 2001;276(38):35794-35801.

76. Fujiwara $\mathrm{T}, \mathrm{O}$ 'Geen $\mathrm{H}$, Keles $\mathrm{S}$, et al. Discovering hematopoietic mechanisms through genome-wide analysis of GATA factor chromatin occupancy. Mol Cell. 2009;36(4):667-681.

77. Wei G, Abraham BJ, Yagi R, et al. Genome-wide analyses of transcription factor GATA3-mediated gene regulation in distinct $\mathrm{T}$ cell types. Immunity. 2011;35(2):299-311. 
78. Yu M, Riva L, Xie H, et al. Insights into GATA-1-mediated gene activation versus repression via genome-wide chromatin occupancy analysis. Mol Cell. 2009;36(4):682-695.

79. Chen Y, Bates DL, Dey R, et al. DNA binding by GATA transcription factor suggests mechanisms of DNA looping and long-range gene regulation. Cell Rep. 2012;2(5):1197-1206.

80. Minegishi N, Ohta J, Suwabe N, et al. Alternative promoters regulate transcription of the mouse GATA-2 gene. J Biol Chem. 1998;273(6):3625-3634.

81. Zhou Y, Lim KC, Onodera K, et al. Rescue of the embryonic lethal hematopoietic defect reveals a critical role for GATA-2 in urogenital development. EMBOJ. 1998;17(22):6689-6700.

82. Khandekar M, Suzuki N, Lewton J, Yamamoto M, Engel JD. Multiple, distant Gata2 enhancers specify temporally and tissue-specific patterning in the developing urogenital system. Mol Cell Biol. 2004;24(23):10263-10276.

83. Zhou Y, Yamamoto M, Engel JD. GATA2 is required for the generation of V2 interneurons. Development. 2000;127(17):3829-3838.

84. Khandekar M, Brandt W, Zhou Y, et al. A Gata2 intronic enhancer confers its pan-endothelia-specific regulation. Development. 2007;134(9):1703-1712.

85. Lim KC, Hosoya T, Brandt W, et al. Conditional Gata2 inactivation results in HSC loss and lymphatic mispatterning. J Clin Invest. 2012;122(10):3705-3717.

86. Martowicz ML, Grass JA, Boyer ME, Guend H, Bresnick EH. Dynamic GATA factor interplay at a multicomponent regulatory region of the GATA-2 locus. $J$ Biol Chem. 2005;280(3):1724-1732.

87. Grass JA, Jing H, Kim SI, et al. Distinct functions of dispersed GATA factor complexes at an endogenous gene locus. Mol Cell Biol. 2006;26(19):7056-7067.

88. Wozniak RJ, Boyer ME, Grass JA, Lee Y, Bresnick EH. Context-dependent GATA factor function: combinatorial requirements for transcriptional control in hematopoietic and endothelial cells. J Biol Chem. 2007;282(19):14665-14674.

89. Snow JW, Trowbridge JJ, Fujiwara T, et al. A single cis element maintains repression of the key developmental regulator Gata2. PLoS Genet. 2010;6(9).

90. Snow JW, Trowbridge JJ, Johnson KD, et al. Context-dependent function of "GATA switch" sites in vivo. Blood. 2011;117(18):4769-4772.

91. Rodrigues NP, Janzen V, Forkert R, et al. Haploinsufficiency of GATA-2 perturbs adult hematopoietic stem-cell homeostasis. Blood. 2005;106(2):477-484. 
92. Ling KW, Ottersbach K, van Hamburg JP, et al. GATA-2 plays two functionally distinct roles during the ontogeny of hematopoietic stem cells. J Exp Med. 2004;200(7):871-882.

93. Moignard V, Macaulay IC, Swiers G, et al. Characterization of transcriptional networks in blood stem and progenitor cells using high-throughput single-cell gene expression analysis. Nat Cell Biol. 2013.

94. Watkins NA, Gusnanto A, de Bono B, et al. A HaemAtlas: characterizing gene expression in differentiated human blood cells. Blood. 2009;113(19):e1-9.

95. Persons DA, Allay JA, Allay ER, et al. Enforced expression of the GATA-2 transcription factor blocks normal hematopoiesis. Blood. 1999;93(2):488-499.

96. Tipping AJ, Pina C, Castor A, et al. High GATA-2 expression inhibits human hematopoietic stem and progenitor cell function by effects on cell cycle. Blood. 2009;113(12):2661-2672.

97. Weiss MJ, Yu C, Orkin SH. Erythroid-cell-specific properties of transcription factor GATA-1 revealed by phenotypic rescue of a gene-targeted cell line. $\mathrm{Mol}$ Cell Biol. 1997;17(3):1642-1651.

98. Grass JA, Boyer ME, Pal S, Wu J, Weiss MJ, Bresnick EH. GATA-1-dependent transcriptional repression of GATA-2 via disruption of positive autoregulation and domain-wide chromatin remodeling. Proc Natl Acad Sci U S A. 2003;100(15):8811-8816.

99. Walsh JC, DeKoter RP, Lee HJ, et al. Cooperative and antagonistic interplay between PU.1 and GATA-2 in the specification of myeloid cell fates. Immunity. 2002;17(5):665-676.

100. Chou ST, Khandros E, Bailey LC, et al. Graded repression of PU.1/Sfpi1 gene transcription by GATA factors regulates hematopoietic cell fate. Blood. 2009;114(5):983-994.

101. Jippo T, Mizuno H, Xu Z, Nomura S, Yamamoto M, Kitamura Y. Abundant expression of transcription factor GATA-2 in proliferating but not in differentiated mast cells in tissues of mice: demonstration by in situ hybridization. Blood. 1996;87(3):993-998.

102. Iwasaki H, Mizuno S, Arinobu Y, et al. The order of expression of transcription factors directs hierarchical specification of hematopoietic lineages. Genes Dev. 2006;20(21):3010-3021.

103. Wechsler J, Greene M, McDevitt MA, et al. Acquired mutations in GATA1 in the megakaryoblastic leukemia of Down syndrome. Nat Genet. 2002;32:148-152. 
104. Huang Z, Dore LC, Li Z, et al. GATA-2 reinforces megakaryocyte development in the absence of GATA-1. Mol Cell Biol. 2009;29(18):5168-5180.

105. Li Z, Godinho FJ, Klusmann JH, Garriga-Canut M, Yu C, Orkin SH. Developmental stage-selective effect of somatically mutated leukemogenic transcription factor GATA1. Nat Genet. 2005;37:613-619.

106. Ezoe S, Matsumura I, Nakata S, et al. GATA-2/estrogen receptor chimera regulates cytokine-dependent growth of hematopoietic cells through accumulation of p21(WAF1) and p27(Kip1) proteins. Blood. 2002;100(10):3512-3520.

107. Wilson NK, Foster SD, Wang X, et al. Combinatorial transcriptional control in blood stem/progenitor cells: genome-wide analysis of ten major transcriptional regulators. Cell Stem Cell. 2010;7(4):532-544.

108. Li L, Jothi R, Cui K, et al. Nuclear adaptor Ldb1 regulates a transcriptional program essential for the maintenance of hematopoietic stem cells. Nat Immunol. 2011;12(2):129-136.

109. Stachura DL, Chou ST, Weiss MJ. Early block to erythromegakaryocytic development conferred by loss of transcription factor GATA-1. Blood. 2006;107(1):87-97.

110. Bresnick EH, Katsumura KR, Lee HY, Johnson KD, Perkins AS. Master regulatory GATA transcription factors: mechanistic principles and emerging links to hematologic malignancies. Nucleic Acids Res. 2012;40(13):5819-5831.

111. Jing H, Vakoc CR, Ying L, et al. Exchange of GATA factors mediates transitions in looped chromatin organization at a developmentally regulated gene locus. Mol Cell. 2008;29(2):232-242.

112. Tripic T, Deng W, Cheng Y, et al. SCL and associated proteins distinguish active from repressive GATA transcription factor complexes. Blood. 2009;113(10):2191-2201.

113. Dore LC, Amigo JD, Dos Santos CO, et al. A GATA-1-regulated microRNA locus essential for erythropoiesis. Proc Natl Acad Sci U S A. 2008;105(9):33333338 .

114. Im H, Grass JA, Johnson KD, et al. Chromatin domain activation via GATA-1 utilization of a small subset of dispersed GATA motifs within a broad chromosomal region. Proc Natl Acad Sci U S A. 2005;102(47):17065-17070.

115. Gregory GD, Miccio A, Bersenev A, et al. FOG1 requires NuRD to promote hematopoiesis and maintain lineage fidelity within the megakaryocytic-erythroid compartment. Blood. 2010;115(11):2156-2166. 
116. Dore LC, Chlon TM, Brown CD, White KP, Crispino JD. Chromatin occupancy analysis reveals genome-wide GATA factor switching during hematopoiesis. Blood. 2012;119(16):3724-3733.

117. Wozniak RJ, Keles S, Lugus JJ, et al. Molecular hallmarks of endogenous chromatin complexes containing master regulators of hematopoiesis. Mol Cell Biol. 2008;28(21):6681-6694.

118. Hahn CN, Chong CE, Carmichael CL, et al. Heritable GATA2 mutations associated with familial myelodysplastic syndrome and acute myeloid leukemia. Nat Genet. 2011;43(10):1012-1017.

119. Ostergaard P, Simpson MA, Connell FC, et al. Mutations in GATA2 cause primary lymphedema associated with a predisposition to acute myeloid leukemia (Emberger syndrome). Nat Genet. 2011;43(10):929-931.

120. Hsu AP, Sampaio EP, Khan J, et al. Mutations in GATA2 are associated with the autosomal dominant and sporadic monocytopenia and mycobacterial infection (MonoMAC) syndrome. Blood. 2011;118(10):2653-2655.

121. Dickinson RE, Griffin H, Bigley V, et al. Exome sequencing identifies GATA-2 mutation as the cause of dendritic cell, monocyte, B and NK lymphoid deficiency. Blood. 2011;118(10):2656-2658.

122. Zhang SJ, Ma LY, Huang QH, et al. Gain-of-function mutation of GATA-2 in acute myeloid transformation of chronic myeloid leukemia. Proc Natl Acad Sci U $S$ A. 2008;105(6):2076-2081.

123. Gruber TA, Larson Gedman A, Zhang J, et al. An Inv(16)(p13.3q24.3)-encoded CBFA2T3-GLIS2 fusion protein defines an aggressive subtype of pediatric acute megakaryoblastic leukemia. Cancer Cell. 2012;22(5):683-697.

124. Feil R, Wagner J, Metzger D, Chambon P. Regulation of Cre recombinase activity by mutated estrogen receptor ligand-binding domains. Biochem Biophys Res Commun. 1997;237(3):752-757.

125. Hanawa H, Kelly PF, Nathwani AC, et al. Comparison of various envelope proteins for their ability to pseudotype lentiviral vectors and transduce primitive hematopoietic cells from human blood. Mol Ther. 2002;5(3):242-251.

126. Dickins RA, Hemann MT, Zilfou JT, et al. Probing tumor phenotypes using stable and regulated synthetic microRNA precursors. Nat Genet. 2005;37(11):12891295.

127. Hanawa H, Hematti P, Keyvanfar K, et al. Efficient gene transfer into rhesus repopulating hematopoietic stem cells using a simian immunodeficiency virusbased lentiviral vector system. Blood. 2004;103(11):4062-4069. 
128. Paddison PJ, Cleary M, Silva JM, et al. Cloning of short hairpin RNAs for gene knockdown in mammalian cells. Nat Methods. 2004;1(2):163-167.

129. Kiel MJ, Yilmaz OH, Iwashita T, Terhorst C, Morrison SJ. SLAM family receptors distinguish hematopoietic stem and progenitor cells and reveal endothelial niches for stem cells. Cell. 2005;121(7):1109-1121.

130. Akashi K, Traver D, Miyamoto T, Weissman IL. A clonogenic common myeloid progenitor that gives rise to all myeloid lineages. Nature. 2000;404(6774):193197.

131. Kondo M, Weissman IL, Akashi K. Identification of clonogenic common lymphoid progenitors in mouse bone marrow. Cell. 1997;91(5):661-672.

132. Du Y, Jenkins NA, Copeland NG. Insertional mutagenesis identifies genes that promote the immortalization of primary bone marrow progenitor cells. Blood. 2005;106(12):3932-3939.

133. Eischen CM, Woo D, Roussel MF, Cleveland JL. Apoptosis triggered by Mycinduced suppression of $\mathrm{Bcl}-\mathrm{X}(\mathrm{L})$ or $\mathrm{Bcl}-2$ is bypassed during lymphomagenesis. Mol Cell Biol. 2001;21(15):5063-5070.

134. Haldar M, Hedberg ML, Hockin MF, Capecchi MR. A CreER-based random induction strategy for modeling translocation-associated sarcomas in mice. Cancer Res. 2009;69(8):3657-3664.

135. Lurie LJ, Boyer ME, Grass JA, Bresnick EH. Differential GATA factor stabilities: implications for chromatin occupancy by structurally similar transcription factors. Biochemistry. 2008;47(3):859-869.

136. Hung HL, Lau J, Kim AY, Weiss MJ, Blobel GA. CREB-Binding protein acetylates hematopoietic transcription factor GATA-1 at functionally important sites. Mol Cell Biol. 1999;19(5):3496-3505.

137. Rosenbauer F, Koschmieder S, Steidl U, Tenen DG. Effect of transcription-factor concentrations on leukemic stem cells. Blood. 2005;106(5):1519-1524.

138. Cheshier SH, Morrison SJ, Liao X, Weissman IL. In vivo proliferation and cell cycle kinetics of long-term self-renewing hematopoietic stem cells. Proc Natl Acad Sci U S A. 1999;96(6):3120-3125.

139. Bradford GB, Williams B, Rossi R, Bertoncello I. Quiescence, cycling, and turnover in the primitive hematopoietic stem cell compartment. Exp Hematol. 1997;25(5):445-453.

140. Wilson A, Laurenti E, Oser G, et al. Hematopoietic stem cells reversibly switch from dormancy to self-renewal during homeostasis and repair. Cell. 2008;135(6):1118-1129. 
141. Steinman RA. Cell cycle regulators and hematopoiesis. Oncogene. 2002;21(21):3403-3413.

142. Heyworth C, Gale K, Dexter M, May G, Enver T. A GATA-2/estrogen receptor chimera functions as a ligand-dependent negative regulator of self-renewal. Genes Dev. 1999;13(14):1847-1860.

143. Venezia TA, Merchant AA, Ramos CA, et al. Molecular signatures of proliferation and quiescence in hematopoietic stem cells. PLoS Biol. 2004;2(10):e301.

144. Rodrigues NP, Boyd AS, Fugazza C, et al. GATA-2 regulates granulocytemacrophage progenitor cell function. Blood. 2008;112(13):4862-4873.

145. Kawagoe H, Kandilci A, Kranenburg TA, Grosveld GC. Overexpression of NMyc rapidly causes acute myeloid leukemia in mice. Cancer Res. 2007;67(22):10677-10685.

146. Armstrong SA, Staunton JE, Silverman LB, et al. MLL translocations specify a distinct gene expression profile that distinguishes a unique leukemia. Nat Genet. 2001;30:41-47.

147. Ayton PM, Cleary ML. Transformation of myeloid progenitors by MLL oncoproteins is dependent on Hoxa7 and Hoxa9. Genes Dev. 2003;17:2298-2307.

148. Thorsteinsdottir U, Mamo A, Kroon E, et al. Overexpression of the myeloid leukemia-associated Hoxa9 gene in bone marrow cells induces stem cell expansion. Blood. 2002;99(1):121-129.

149. Calvo KR, Sykes DB, Pasillas M, Kamps MP. Hoxa9 immortalizes a granulocytemacrophage colony-stimulating factor-dependent promyelocyte capable of biphenotypic differentiation to neutrophils or macrophages, independent of enforced meis expression. Mol Cell Biol. 2000;20(9):3274-3285.

150. Miyamoto T, Iwasaki H, Reizis B, et al. Myeloid or lymphoid promiscuity as a critical step in hematopoietic lineage commitment. Dev Cell. 2002;3(1):137-147.

151. Akashi K, He X, Chen J, et al. Transcriptional accessibility for genes of multiple tissues and hematopoietic lineages is hierarchically controlled during early hematopoiesis. Blood. 2003;101(2):383-389.

152. Mansson R, Hultquist A, Luc S, et al. Molecular evidence for hierarchical transcriptional lineage priming in fetal and adult stem cells and multipotent progenitors. Immunity. 2007;26(4):407-419.

153. Ng SY, Yoshida T, Zhang J, Georgopoulos K. Genome-wide lineage-specific transcriptional networks underscore Ikaros-dependent lymphoid priming in hematopoietic stem cells. Immunity. 2009;30(4):493-507. 
154. Sridharan R, Smale ST. Predominant interaction of both Ikaros and Helios with the NuRD complex in immature thymocytes. $J$ Biol Chem. 2007;282(41):3022730238 .

155. Bottardi S, Mavoungou L, Bourgoin V, Mashtalir N, Affar EB, Milot E. Direct Protein Interactions Are Responsible for Ikaros-Gata and Ikaros-Cdk9 Cooperativity in Hematopoietic Cells. Mol Cell Biol. 2013.

156. Stehling-Sun S, Dade J, Nutt SL, DeKoter RP, Camargo FD. Regulation of lymphoid versus myeloid fate 'choice' by the transcription factor Mef2c. Nat Immunol. 2009;10(3):289-296.

157. Dias S, Mansson R, Gurbuxani S, Sigvardsson M, Kee BL. E2A proteins promote development of lymphoid-primed multipotent progenitors. Immunity. 2008;29(2):217-227.

158. Vicente C, Vazquez I, Conchillo A, et al. Overexpression of GATA2 predicts an adverse prognosis for patients with acute myeloid leukemia and it is associated with distinct molecular abnormalities. Leukemia. 2012;26(3):550-554.

159. Luesink M, Hollink IH, van der Velden VH, et al. High GATA2 expression is a poor prognostic marker in pediatric acute myeloid leukemia. Blood. 2012;120(10):2064-2075.

160. Cozzio A, Passegue E, Ayton PM, Karsunky H, Cleary ML, Weissman IL. Similar MLL-associated leukemias arising from self-renewing stem cells and short-lived myeloid progenitors. Genes Dev. 2003;17(24):3029-3035.

161. Krivtsov AV, Twomey D, Feng Z, et al. Transformation from committed progenitor to leukaemia stem cell initiated by MLL-AF9. Nature. 2006;442(7104):818-822.

162. Chen W, Kumar AR, Hudson WA, et al. Malignant transformation initiated by Mll-AF9: gene dosage and critical target cells. Cancer Cell. 2008;13(5):432-440.

163. Huntly BJ, Shigematsu H, Deguchi K, et al. MOZ-TIF2, but not BCR-ABL, confers properties of leukemic stem cells to committed murine hematopoietic progenitors. Cancer Cell. 2004;6(6):587-596.

164. Calvo KR, Sykes DB, Pasillas MP, Kamps MP. Nup98-HoxA9 immortalizes myeloid progenitors, enforces expression of Hoxa9, Hoxa7 and Meis1, and alters cytokine-specific responses in a manner similar to that induced by retroviral coexpression of Hoxa9 and Meis1. Oncogene. 2002;21(27):4247-4256.

165. Kumar MS, Hancock DC, Molina-Arcas M, et al. The GATA2 Transcriptional Network Is Requisite for RAS Oncogene-Driven Non-Small Cell Lung Cancer. Cell. 2012;149(3):642-655. 
166. Bigley V, Haniffa M, Doulatov S, et al. The human syndrome of dendritic cell, monocyte, B and NK lymphoid deficiency. $J$ Exp Med. 2011;208(2):227-234.

167. Vinh DC, Patel SY, Uzel G, et al. Autosomal dominant and sporadic monocytopenia with susceptibility to mycobacteria, fungi, papillomaviruses, and myelodysplasia. Blood. 2010;115(8):1519-1529. 


\section{VITA}

Satish Kumar Nandakumar was born in Chennai, India in 1980. He completed the Bachelor of Medicine, Bachelor of Surgery (M.B.,B.S.) degree from Madras Medical College in June 2004. In 2006, he enrolled in the PhD program in the University of Tennessee Health Science Center. In 2007, he joined Dr. Derek Persons Lab at the St. Jude Children's Research Hospital. He presented his work in the American Society of Hematology (ASH) Annual Meeting in 2012. He is expected to graduate in May 2014. 